



\title{
Dialogische Gesprächsführung im Unterricht
}

\section{Interventionsansatz, Instrumente und Videokodierungen}

\author{
herausgegeben von \\ Maralena Weil, Alexander Gröschner, \\ Ann-Kathrin Schindler, Ricardo Böheim, \\ Dennis Hauk \& Tina Seidel
}

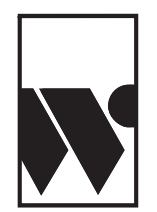

Waxmann 2020

Münster • New York 
Bibliografische Informationen der Deutschen Nationalbibliothek Die Deutsche Nationalbibliothek verzeichnet diese Publikation in der Deutschen Nationalbibliografie; detaillierte bibliografische Daten sind im Internet über http://dnb.dnb.de abrufbar.

Creative Commons-Lizenz Namensnennung - Nicht kommerziell Keine Bearbeitung CC BY-NC ND 3.0 Deutschland

\section{(c) (1) $\Theta \Theta$ \\ BY NC ND}

Print-ISBN 978-3-8309-4160-6

E-Book-ISBN 978-3-8309-9160-1

doi https://10.31244/9783830991601

(C) Waxmann Verlag $\mathrm{GmbH}, 2020$

Steinfurter Straße 555, 48159 Münster

www.waxmann.com

info@waxmann.com

Umschlaggestaltung: Anne Breitenbach, Münster Umschlagfoto: (c) TUM Astrid Eckert 


\section{Inhalt}

Alexander Gröschner, Maralena Weil, Ann-Kathrin Schindler,

Ricardo Böheim, Tina Seidel

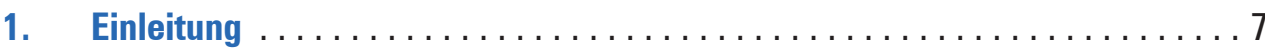

Ricardo Böheim, Alexander Gröschner, Maralena Weil,

Ann-Kathrin Schindler \& Tina Seidel

2. Konzeption der "Dialogue II“-Studie und Erhebungsablauf . . . . . . . 11

2.1 Konzeption und Vorstellung der Fortbildungsangebote $\ldots \ldots \ldots \ldots \ldots \ldots 11$

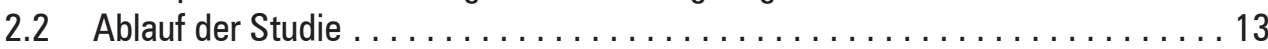

2.3 Richtlinien zur Videoaufzeichnungen in „Dialogue $\|^{\prime \prime} \ldots \ldots \ldots \ldots \ldots \ldots \ldots \ldots \ldots$

2.4 Technische Ausrüstung: Verwendete Hard- und Software in „Dialogue II“ . . . . 16

Ann-Kathrin Schindler, Ricardo Böheim, Maralena Weil,

Alexander Gröschner \& Tina Seidel

3. Videoinstrument „Dialogische Unterrichtsgesprächsführung“ -

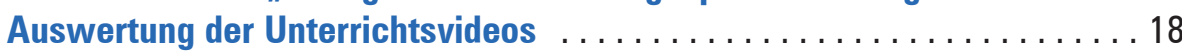

3.1 Prämissen zur Entwicklung des Instruments $\ldots \ldots \ldots \ldots \ldots \ldots \ldots \ldots \ldots$

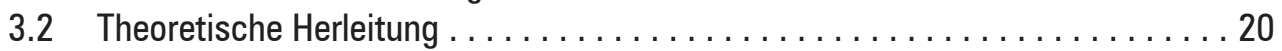

3.3 Kodierungsverfahren und Skalen $\ldots \ldots \ldots \ldots \ldots \ldots \ldots \ldots \ldots \ldots \ldots \ldots$

Alexander Gröschner, Dennis Hauk, Martina Alles, Richard Klöden,

Ann-Kathrin Schindler, Maralena Weil \& Tina Seidel

4. Gesprächs- und Lernkultur im evidenzbasierten Fortbildungsprogramm -

Auswertung der videographierten Veranstaltungen der Fortbildungen . . . . 53

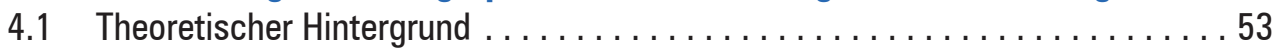

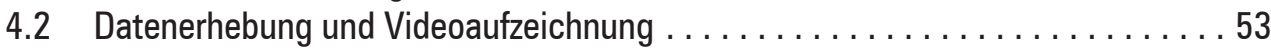

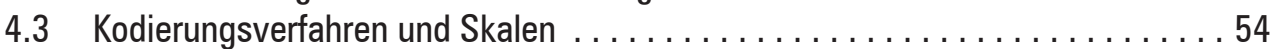

Alexander Gröschner, Ann-Kathrin Schindler, Maralena Weil,

Ricardo Böheim \& Tina Seidel

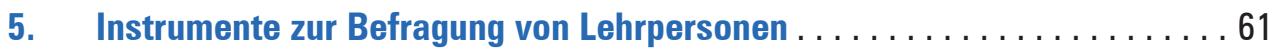

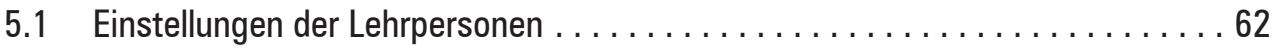

5.2 Befragung der Lehrenden über deren situationale Wahrnehmung der

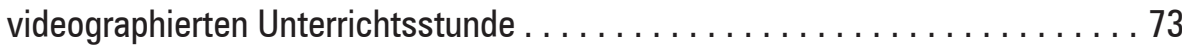

5.3 "Track your Fortbildung" - Online Tool zur Prozessbegleitung der Fortbildung . . 74

Alexander Gröschner, Ann-Kathrin Schindler, Maralena Weil, Ricardo Böheim, Maximilian Knogler \& Tina Seidel

6. Instrumente zur Befragung von Lernenden. ................ 75

6.1 Befragung der Lernenden über deren überdauernden Merkmale . . . . . . . 76

6.2 Befragung der Lernenden über deren situationale Wahrnehmung $\ldots \ldots \ldots \ldots 78$

7. Publikationen im Projekt Dialogue $\ldots \ldots \ldots \ldots \ldots \ldots \ldots \ldots \ldots \ldots \ldots$

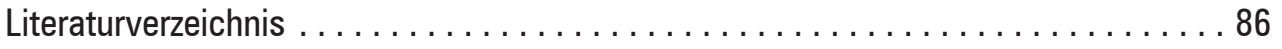





\section{Alexander Gröschner, Maralena Weil, Ann-Kathrin Schindler, Ricardo Böheim, Tina Seidel}

\section{Einleitung}

Die Implementation von dialogischen Ansätzen in die Gestaltung von Unterricht rückt immer stärker in den Fokus von Lehrerausbildung sowie Fort- und Weiterbildung. Ein Grund sind die zunehmenden Forschungsergebnisse, die die Lernwirksamkeit dialogisch orientierter Lehrer/innen-Schüler/innen-Interaktionen wiederholt nachgewiesen haben (Kiemer, Gröschner, Pehmer, \& Seidel, 2015; Mercer \& Littleton, 2007; Osborne, Borko, Fishman, Gomez Zaccarelli, Berson, Busch, Reigh, \& Tseng, 2019). Vor allem die Unterrichtskommunikationsforschung fokussiert die Identifikation von Elementen des lernwirksamen Unterrichtsgesprächs und beleuchtet, wie diese von Lehrpersonen in ihre Unterrichtspraxis implementiert werden können (Howe \& Abedin, 2013; Lipowsky et al., 2009; Mercer \& Dawes, 2014; Mortimer \& Scott, 2010; Seidel, 2014; Seidel \& Prenzel, 2006). Das Thema ist von hoher Relevanz, da Studien in verschiedenen Schulfächern im Primar- und Sekundarbereich, mit qualitativen und/oder quantitativen Forschungsansätzen, zeigen, dass der verbale Austausch zwischen Lehrperson und Schülerinnen und Schülern häufig eng geführte, von der Lehrperson dominierte Kommunikationsstrukturen aufweist (Gillies, 2014; Jurik, Gröschner, \& Seidel, 2013; Osborne, Simon, Christodoulou, Howell-Richardson, \& Richardson, 2013). Die Unterrichtskommunikation weist demzufolge stark autoritär interpretierte instruktionale Routinen des Lehrpersonenhandelns auf, die auch als das „I-R-EMuster" (Mehan, 1979; Sinclair \& Coulthard, 1975), bestehend aus Instruktion (I)Antwort (R)-Evaluation (E), bezeichnet werden. Dieses Muster, welches im Grunde ein Skript für kommunikative Handlungen und Verhaltensweisen von Lehrperson und Schülerinnen und Schülern darstellt, schlägt sich häufig in negativen Lernergebnissen der Schülerinnen und Schüler sowie einer niedrigen Motivation nieder (Resnick, Asterhan, \& Clarke, 2015).

Aus den aufgezeigten Gründen wurden vor allem im englischsprachigen Raum theoretische Ansätze des "dialogic teaching“ (Alexander, 2018) entwickelt, die auf eine Förderung der verbalen Lehrer/in-Schüler/innen-Interaktion abzielen. Alexander (Alexander, 2018) charakterisiert „dialogische Unterrichtsgesprächsführung“ wie folgt:

Hence, the effort, to which all interested in dialogic pedagogy subscribe, to move beyond the monologic dominance of recitation/IRE and develop patterns of classroom interaction that open up students' speaking and listening, and hence their thinking, and which strive to distribute the ownership of talk more equitably. (...) [It] devotes equal attention to the quality of teacher and student talk, and to the agency of others - fellow students as well as teachers - in the latter. It also rejects the view that there is one right way to maximise talk's quality and power (...), and instead advances the need for every teacher to develop a broad repertoire of talk-based pedagogical skills and strategies and to draw on these to expand and refine the talk repertoires and capacities of their students. (Alexander, 2018, S. 563)

In aktuellen empirischen Studien zur "dialogischen Unterrichtsgesprächsführung“ lassen sich vor allem Ansätze aufzeigen, die das notwendige Repertoire der Lehrpersonen in einer erhöhten Schülerbeteiligung im Kontext einer besseren Qualität der Lehrer/in-Schüler/innen-Kommunikation betonen (Pielmeier et al., 2018; 
Resnick et al., 2015; Sedova et al., 2019; Walshaw \& Anthony, 2008). Aus überfachlicher Sicht werden hierbei wirksame Gesprächsstrategien („talk moves") und Methoden („talk activities") aufgezeigt (Gröschner, Schindler, Holzberger, Alles, \& Seidel, 2018; O'Connor, Michaels, Chapin, \& Harbaugh, 2017), die durch eine veränderte Unterrichtsgestaltung das Lernen der Schülerinnen und Schüler fördern. Strategien seitens der Lehrperson sind insbesondere verbale Ansätze wie z.B. Elaborationsfragen, prozessorientierte Rückmeldungen (Pehmer, Gröschner, \& Seidel, 2015a) sowie Regeln und die Einhaltung sozial erwünschter Normen der Kommunikation (Hofmann \& Ruthven, 2018) während des Unterrichts. Diskursfördernde Unterrichtsmethoden stellen vor allem Gestaltungsmerkmale der Lernumgebung und Unterrichtsmethoden (z.B. Meldekette, Gruppenpuzzle) dar, welche den interaktiven Austausch und die verstärkte Schülerbeteiligung adressieren (Michaels \& O'Connor, 2012). Programme wie "Accountable Talk" (Michaels, O'Connor, \& Resnick, 2008; O'Connor et al., 2017), „CamTalk“ (Higham, Brindley, \& van de Pol, 2014), „Dialogic Video Cycle“ (Gröschner, Seidel, Kiemer, \& Pehmer, 2015) oder "Socrates 2.0" (Zimmermann, Moser, Wischgoll, Reusser, \& Pauli, 2019) unterstützen Lehrpersonen darin, ihren pädagogischen Herausforderungen im Rahmen der Unterrichtskommunikation professionell zu begegnen (Resnick et al., 2015). Die Angebote orientieren sich in der Planung, Durchführung und Reflexion der Maßnahmen - mehr oder weniger explizit - an Merkmalen effektiver Fort- und Weiterbildung, die aus der Forschung bekannt sind (DarlingHammond, Hyler, \& Gardner, 2017; Desimone, 2009; Kennedy, 2016; Wilson, 2013). $\mathrm{Zu}$ den Merkmalen gehören Lernaktivitäten innerhalb der Fortbildung, die einen unmittelbaren Bezug zur beruflichen Praxis der Lehrpersonen herstellen, kohärent zu den Lehrplänen sind, Rahmenbedingungen im schulischen Kontext berücksichtigen, den Erfahrungsaustausch der Lehrpersonen untereinander ermöglichen und mehrmalige Veranstaltungen/Workshops über einen längeren Zeitraum umfassen (Desimone, 2009). Zudem wird in letzter Zeit auch immer mehr die Rolle der Fortbildungsleitung stärker berücksichtigt (Alles, Seidel, \& Gröschner, 2018; Gröschner, Seidel, Pehmer, \& Kiemer, 2014), die in moderierender Funktion („Facilitation“) (neues) Fachwissen und pädagogisches Wissen einbringt und das Lernen der Lehrpersonen unterstützt.

Ziel des vorliegenden Bandes ist, anhand des DFG-Projekts „Dialogue II: Lernen von Lehrpersonen am Beispiel des Klassengesprächs - Eine videobasierte Interventionsstudie " ${ }^{1}$ Erhebungsinstrumente und Kodiermanuale vorzustellen, die zur wissenschaftlichen Begleitung der Interventionsstudie entwickelt und eingesetzt wurden. Um möglichst nah am Fortbildungskontext $\mathrm{zu}$ sein, wurden neben dem Rückgriff auf bestehende Instrumente auch teilweise neue Erhebungs- und Analyseansätze entwickelt. Der Bedarf der Neuentwicklung entstand, da trotz der gestiegenen Aufmerksamkeit des Themas „dialogische Unterrichtskommunikation“ derzeit nur wenige erprobte Befragungs- und Kodierinstrumente vorliegen. Zudem steht in der Forschungslandschaft im Bereich von Fortbildungen immer die Frage nach möglichst proximalen Instrumenten (Seidel \& Shavelson, 2007) im Raum. Aus dem Grund ist der vorliegende Band auch als Einladung an die scientific community zu verstehen, die im Projekt entstandenen Fragebögen und Kodierleitfäden in eigenen Studien einzusetzen und zu adaptieren.

Die entwickelten und eingesetzten Erhebungsinstrumente können entlang des internationalen Modells von Clarke und Hollingsworth (Clarke \& Hollingsworth, 2002) verschiedenen Wirkungsbereichen zugeordnet werden, in denen Veränderungen durch die Intervention zu erwarten sind.

1 DFG-Förderkennzeichen SE1397/5-2 und GR3432/4-3 und Kultusministerium X.7-BO4106/ $240 / 23$ 


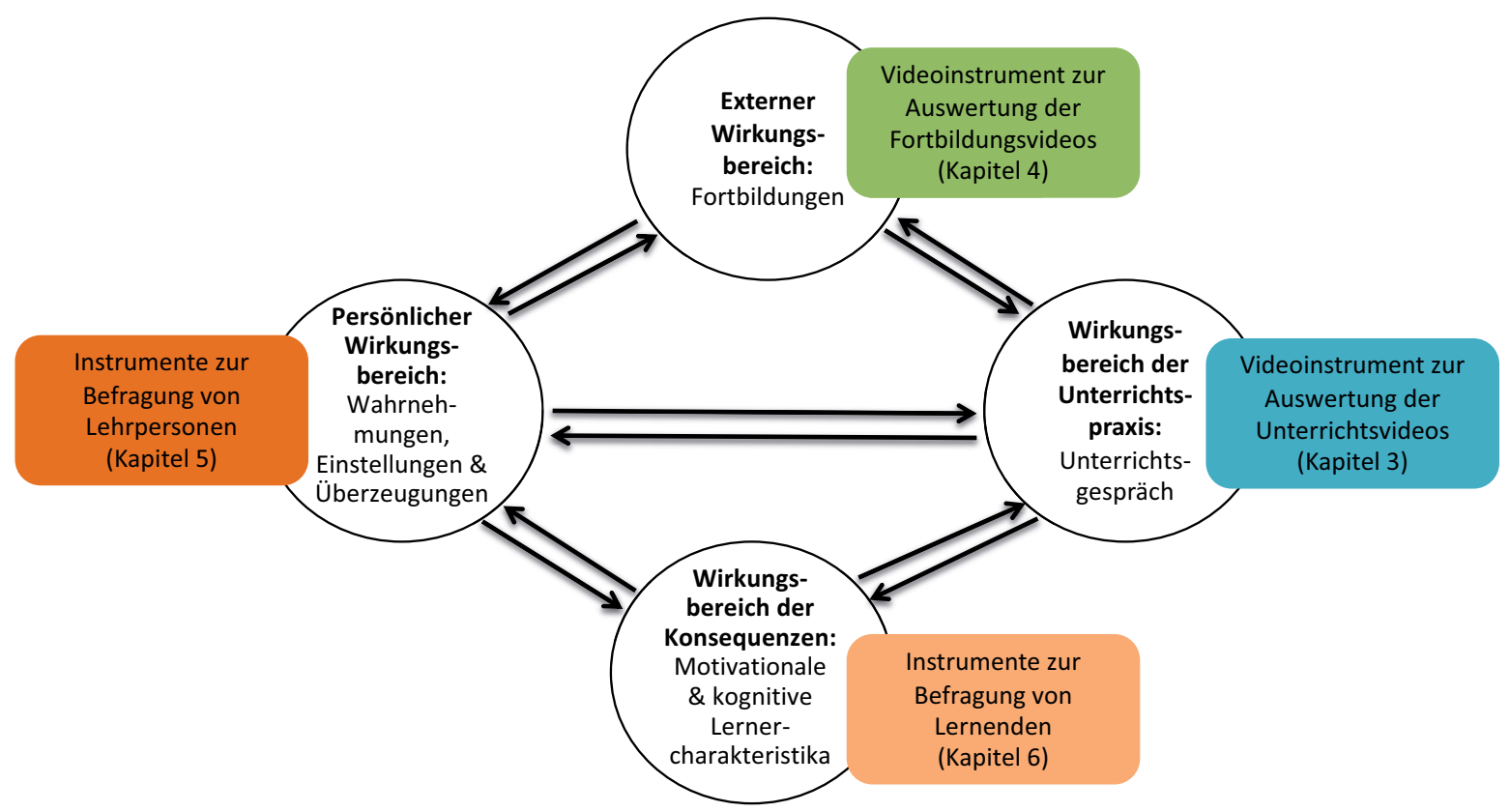

Abbildung 1.1: Einordnung der Erhebungsinstrumente in das adaptierte Modell von Clarke \& Hollingsworth (2002)

Der externe Wirkungsbereich bildet das Fortbildungsprogramm selbst ab, welches Einfluss auf die Unterrichtspraxis (das Unterrichtsgespräch - Wirkungsbereich der Unterrichtspraxis), die Lehrperson (deren Wahrnehmungen, Einstellungen und Überzeugungen - persönlicher Wirkungsbereich) sowie die Schülerinnen und Schüler (z.B. hinsichtlich motivational-affektiver Aspekte - Wirkungsbereich der Konsequenzen) nehmen kann. Die vorgestellten Wirkungsbereiche stellen den Advanced Organizer dieses Bandes dar.

Nach einer detaillierten Darstellung der Dialogue-II-Studie sowie technischen Details zur Anfertigung der Videographien in Kapitel 2, nehmen sich Kapitel 3 und Kapitel 4 den in der Studie eingesetzten Videoinstrumenten an.

Das in Kapitel 3 vorgestellte Auswertungsinstrument für die Unterrichtsvideographien wurde entlang aktueller internationaler Auswertungsinstrumente (u.a. Reznitskaya, Wilkinson, \& Oyler, 2016) entwickelt. Neben der gezielten Entwicklung für die Dialogue-II-Studie, soll dieses als ein ökonomisches Instrument zur Erfassung dialogischer Unterrichtsgesprächsführung auch einen Beitrag in der aktuellen Unterrichtsforschung im deutschsprachigen Raum leisten.

Im Kapitel 4 wird das für den Projektkontext entwickelte Auswertungsinstrument für die ebenfalls videographierten Fortbildungsworkshops vorgestellt. Zentrales Anliegen des Projekts war es, detaillierte Einblicke in die Lernprozesse der Lehrpersonen während der Fortbildung zu erhalten sowie sicher zu stellen, dass die Fortbildungsworkshops evidenzbasiert gängigen Qualitätskriterien (u.a. Desimone, 2009) entsprechen. Mit dem vorliegenden Manual intendieren wir einen Forschungsbeitrag zur Beschreibung von Fortbildungen als wichtigem Stimuli, um unterrichtliche Praxis zu verbessern (Richter, Kunter, Lüdtke, Klusmann, \& Baumert, 2011).

Neben der Erfassung von Veränderungen auf beobachtbarer Ebene, stellt die Beschreibung der Wahrnehmungen seitens der Lehrpersonen sowie der Schülerinnen und Schüler einen zentralen Bestandteil bei der Gesamtbetrachtung von unterrichtsbezogenen Veränderungen dar (Clarke \& Hollingsworth, 2002). Kapitel 5 stellt die im Projekt eingesetzten Fragebogeninstrumente vor, mithilfe derer schwerpunktmäßig die Wahrnehmung der Fortbildung durch die Lehrpersonen als Lerngelegenheit sowie Transfermöglichkeiten der dort erarbeiteten Inhalte erfasst wurden. Intention war es, die Lehrkräfte als individuelle Lerner zu 
betrachten und damit ihre persönlichen Einstellungen und Wahrnehmungen zu beschreiben. So wurden z.B. variierende Transferwahrnehmungen in die eigene unterrichtliche Praxis als wichtige Erklärungsgrundlage angenommen, um z.B. in der Fortbildungsforschung beschriebene ausbleibende Praxisveränderungen (van den Bergh, Ros, \& Beijaard, 2015; Vescio, Ross, \& Adams, 2008) besser zu verstehen.

In Kapitel 6 stellen wir zum Abschluss alle Skalen vor, die zur Befragung der Schülerinnen und Schüler zum Einsatz kamen. Im Einklang mit der bisherigen videobasierten Unterrichtsforschung (Seidel, Prenzel, Duit, \& Lehrke, 2003) war unser Anliegen - direkt nach der Unterrichtsvideographie - die Wahrnehmungen der Schülerinnen und Schüler $\mathrm{zu}$ dokumentieren, um einen triangulierenden Blick auf die Unterrichtsstunde legen zu können. In diesem Kontext war ein Ziel, möglichst proximal am Thema der Fortbildung zu erheben, weshalb einige aus der Literatur heraus entwickelte Skalen zur Wahrnehmung dialogischer Unterrichtsgesprächsführung aus Lernendenperspektive im Band vorgestellt werden. Die aus anderen Studien entnommenen Skalen zur Erfassung von stabilen Schülereigenschaften wurden in den Band mit aufgenommen, da sie sich vor allem zur Beschreibung und Interpretation differenzieller Wirkungen der Fortbildung auf Subgruppen von Schülerinnen und Schülern als bedeutsam erwiesen haben (Pehmer, Gröschner, \& Seidel, 2015b).

\section{Danksagung}

Wir bedanken uns bei allen teilnehmenden Lehrpersonen für ihre Offenheit und den Mut, in ihrer Interaktionspraxis etwas "Neues“ zu probieren sowie bei den Schülerinnen und Schülern für ihre Einschätzungen. Darüber hinaus bedanken wir uns bei allen Projektmitarbeiterinnen und -mitarbeitern beider Förderphasen, die zum Großteil auch als Autorinnen und Autoren an diesem Band beteiligt sind und maßgeblich zum Erfolg des Projekts beigetragen haben, sowie bei allen studentischen und wissenschaftlichen Hilfskräften. 


\section{Ricardo Böheim, Alexander Gröschner, Maralena Weil, Ann-Kathrin Schindler \& Tina Seidel}

\section{Konzeption der „Dialogue II"-Studie und Erhebungsablauf}

\subsection{Konzeption und Vorstellung der Fortbildungsangebote}

Im Rahmen des DFG-Projekts „Dialogue II“ wurden zwei Fortbildungsangebote zur Förderung der dialogischen Unterrichtsgesprächsführung entwickelt. Die inhaltliche Ausrichtung der beiden Fortbildungsangebote war identisch und wird im Folgenden näher erläutert. Für die terminologische Konzeptualisierung der dialogischen Gesprächsführung wurden die von Alexander (2018) formulierten fünf Prinzipien angewendet. Demnach sind dialogische Unterrichtsgespräche:

- kollektiv, d.h. Lehrpersonen und Lernende kooperieren und verfolgen ein gemeinsames Lernziel,

- reziprok, d.h. Lehrpersonen und Lernende übernehmen gemeinsam Verantwortung für den Lernprozess und Lernen von- und miteinander,

- unterstützend, d.h. Lernende werden in einer konstruktiven Gemeinschaft dazu eingeladen ihre Gedanken und Ideen frei zu äußern,

- kumulativ, d.h. Wissensaufbau ist ein Prozess der Ko-Konstruktion zwischen Lehrpersonen und Lernenden, bei dem ausgetauschte Ideen aufeinander aufbauen,

- zweckmäßig, d.h. das Unterrichtsgespräch ist auf festgelegte Lernziele ausgerichtet.

Ziel der Fortbildung war es ein Unterrichtsgespräch zu fördern, bei dem Lernende und Lehrpersonen sich als gleichwertige Konversationspartner verstehen, die durch den konstruktiven Austausch von Ideen und Gedanken effektives Lernen ermöglichen. In beiden Angeboten kam eine Toolbox zur Förderung der dialogischen Unterrichtsgesprächsführung zum Einsatz. Dabei wurden sowohl diskursfördernde Unterrichtsmethoden (talk activities) als auch Gesprächsstrategien (talk moves) vorgestellt, die sich in bisherigen Interventionsstudien als wirksam erwiesen haben. Diskursfördernde Unterrichtsmethoden (z.B. Meldekette oder Think-Pair-Share) sind niederschwellig umsetzbare Elemente, die Lehrpersonen dabei unterstützen sollen, Interaktionen im Unterrichtsgespräch schülerorientiert zu organisieren. Der ergänzende Einsatz von Gesprächsstrategien soll den Lehrpersonen dabei helfen, das Unterrichtsgespräch produktiv zu moderieren. Angelehnt an Michaels und O'Connor (2015) sollten mit Hilfe der Gesprächsstrategien vier zentrale Ziele gefördert werden:

1. Lernende ermutigen, ihre Ideen und Gedanken ausführlich zu erklären (z.B., „Kannst du das genauer erklären?")

2. Lernende ermutigen sich gegenseitig zuzuhören (z.B., „Wer kann nochmal zusammenfassen, was gerade gesagt wurde?")

3. Lernende ermutigen, ihre Ideen und Gedanken nachvollziehbar zu strukturieren (z.B., „Kannst du diese Aussage belegen?“)

4. Lernende ermutigen, von- und miteinander zu lernen (z.B., „Wer möchte Stellung zu dieser Antwort beziehen?)

Bei der Konzeption der Fortbildungsangebote wurden zentrale Merkmale effektiver Fortbildungen zu Grunde gelegt (Borko, Jacobs, \& Koellner, 2010; Desimone, 2009; 
Kennedy, 2016; Wilson, 2013). Auf Basis der Erkenntnisse dieser Forschungsliteratur (v.a. Desimone, 2009), wurde ein Fortbildungsangebot entwickelt,

a) bei dem die Lehrpersonen den eigenen Lernprozess aktiv mitgestalten können (active learning),

b) bei dem Gelegenheit zu Kooperation und kollegialem Austausch besteht (collective particitpation),

c) in dem unterrichtsrelevante Inhalte thematisiert werden (content focus),

d) das über einen längeren Zeitraum und über mehrere Sitzungen stattfindet (duration) und

e) in dem die thematische Ausrichtung der Fortbildung auf Bedürfnisse der Lehrpersonen abgestimmt ist (coherence).

Während die inhaltliche Ausrichtung beider Fortbildungsangebote gleich gehalten wurde, unterschieden sich die beiden Angebote in der Nutzung von Video als ein Medium zur Begleitung des Professionalisierungsprozesses der Lehrpersonen. Beide Fortbildungsprogramme hatten eine Gesamtdauer von 22 Stunden, die sich auf mehrere Workshops verteilten (siehe Abbildung 2.1). Beide Programme wurden jeweils von einem zusätzlichen Einführungsworkshop sowie einem Abschlussworkshop gerahmt.

Fortbildungsangebot 1 - Der Dialogische Videozirkel (DVC)

Das videobasierte Fortbildungskonzept wurde auf der Basis des empirisch erprobten Dialogischen Videozirkels, welcher in der ersten Förderphase des Projekts entwickelt wurde, angeboten (Gröschner et al., 2015). Bei diesem Ansatz werden (1) gemeinsame Unterrichtsplanung, (2) Unterrichtsvideographie und (3) Videoreflexion systematisch miteinander verknüpft. Die Lehrpersonen integrierten zunächst verschiedene Elemente der dialogischen Unterrichtsgesprächsführung in eine mitgebrachte Unterrichtsplanung, die gemeinsam mit Kolleginnen und Kollegen und der Fortbildungsleitung besprochen wurde. Im Anschluss unterrichteten die Lehrpersonen die geplante Unterrichtsstunde und diese wurde videographiert. Die Umsetzung wurde abschließend anhand von selektierten Videosequenzen in der Lerngemeinschaft reflektiert. Insgesamt wurden im Verlauf dieser videobasierten Fortbildung zwei dialogische Videozyklen von den teilnehmenden Lehrpersonen durchlaufen (siehe Abbildung 2.1).

Fortbildungsangebot 2 - Das dialogisch-instruktionale Programm (DIP)

Das dialogisch-instruktionale Fortbildungsprogramm umfasste zwei Tagesworkshops. Im ersten Workshop erhielten die Lehrpersonen eine Einführung in die dialogische Unterrichtsgesprächsführung, wobei Unterschiede und Parallelen zu den eigenen Diskursroutinen reflektiert wurden. Um den Bezug zur Unterrichtspraxis herzustellen, wurden anschließend verschiedene Elemente der dialogischen Gesprächsführung (z.B. Offenheit der Fragen, produktiver Umgang mit Schülerantworten) anhand von textbasierten Fallvignetten illustriert und deren Relevanz für den Unterricht gemeinsam diskutiert. Zur Wiederholung der vorgestellten Inhalte und zur Vorbereitung auf den zweiten Workshop erhielten die Lehrpersonen eine Reflexionsaufgabe. Im zweiten Tagesworkshop wurden authentische Lerngelegenheiten geschaffen, um Lehrpersonen bei der Umsetzung einer dialogischen Unterrichtsgesprächsführung zu unterstützen. In simulierten Gesprächssituationen konnten Lehrpersonen die vorgestellten Gesprächsstrategien (talk moves) und diskursfördernde Unterrichtsmethoden (talk activities) zum Einsatz bringen. Abschließend wurden die Erfahrungen aus diesen Rollenspielen gemeinsam reflektiert, um das Potential der dialogischen Gesprächsführung für den eigenen Unterricht zu verdeutlichen. 


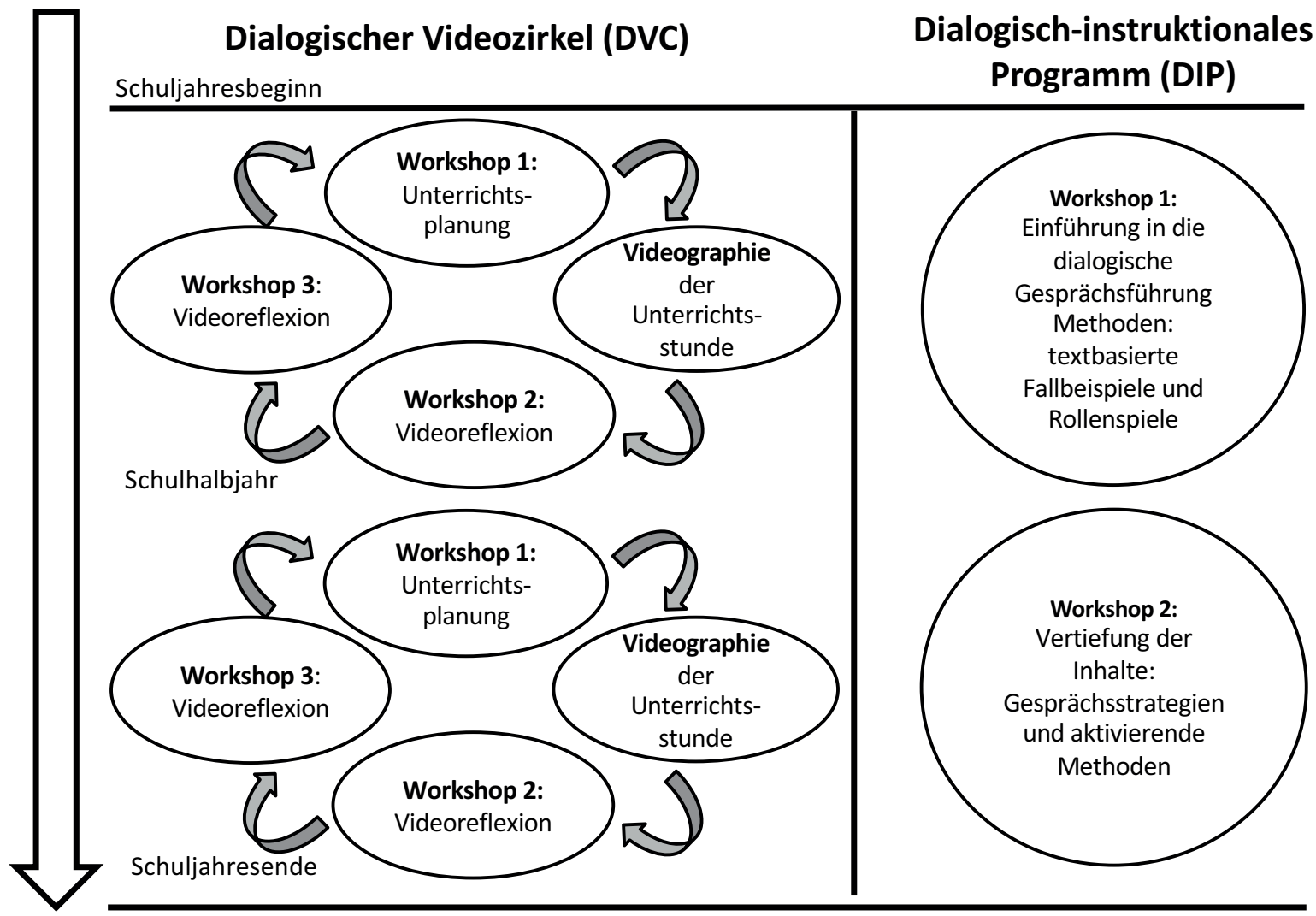

Abbildung 2.1: Übersicht der Fortbildungsworkshops

Gemäß der Designs der Variation der adaptiven vs. spezifischen Darstellung, sind Vermittlungslogik, Transferunterstützung und Inhalte im Dialogischen Videozirkel sehr stark auf die Bedarfe der Teilnehmenden ausgerichtet, während im dialogisch-instruktionalen Programm die gleichen Inhalte auf der Basis der bisherigen Forschung thematisiert und dargeboten wurden.

\subsection{Ablauf der Studie}

\subsubsection{Teilnehmende Lehrpersonen}

$\mathrm{Zu}$ Beginn der Studie wurden alle Gymnasien im Münchner Großraum über die Fortbildung zur Förderung der dialogischen Unterrichtsgesprächsführung informiert und zur Teilnahme eingeladen. Insgesamt nahmen $N=19$ Lehrpersonen an der Fortbildungsstudie teil (13 Lehrerinnen und 6 Lehrer). Das durchschnittliche Alter der Lehrpersonen betrug 38 Jahre $(S D=8.56)$. Ihre Lehrerfahrung lag zwischen zwei und 25 Jahren $(M=8.13, S D=6.53)$. Den teilnehmenden Lehrpersonen war es freigestellt zu entscheiden, mit welcher Klasse (Jahrgangstufe und Fach) der Sekundarstufe I sie an der Fortbildungsstudie teilnehmen wollten. Neben der Tatsache, dass diskursfördernde Unterrichtsmethoden und Gesprächsstrategien fächerübergreifende Bedeutung haben, wurde der interdisziplinäre Charakter der Fortbildung von den Lehrpersonen der ersten Förderphase (Dialogue I) beibehalten. Fünf Lehrpersonen wählten eine Klasse mit dem Fach Mathematik, neun Lehrpersonen wählten eine Klasse aus dem Fachbereich der Naturwissenschaften (Biologie, Chemie, Geographie oder Physik) und fünf Lehrpersonen wählten eine Klasse aus einem sprachlichen Fachbereich (Deutsch oder Englisch). Sieben der ge- 
wählten Klassen kamen aus der zehnten Jahrgangsstufe, weitere sieben der gewählten Klassen kamen aus der neunten Jahrgangsstufe und die übrigen fünf Klassen waren in der achten Jahrgangsstufe. Die dazugehörigen 450 Schülerinnen und Schüler ( $54 \%$ weiblich) waren durchschnittlich $M=14.59$ Jahre alt ( $S D=1.06)$.

Die teilnehmenden Lehrpersonen wurden nach der grundlegenden Anmeldung zur Fortbildung einem der beiden Angebote randomisiert zugewiesen. Insgesamt nahmen zehn Lehrpersonen am videobasierten Fortbildungsangebot teil, während neun Lehrpersonen am instruktionsbasierten Fortbildungsangebot teilnahmen. Für die Genehmigung der Studie wurden Einverständnisse von Lehrpersonen, Schülerinnen und Schülern, Eltern, der Schulleitung sowie dem Bayerischen Kultusministerium eingeholt.

\subsubsection{Erhebungsdesign}

Das Forschungsdesign ist in Abbildung 2.2 dargestellt. Im Verlauf des Schuljahres besuchten die Lehrpersonen die verschiedenen Workshops zum jeweiligen Fortbildungsangebot. In einem Prä-Post-Design wurden von allen neunzehn Lehrpersonen vor und nach der Teilnahme an der Interventionsstudie jeweils eine Unterrichtsstunde videographiert. Im Anschluss an die videographierte Unterrichtsstunde wurden sowohl Lernendenfragebögen (siehe Kapitel 6) als auch Lehrpersonenfragebögen (Kapitel 5) ausgehändigt. Zusätzlich wurde in der Mitte des Schuljahres eine weitere Befragung der Schülerinnen und Schüler sowie der Lehrpersonen durchgeführt. Die genauen Erhebungszeitpunkte der einzelnen Skalen sind in den jeweiligen Kapiteln 5 und 6 graphisch für das jeweilige Angebot und die Zielgruppe (Lernende bzw. Lehrpersonen) dargestellt.

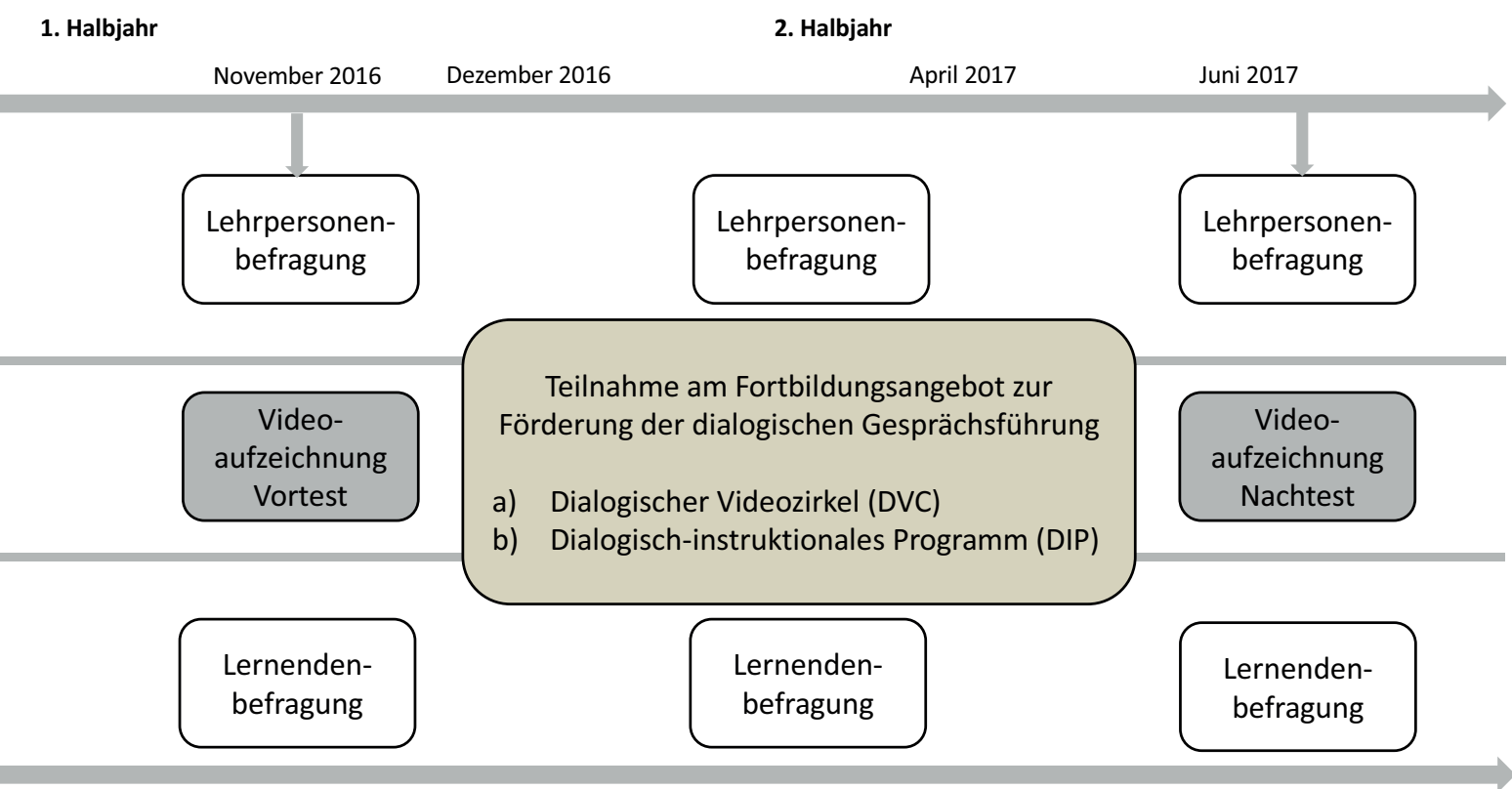

Abbildung 2.2: Übersicht Studienverlauf 


\subsection{Richtlinien zur Videoaufzeichnungen in „Dialogue II"}

Eine große Rolle bei der Erfassung von Videodaten spielt die Standardisierung. Beobachtungen sind stets durch die subjektive Wahrnehmung und Interpretation geprägt und bedürfen deshalb Kontrollen hinsichtlich ihrer Gültigkeit sowie Genauigkeit (Seidel et al., 2006). Um verschiedene Unterrichtsstunden miteinander vergleichen zu können, sind standardisierte Richtlinien bei der Videographie und Auswertung von Unterricht unverzichtbar (vgl. Häusler, Jurik, Schindler, Gröschner, \& Seidel, 2019).

In „Dialogue II“ orientierten wir uns an den folgenden Standards: Der Unterricht wurde sowohl aus einer auf die Klasse gerichteten Überblickskamera gefilmt als auch über eine zweite Kamera, welche die Interaktionen zwischen Lehrpersonen und den Schülerinnen und Schülern aufzeichnete (Lehrerkamera). Bei der Überblicksperspektive wurde die Kamera meist vorne neben der Tafel aufgestellt und frontal auf die Klasse gerichtet, um so das Unterrichtsgeschehen mit Blick auf die gesamte Klasse aufzuzeichnen. Die Lehrerkamera folgte im Unterrichtsverlauf hauptsächlich den Interaktionen der Lehrperson und wurde daher räumlich seitlich von der Übersichtskamera platziert. Diese verfolgte die Lehrperson, v.a. auch bei Interaktionen mit einzelnen Schülerinnen bzw. Schülern.

Auch die einzelnen Fortbildungsworkshops wurden videographiert, um die Interaktionen der Lehrpersonen untereinander sowie mit der Workshopleitung (Facilitator) zu beschreiben. Hierzu wurden zwei Kameras eingesetzt, die es ermöglichten, alle Lehrpersonen zu erfassen.

Die Abbildungen 2.3 und 2.4 zeigen den Kameraaufbau im Klassenzimmer bzw. bei den Fortbildungsworkshops im Rahmen beider Angebote.

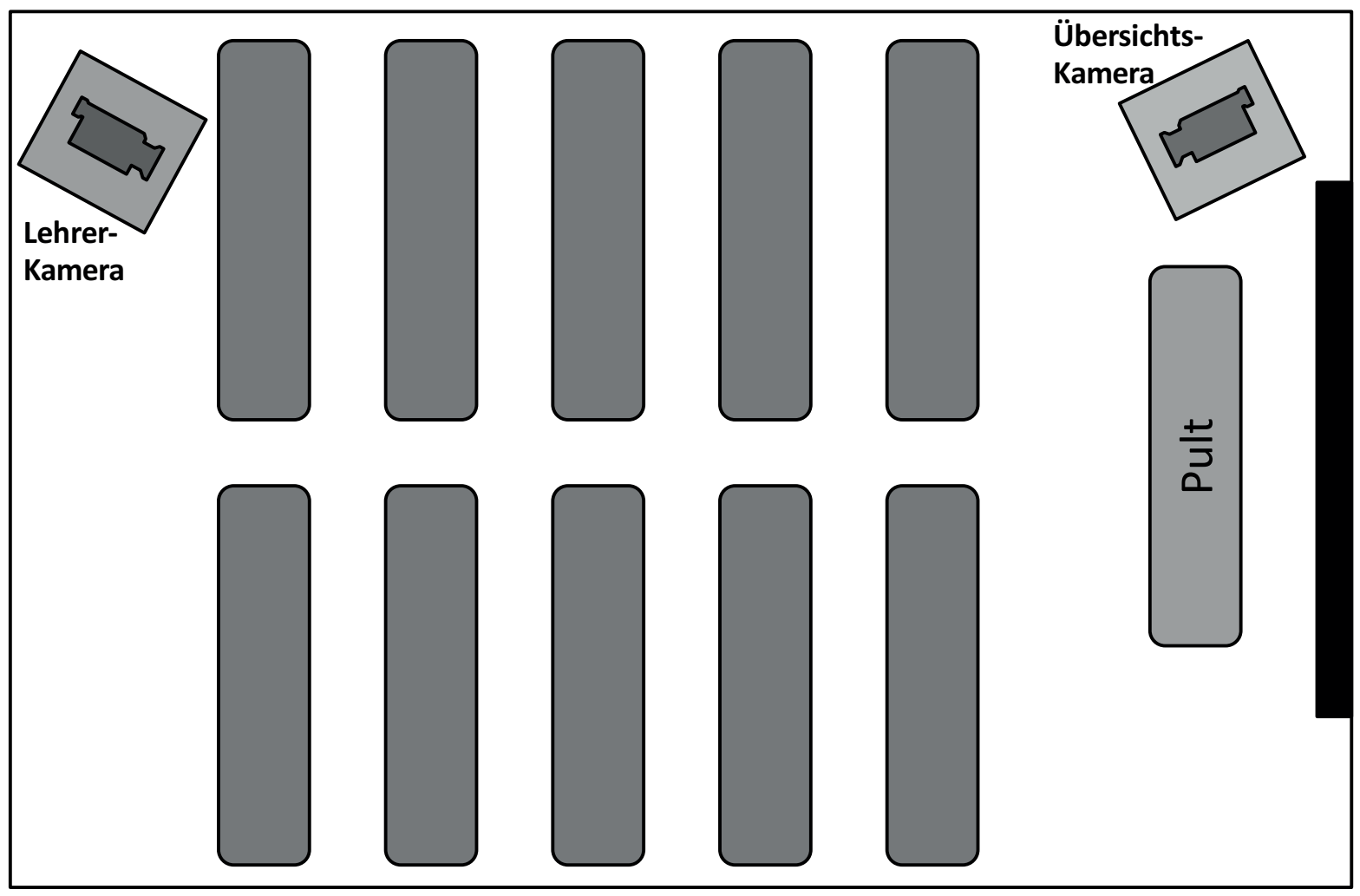

Abbildung 2.3: Kamerapositionen zur Videographie der Unterrichtsstunden in Klassenzimmern 


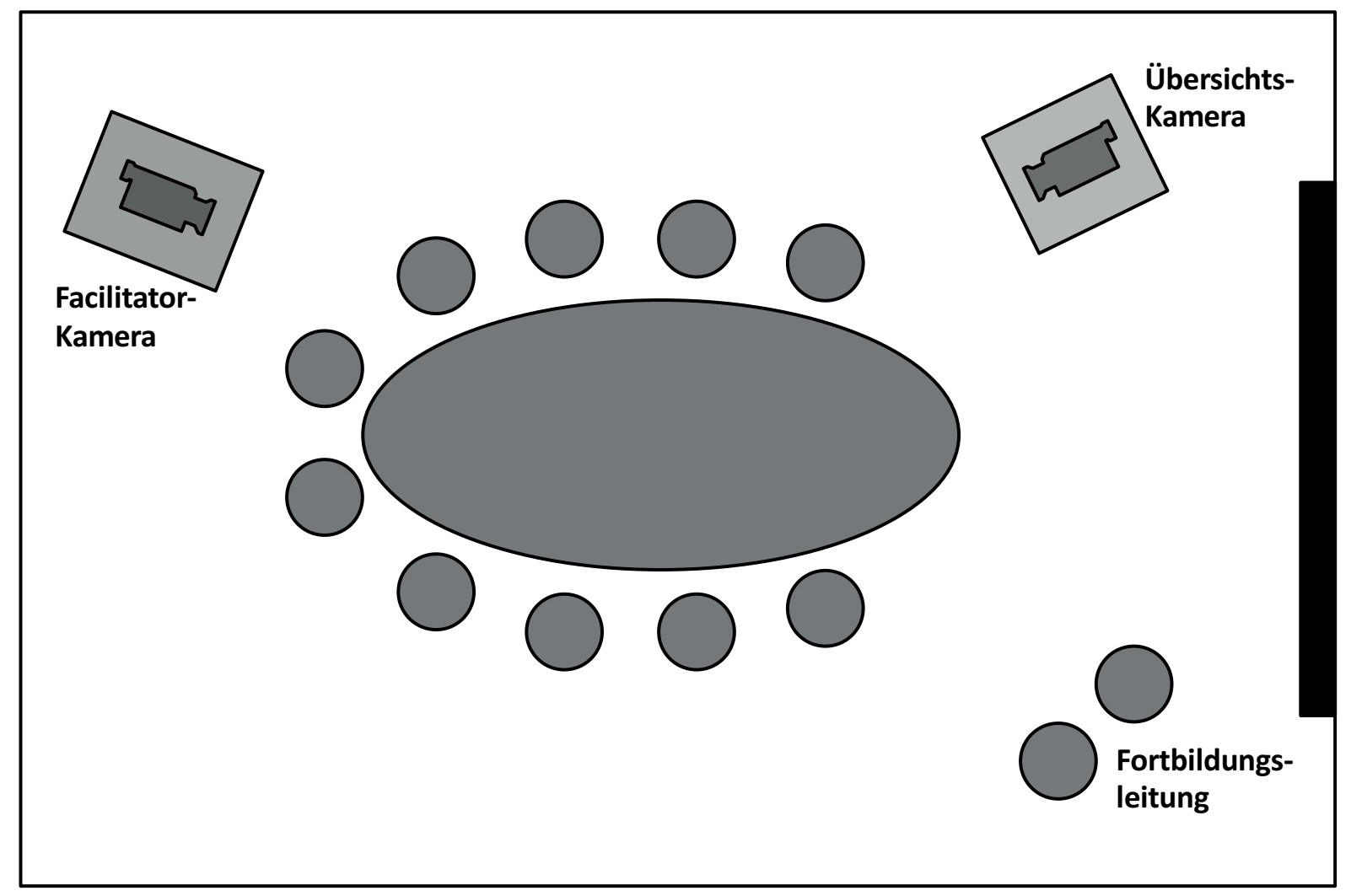

Abbildung 2.4: Kamerapositionen zur Videographie der Fortbildungsworkshops

\subsection{Technische Ausrüstung: Verwendete Hard- und Software in "Dialogue II"}

Im Folgenden wird die technische Ausrüstung (verwendete Kamera- und Mikrofontypen) des Projekts vorgestellt. Aufgrund des schnellen technologischen Fortschritts in diesem Bereich, sei darauf hingewiesen, dass die verwendete Ausrüstung nicht alle Qualitätsansprüche der aktuell verfügbaren Geräte in der Videound Audiographietechnik erfüllt (z.B. 4K-Auflösung). Dennoch dienen sie als grundlegende Orientierungshilfe, wie die technische Ausrüstung einer videobasierten Interventionsstudie aussehen kann.

\section{Verwendete Camcorder}

Die Aufnahme der Unterrichtsvideos wurde mit professionellen Camcordern der Marke JVC durchgeführt (Gerätetypus: GY-HM100E). Die verwendeten Camcorder verfügen über ein leistungsstarkes Objektiv mit einem 10fach optischen Zoom $(3,7 \mathrm{~mm}-37 \mathrm{~mm})$, sodass hochauflösende Nahaufnahmen vom geschehenen Unterricht (z.B. Tafelanschriebe) möglich waren. Um die gesamten Interaktionen im Klassenzimmer adäquat einzufangen, sind die Kameras zusätzlich mit einem Weitwinkelkonverter ausgestattet (Marke: JVC, Gerätetypus: GL-V0746, Brennweitenverlängerung: $0.7 \mathrm{x}$ ). Die Unterrichtsvideos wurden in Full-HD-Qualität (1080p, 25fps) im MP4-Dateiformat aufgezeichnet. Als Speichermedium kommen zwei SD-Karten zum Einsatz (2x32GB). Über die vorhandenen XLR Stecker lassen sich zwei externe Mikrofone anschließen und damit eine Audioaufnahme auf zwei getrennten Tonspuren realisieren. Zur Verbesserung der Bildqualität werden alle Videoaufnahmen mit Stativen durchgeführt. Die verwendeten Stative der Marke Manfrotto haben eine Maximallänge von 1,54 Metern (Typbezeichnung: 546B). 
Durch den hochwertigen Schwenkkopf mit Hydrodämpfung (Typenbezeichnung: 501 HDV) können sanfte Kamerabewegungen durchgeführt werden, ohne dass die Aufnahmen verwackeln. Die Stromversorgung der Camcorder wird über leistungsstarke Akkus sichergestellt (2300 mAh).

\section{Verwendete Mikrofone}

Für die Tonaufnahme kamen zwei Kondensatormikrofone zum Einsatz. Der verwendete Camcorder besitzt standardmäßig ein Kondensatormikrofon, das über eine Audioeinheit auf dem Camcorder befestigt wird und einen XLR-Anschluss besitzt. Es handelt sich dabei um ein Richtmikrofon, das darauf ausgelegt ist trotz Umgebungsgeräuschen ein gutes Tonsignal in Aufnahmerichtung zu erzeugen. Für die Tonaufnahme der Lehrperson kommt zusätzlich ein Lavaliermikrofon zum Einsatz. Dabei handelt es sich um ein Funkmikrofon der Marke Sennheiser (Gerätetypus: ew $100 \mathrm{G} 3$ ). Das kleine Ansteckmikrofon wird über ein Kabel mit einem Sender verbunden, der am Gürtel oder an der Hosentasche befestigt werden kann. Der aufgenommene Ton wird an den Empfänger gesendet, der ebenfalls an der Audioeinheit auf dem Camcorder befestigt ist und über einen XLRAnschluss mit dem Camcorder verbunden wird. Das kugelförmige Ansteckmikrofon ermöglicht eine hohe Aufnahmequalität der Stimme der Lehrperson (Nähe zur Sprecherquelle) und ist zudem hilfreich, um die Kommunikation zwischen Lehrpersonen und einzelnen Schülerinnen und Schülern aufzuzeichnen (z.B. bei individueller Lernbegleitung in Selbstlernphasen). Die Sender- und Empfängereinheit des Funkmikrofons wird mit Akkus betrieben.

\section{Videobearbeitungssoftware}

Zur Nachbearbeitung der Unterrichtsaufnahmen wurde die Videobearbeitungssoftware Adobe Premiere Pro CC verwendet. Neben einfachen Werkzeugen zum Videoschnitt können mit Hilfe dieses professionellen Nachbearbeitungsprogramms z.B. Überblickskamera und Lehrerkamera zusammengefügt werden, sodass beide Kameraperspektiven gleichzeitig betrachtet werden können. Zudem wurden die verschiedenen Tonspuren der beiden Kameras (Richt- und Funkmikrofon) synchronisiert und die Qualität des Tons bei Bedarf nachgebessert.

\section{Analyse der Unterrichtsvideos und deren Datenverarbeitung}

Die anschließende Analyse der Unterrichtsvideos wurde mit der von Mangold entwickelten Interact-Software (Mangold, 2017) durchgeführt. Die Unterrichtsaufnahmen wurden mit der Software in vier zehn-Minuten Sequenzen eingeteilt, die anschließend mit dem entwickelten Videomanual (siehe Kapitel 3) kodiert wurden. Die Ergebnisse der Videokodierung wurden zunächst als CSV-Dateien abgespeichert und anschließend in SPSS importiert, um sie mir den entsprechenden Fragebogendaten zusammenzuführen.

Die Auswertung der Fortbildungsvideos erfolgte ebenfalls mithilfe der Software Interact. Die Sequenzen wurden entsprechend der in Kapitel 4 beschriebenen Fortbildungsabschnitte (Moves) segmentiert und anschließend kodiert. Die Ergebnisse dieser Videokodierungen wurden ebenfalls als CSV-Dateien exportiert und anschließend mit SPSS ausgewertet. 
Ann-Kathrin Schindler, Ricardo Böheim, Maralena Weil, Alexander Gröschner \& Tina Seidel

\section{Videoinstrument „Dialogische Unterrichtsgesprächsführung" - Auswertung der Unterrichtsvideos}

\subsection{Prämissen zur Entwicklung des Instruments}

Für die Entwicklung des Videoinstruments zur Auswertung der dialogischen Unterrichtsgesprächsführung wurden zunächst Prämissen herausgearbeitet. Diese sollten eine Zielgerichtetheit, aktuelle Verortung in der bisherigen Forschung sowie eine geteilte Sichtweise des auswertenden Forschungsteams gewährleisten.

In Tabelle 3.1 werden sechs Prämissen vorgestellt, die maßgeblich zur Struktur und Konzeption des Videoinstruments beigetragen haben. Die einzelnen Prämissen werden den Zielen des Dialogue-II-Projekts zugeordnet; verdeutlicht zu welcher Forschungsrichtung diese Ziele gehören; und abschließend dargestellt, wie diese Überlegungen die Gestaltung des Videoinstruments beeinflusst haben. Diese Überlegungen haben dazu geführt, dass die Unterrichtsvideos für die Analyse in 10 Minuten-Segmente eingeteilt wurden (ökonomisch); die Items nicht nur das Handeln der Lehrpersonen erfassen, sondern ebenso das Handeln der Schülerinnen und Schüler berücksichtigt wurde (verknüpfend); die Abstufung der Ausprägungen sowohl eine Tendenzeinschätzung als auch eine Feineinschätzung erforderten (messgegenstandsgerecht); die Formulierung der Ausprägungsstufen die situationsbedingte Adaptivität der Lehrpersonen berücksichtigt (bedarfsgerecht); die induktiv hergeleiteten Items durch deduktiv abgeleitete Elemente erweitert wurden (z.B. welche Methoden zum Einsatz kamen); verschiedene Forschungstraditionen (Unterrichtsqualitätsforschung und dialogische Unterrichtskommunikation) zusammengeführt wurden. 
Tabelle 3.1: Prämissen zur Entwicklung des Kodiermanuals zur Auswertung der Unterrichtsvideos

\begin{tabular}{|c|c|c|c|c|}
\hline Prämisse & $\begin{array}{c}\text { Dialogue zielt auf } \\
\text { die Veränderung } \\
\text { von ... ab. }\end{array}$ & $\begin{array}{c}\text { Geteilte Sichtweise } \\
\text { des Forschungs- } \\
\text { teams }\end{array}$ & Forschungsstrang & $\begin{array}{l}\text { Ableitung für das } \\
\text { Videoinstrument }\end{array}$ \\
\hline Ökonomisch & $\begin{array}{l}\text {... Elementen des } \\
\text { produktiven Unter- } \\
\text { richtsgesprächs im } \\
\text { Rahmen der gesam- } \\
\text { ten Unterrichtsstun- } \\
\text { de. }\end{array}$ & $\begin{array}{l}\text { Fokus auf der Er- } \\
\text { fassung forschungs- } \\
\text { fragenrelevanter } \\
\text { Elemente. }\end{array}$ & $\begin{array}{l}\text { Ökonomisierung von } \\
\text { Videoforschung } \\
\text { (Begrich, Fauth, Kun- } \\
\text { ter, \& Klieme, 2017) }\end{array}$ & $\begin{array}{l}\text { Segmentierung } \\
\text { der gesamten } \\
\text { Unterrichtsstunde } \\
\text { in 10-minutige } \\
\text { Segmente. }\end{array}$ \\
\hline Verknüpfend & $\begin{array}{l}\text {... Lehrer- und Schü- } \\
\text { lerhandeln als sich } \\
\text { zwei bedingende } \\
\text { Handlungsweisen }\end{array}$ & $\begin{array}{l}\text { Lehrerhandeln und } \\
\text { Schülerhandeln sind } \\
\text { nicht technisch ab- } \\
\text { gegrenzt, sondern } \\
\text { bedingen sich. }\end{array}$ & $\begin{array}{l}\text { Dialogische Unter- } \\
\text { richtsgesprächsfüh- } \\
\text { rung (Reznitskaya, } \\
\text { Wilkinson, Oyler, } \\
\text { Bourdage-Reninger, } \\
\text { \& Sykes, 2016) }\end{array}$ & $\begin{array}{l}\text { Items werden so- } \\
\text { wohl für das Han- } \\
\text { deln der Lehrperson } \\
\text { als auch der Schüle- } \\
\text { rinnen und Schüler } \\
\text { ausformuliert. }\end{array}$ \\
\hline $\begin{array}{l}\text { Messgegen- } \\
\text { standsgerecht }\end{array}$ & $\begin{array}{l}\text {.. produktiven } \\
\text { Gesprächsführun- } \\
\text { gen mit einfachen } \\
\text { Techniken wie Ge- } \\
\text { sprächsstrategien }\end{array}$ & $\begin{array}{l}\text { Berücksichtigung } \\
\text { kleiner Veränderun- } \\
\text { gen im Handeln von } \\
\text { professionellenLehr- } \\
\text { personen. }\end{array}$ & $\begin{array}{l}\text { Einsatzbereiche von } \\
\text { Videoinstrumenten } \\
\text { (Reznitskaya, Wil- } \\
\text { kinson, Oyler et al., } \\
\text { 2016) }\end{array}$ & $\begin{array}{l}\text { Ausprägung der } \\
\text { Ratingskala } 6 \text { stufig } \\
\text { um zunächst eine } \\
\text { Tendenzeinschät- } \\
\text { zung (niedrig, mittel, } \\
\text { hoch), gefolgt von } \\
\text { einer Feineinschät- } \\
\text { zung vornehmen zu } \\
\text { können. }\end{array}$ \\
\hline Bedarfsgerecht & $\begin{array}{l}\text {... explizitem Wissen } \\
\text { über das produktive } \\
\text { Unterrichtsgespräch } \\
\text { ab, das bedarfsge- } \\
\text { recht zum Einsatz } \\
\text { gebracht wird }\end{array}$ & $\begin{array}{l}\text { Lehrpersonen ent- } \\
\text { scheiden in ihrem } \\
\text { Handeln explizit und } \\
\text { verstehen sich als } \\
\text { Facilitator des Unter- } \\
\text { richtsgesprächs. } \\
\text { Veränderungen } \\
\text { werden nicht um der } \\
\text { Veränderungwillen } \\
\text { vorgenommen. }\end{array}$ & $\begin{array}{l}\text { Dialogisches Lehren } \\
\text { und Lernen (Hattie } \\
\text { \& Timperley, 2007; } \\
\text { Reznitskaya, Wilkin- } \\
\text { son, \& Oyler, 2016; } \\
\text { Walshaw \& Anthony, } \\
\text { 2008) }\end{array}$ & $\begin{array}{l}\text { Ausprägung der } \\
\text { Ratingskala } \\
\text { nicht vorhanden (0) } \\
\text { bis adaptiv (5) }\end{array}$ \\
\hline $\begin{array}{l}\text { Induktiv und } \\
\text { deduktiv }\end{array}$ & $\begin{array}{l}\text {... explizitem theore- } \\
\text { tischem Wissen zum } \\
\text { produktiven Unter- } \\
\text { richtsgespräch und } \\
\text { die praktische Um- } \\
\text { setzung }\end{array}$ & $\begin{array}{l}\text { Lehrpersonen lernen } \\
\text { aus der Theorie UND } \\
\text { der Praxis der ande- } \\
\text { ren. }\end{array}$ & $\begin{array}{l}\text { Videobasiertes } \\
\text { Lernen von Lehrper- } \\
\text { sonen (u.a. Borko, } \\
\text { Jacobs, Eiteljorg, \& } \\
\text { Pittman, 2008) }\end{array}$ & $\begin{array}{l}\text { Entwicklung induk- } \\
\text { tiver und deduktiver } \\
\text { Ratingelemente. }\end{array}$ \\
\hline $\begin{array}{l}\text { Forschungs- } \\
\text { stränge } \\
\text { verknüpfend }\end{array}$ & $\begin{array}{l}\text {... dialogische Unter- } \\
\text { richtsgesprächsfüh- } \\
\text { rung als Unterrichts- } \\
\text { qualitätsmerkmal }\end{array}$ & $\begin{array}{l}\text { Lehrpersonen lernen } \\
\text { über Angebotsele- } \\
\text { mente der Unter- } \\
\text { richtsgesprächs- } \\
\text { führung in Form von } \\
\text { Sicht- und Tiefen- } \\
\text { strukturen. }\end{array}$ & $\begin{array}{l}\text { Unterrichtsqualitäts- } \\
\text { forschung (Kunter } \\
\text { \& Trautwein, 2013; } \\
\text { Seidel \& Reiss, 2014) } \\
\text { Dialogische Unter- } \\
\text { richtsgesprächs- } \\
\text { führung (Alexander, } \\
\text { 2005; Michaels } \\
\text { \& O'Connor, 2012; } \\
\text { Resnick et al., 2015) }\end{array}$ & $\begin{array}{l}\text { Forschungsverknüp- } \\
\text { fendes Ratingtool, } \\
\text { das den aktuellen } \\
\text { Forschungsstand } \\
\text { der Unterrichtsquali- } \\
\text { tätsforschung und } \\
\text { Forschung zur dialo- } \\
\text { gischen Unterrichts- } \\
\text { gesprächsführung } \\
\text { fusioniert. }\end{array}$ \\
\hline
\end{tabular}




\subsection{Theoretische Herleitung}

\section{Strukturierung des Instruments}

Wie sind Ereignisse im Unterrichtsgespräch genestet?

Zur Strukturierung des Instruments wird der Ansatz von Gee und Green (1998) (zitiert nach Hennessy, Drummond, Higham, Márquez, Maine, Ríos, García-Carrión, Torreblanca, \& Barrera, 2016) gewählt. Dabei werden Ereignisse in Gespräche auf drei Ebenen betrachtet: Das Makrolevel (kommunikative Situation) beschreibt den Kontext, in welchem ein Gespräch stattfindet (im Falle von Dialogue-IIKontext Unterricht); das Mesolevel (kommunikatives Event) hinterfragt z.B. Teilnehmerstruktur, Zweck oder Aufgabe des Gesprächs; das Microlevel (kommunikativer Akt) betrachtet die interaktionale Funktion des Gesprächs.

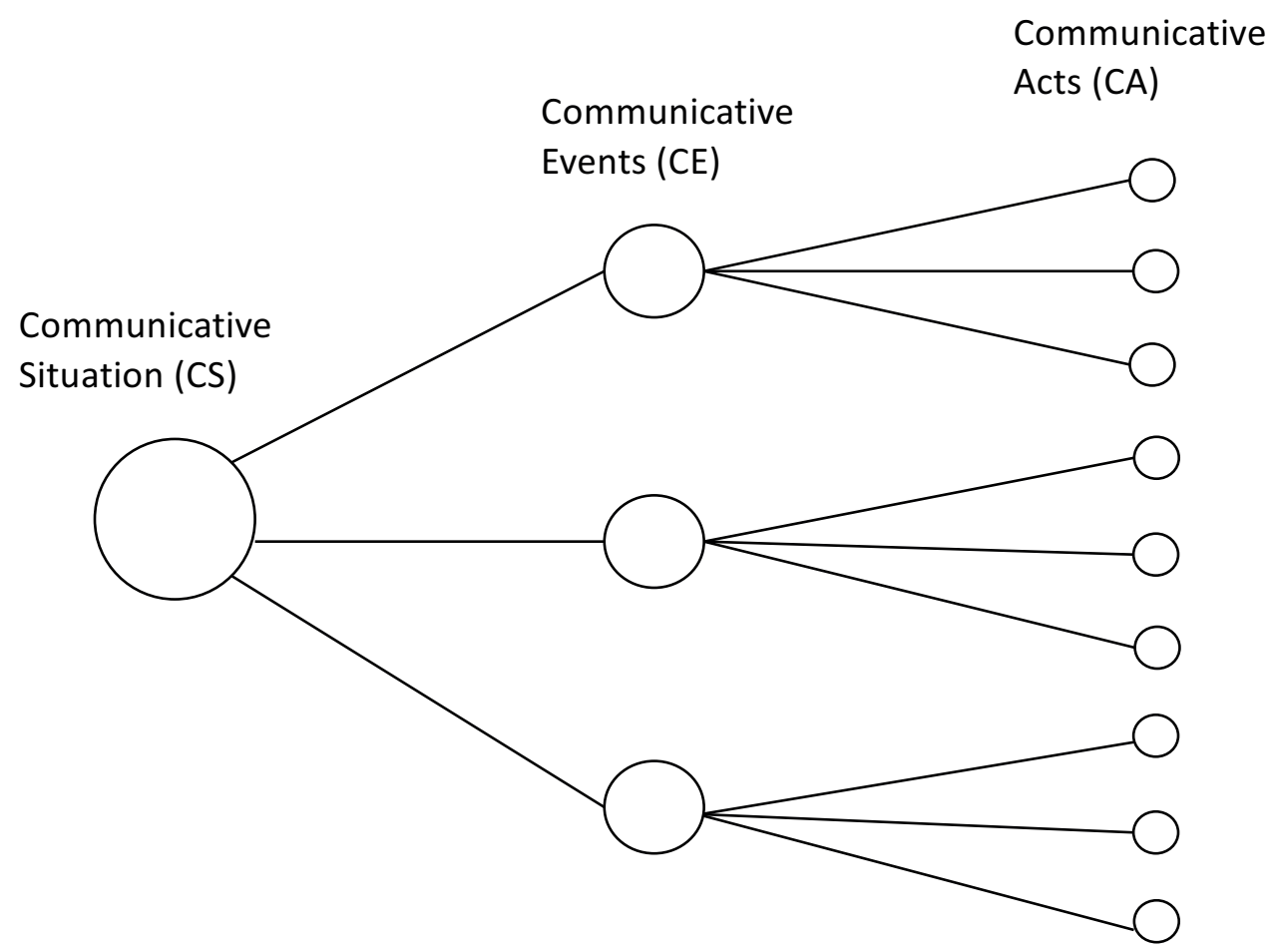

Abbildung 3.1: Nestung von Ereignissen im Unterrichtsgespräch (Hennessy et al., 2016)

Makrolevel (Kommunikative Situation)

Wie kann der Kontext des Unterrichtsgesprächs systematisch beschrieben werden?

Zur systematischen Beschreibung des Kontexts des Unterrichtsgesprächs wurde für jede Unterrichtsvideographie eine Kontextualisierung der kommunikativen Situation "Unterricht" vorgenommen. Dabei wird das Fach, das Thema der Stunde, das erkennbare Lernziel, sowie die mediale Unterstützung des Unterrichtsgesprächs festgehalten.

Mesolevel (Kommunikative Events)

Auf welche Art und Weise sind Akteure am Unterrichtsgespräch beteiligt?

Zur Charakterisierung der Beteiligung von Lehrperson und Schülerinnen und Schülern an kommunikativen Events wird das Verständnis der Unterrichtsqualitätsforschung herangezogen, dass Unterricht als Angebots-Nutzungsstruktur versteht (Seidel, 2014). Es wird zwischen lehrerzentrierter Instruktion, lehrerzentrierter 


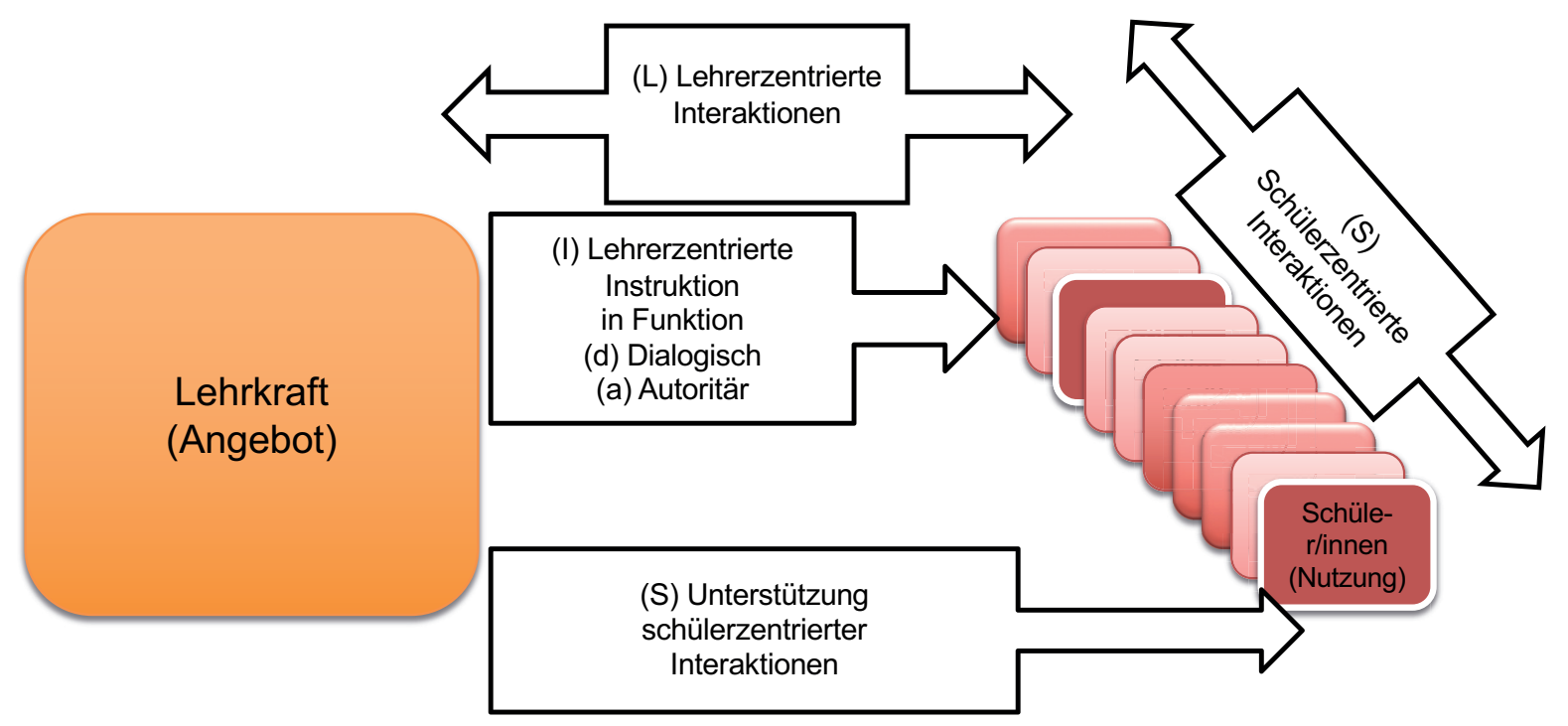

Abbildung 3.2: Angebots-Nutzungsstruktur im Unterrichtsgespräch (nach Seidel \& Reiss, 2014)

Interaktion und schülerzentrierter Interaktion (mit ggf. Lehrkraftunterstützung) unterschieden (detaillierte Ausführung siehe Abschnitt 3.3). Die Begrifflichkeit „Interaktion“ wird anhand der Überblicksarbeit von Chi (2009) gewählt.

Um Beteiligung der Schülerinnen und Schüler zu ermöglichen, stehen Lehrpersonen Tools sowohl aus der Sicht- als auch der Tiefenstrukturebene von Unterricht zu Verfügung. In diesem Verständnis wurden die Inhalte der Fortbildung "Dialogue II" in der Unterteilung "diskursfördernde Unterrichtsmethoden“ (Sichtstrukturebene) und "Gesprächsstrategien“ (Tiefenstrukturebene) aufbereitet. Zur Überprüfung des Transfers in die Unterrichtspraxis wird diese Betrachtungsweise der Unterrichtsqualitätsforschung beibehalten.

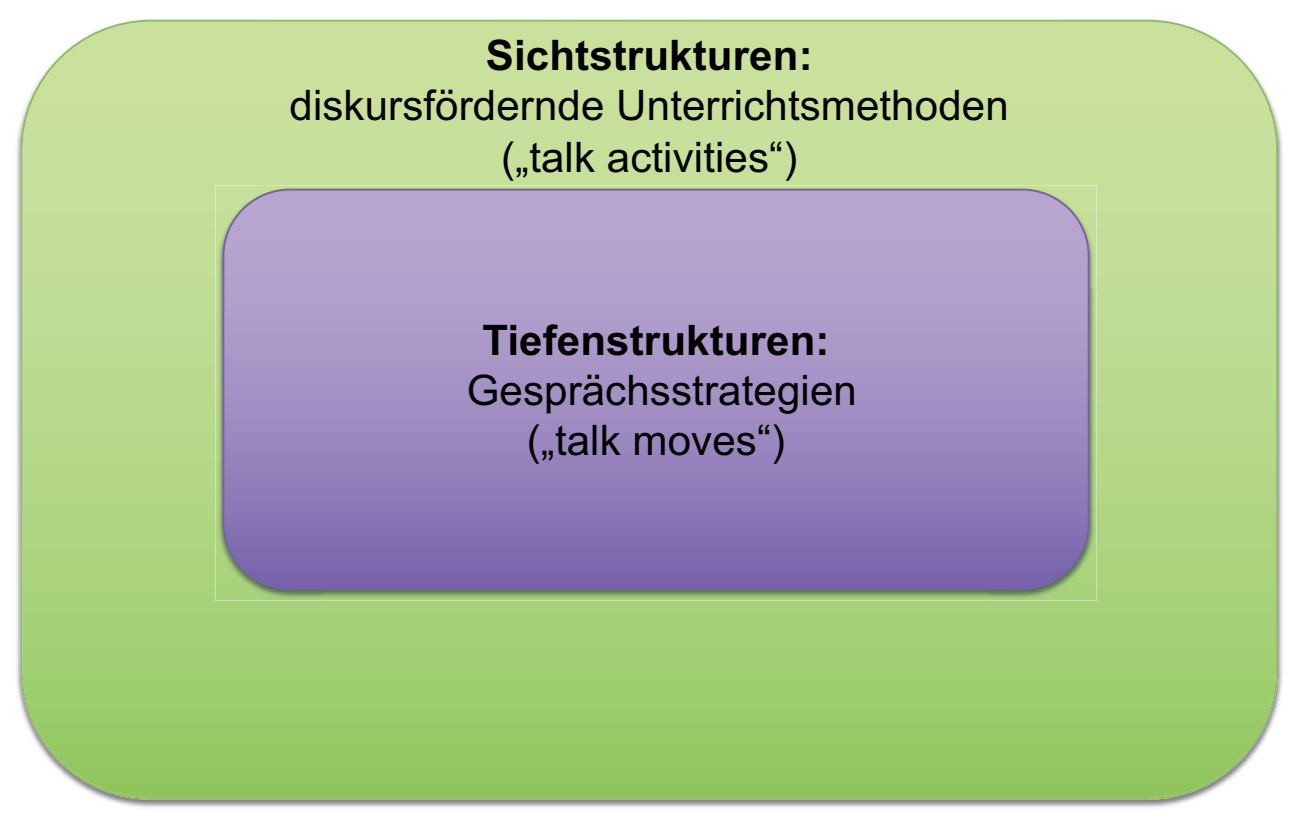

Abbildung 3.3: Sicht- und Tiefenstrukturen des Unterrichtsgesprächs (u.a. Gomez Zaccarelli, Schindler, Borko, \& Osborne, 2018; Kunter \& Trautwein, 2013; Michaels \& O'Connor, 2012) 
Mikrolevel (Kommunikativer Akt)

Welche Qualitätskriterien müssen im dialogischen Unterrichtsgespräch Berücksichtigung finden?

Entlang der Struktur des Ratinginstruments ART (Reznitskaya et al., 2016) wurden für das Dialogue-II-Ratingtool vier zentrale Qualitätsdimensionen des produktiven Unterrichtsgesprächs herausgearbeitet (siehe Tabelle 3.2).

Tabelle 3.2: Qualitätsdimensionen des produktiven Unterrichtsgesprächs (Alexander, 2005; Resnick, Michaels, \& O'Connor, 2010; Reznitskaya, Wilkinson, \& Oyler, 2016; Walshaw \& Anthony, 2008)

\begin{tabular}{|c|c|c|}
\hline $\begin{array}{l}\text { Qualitäts- } \\
\text { dimension }\end{array}$ & Beschreibung & Operationalisierung \\
\hline $\begin{array}{l}\text { \#0 Methodische } \\
\text { Unterstützung des } \\
\text { Unterrichtsge- } \\
\text { sprächs }\end{array}$ & $\begin{array}{l}\text { "Das produktive Unter- } \\
\text { richtsgespräch wird } \\
\text { durch eine Methode } \\
\text { unterstützt/angeregt/ } \\
\text { erhalten." }\end{array}$ & $\begin{array}{l}\text { Jede vorkommende Methode wird benannt und hinsichtlich } \\
\text { ihres adaptiven Transfers (Kein Nutzen/prozedurale Anwen- } \\
\text { dung/adaptive Anwendung) eingestuft. }\end{array}$ \\
\hline $\begin{array}{l}\text { \#1 zielgerichtet } \\
\text { und strukturiert }\end{array}$ & $\begin{array}{l}\text { „Das Unterrichtsge- } \\
\text { spräch verfolgt nachvoll- } \\
\text { ziehbar und strukturiert } \\
\text { ein Lernziel.“ }\end{array}$ & $\begin{array}{l}\text { 1.1 Für das Unterrichtsgespräch gibt es ein Lehr-Lernziel } \\
\text { (GOAL). } \\
\text { 1.2 Das Unterrichtsgespräch ist nachvollziehbar } \\
\text { (STRUCTURE). } \\
\text { 1.3 Die Anforderungen für das Erreichen des Lernziels im } \\
\text { Unterrichtsgespräch sind klar (CLARITY). }\end{array}$ \\
\hline $\begin{array}{l}\text { \# } 2 \text { aktivierend } \\
\text { und offen }\end{array}$ & $\begin{array}{l}\text { „Das Unterrichtsge- } \\
\text { spräch läd zur aktiven } \\
\text { Teilnahme ein und ist } \\
\text { offen für die Teilnahme } \\
\text { verschiedener Interak- } \\
\text { tionspartner mit unter- } \\
\text { schiedlichen Beiträgen.“ }\end{array}$ & $\begin{array}{l}\text { 2.1 Das Unterrichtsgespräch bietet Möglichkeiten für die akti- } \\
\text { ve Beteiligung von Schüler/innen (VERBAL ACTIVE). } \\
\text { 2.2 Das Unterrichtsgespräch bietet Möglichkeiten für ak- } \\
\text { tives gegenseitiges Zuhören von Schüler/innen (VERBAL } \\
\text { PASSIVE). } \\
\text { 2.3 Im Unterrichtsgespräch wird durch offene Fragen und } \\
\text { Aufgaben für verschiedene Antworten/Lösungen/Mei- } \\
\text { nungen/Elaborationen/Stellungsnahmen/Fragen aktiviert } \\
\text { (INITIATION). }\end{array}$ \\
\hline $\begin{array}{l}\text { \#3 interaktiv und } \\
\text { kumulativ }\end{array}$ & $\begin{array}{l}\text { „Im Unterrichtsgespräch } \\
\text { sind Lehrkraft und Schü- } \\
\text { ler gleichwertige Interak- } \\
\text { tionspartner und bauen } \\
\text { ihre Beiträge aufeinander } \\
\text { auf." }\end{array}$ & $\begin{array}{l}\text { 3.1 Für das Unterrichtsgespräch stellen elaborierte Schüler- } \\
\text { antworten/Begründungen/Erklärungen eine wichtige Res- } \\
\text { source dar (FOLLOW). } \\
\text { 3.2 Im Unterrichtsgespräch bauen Schülerbeiträge aufeinan- } \\
\text { der auf (LINK). }\end{array}$ \\
\hline $\begin{array}{l}\text { \#4 unterstützend } \\
\text { und nachhaltig }\end{array}$ & $\begin{array}{l}\text { „Im Unterrichtsgespräch } \\
\text { werden Unterstützungen } \\
\text { angeboten, um Schüler/ } \\
\text { innen in ihrem Lernen } \\
\text { weiter zu bringen." }\end{array}$ & $\begin{array}{l}\text { 4.1 Im Unterrichtsgespräch gibt es Hilfestellungen/Hinweise/ } \\
\text { Unterstützung/Feedback für das weitere Lernen/das Erreichen } \\
\text { des Lernziels (SCAFFOLD). } \\
4.2 \text { Im Unterrichtsgespräch dürfen Fehler gemacht werden } \\
\text { und werden als Ressource verstanden (MISTAKE). }\end{array}$ \\
\hline
\end{tabular}




\subsection{Kodierungsverfahren und Skalen}

\section{Schritt 1: Segmentierung zur Schaffung kommunikativer Events}

Die gesamte Unterrichtsstunde wurde für die Datenanalyse herangezogen, da die Fortbildung auf Methoden und Gesprächsstrategien zur Aktivierung und Unterstützung von Schülerinnen und Schülern abzielt, welche an unterschiedlichen Stellen in den Unterrichtsverlauf integriert werden können. Des Weiteren stellte die „Unterrichtsplanung“ der gesamten zu videographierenden Unterrichtsstunde einen wesentlichen Bestandteil des Fortbildungskonzepts dar.

Unter der Prämisse der Ökonomie wurden die Unterrichtsvideos in 10 Minuten Segmente mithilfe der Software Interact (Mangold, 2017) unterteilt und die ersten $4 \times 10$ Minuten mithilfe des Ratinginstruments eingestuft. Die Unterteilung begann ab dem offiziellen Start des Unterrichts (z.B. „Guten Morgen“). Die Zeiteinheit von 10 Minuten wird aus folgenden Gründen gewählt:

In internationalen Auswertungen zum Thema Unterrichtsqualität/produktives Unterrichtsgespräch werden Einheiten unterschiedlicher Länge gewählt: 10 Minuten (Seidel et al., 2003); 15 Minuten (Osborne et al., 2016); 20 Minuten (Reznitskaya, Wilkinson, \& Oyler, 2016), abgestimmt jeweils auf das Anliegen der Fortbildung (z.B. Reznitskaya, Wilkinson, \& Oyler, 2016: eine zentrale längere Diskussion in der Mitte der Unterrichtsstunde) und, um den Ratern eine ausreichende Beobachtungsgrundlage für die zugrunde liegenden Analyseschwerpunkte (z.B. Forschungsfragen) zu Verfügung zu stellen. In der Studie „Dialogue II“ wurden 10 Minuten als eine angemessene Einheit gewählt, welche ökonomisch handhabbar ist, sowie ausreichend Interaktionen zeigt, um zu einer Aussage über deren Qualität zu kommen.

\begin{tabular}{|l|l|l|l|}
\hline $10 \min$ & $10 \min$ & $10 \min$ & $10 \min$ \\
\hline & & & \\
& & & \\
\hline
\end{tabular}

\section{Schritt 2: Einordnung der kommunikativen Events in ihre Angebots- Nutzungsstruktur}

Alle 10-Minuten-Segmente werden als kommunikative Events betrachtet und in ihre Angebots-Nutzungsstruktur kategorisiert. Für die Zuordnung zur entsprechenden Angebots-Nutzungsstruktur ist entscheidend, wer für die Schülerinnen und Schüler der Hauptgesprächspartner ist (Lehrperson oder Mitschüler/innen). Dabei kann jedes Segment beiden Interaktionsformen (lehrerzentrierte und schülerzentrierte Interaktionen) zugordnet werden. Folgende Einstufungen standen zur Verfügung: 


\begin{tabular}{|l|l|}
\hline $\begin{array}{l}\text { Nur schülerzentrierte } \\
\text { Interaktionen (S) }\end{array}$ & $\begin{array}{l}\text { Diese Ausprägung wird auch gewählt, wenn zu Beginn des Segments organisatori- } \\
\text { sche Momente zu beobachten sind (z.B. Schüler/innen sammeln sich an Gruppen- } \\
\text { tischen) und die Lehrkraft währenddessen instruiert. Die instruierenden Lehrermo- } \\
\text { mente werden einer Wertung unterzogen, wenn sie nach entsprechender Definition } \\
\text { als Instruktion eingeordnet werden können. Das Rating zur Rolle der Lehrerinstruk- } \\
\text { tion wird auf der folgenden Seite 25 beschrieben. }\end{array}$ \\
\hline $\begin{array}{l}\text { Mehr schülerzentrierte } \\
\text { Interaktionen (S+) }\end{array}$ & $\begin{array}{l}\text { Diese Ausprägung ist zu wählen, wenn mehr Zeitanteil auf schülerzentrierte Interak- } \\
\text { tionen (z.B. 8 Minuten) ausfällt als auf lehrerzentrierte Interaktionen (z.B. } 2 \text { Minuten). }\end{array}$ \\
\hline Ausgewogen (0) & $\begin{array}{l}\text { Diese Ausprägung ist zu wählen, wenn beide Angebots-Nutzungsstrukturen in etwa } \\
\text { ausgewogen vorhanden sind. }\end{array}$ \\
\hline $\begin{array}{l}\text { Mehr lehrerzentrierte } \\
\text { Interaktionen (L+) }\end{array}$ & $\begin{array}{l}\text { Diese Ausprägung ist zu wählen, wenn mehr Zeitanteil auf lehrerzentrierte Inter- } \\
\text { aktionen (z.B. 8 Minuten) ausfällt als auf schülerzentrierte Interaktionen (z.B. 2 Minu- } \\
\text { ten). }\end{array}$ \\
\hline $\begin{array}{l}\text { nur lehrerzentrierte } \\
\text { Interaktionen (L) }\end{array}$ & $\begin{array}{l}\text { Diese Ausprägung wird auch gewählt, wenn zu Beginn des Segments organisato- } \\
\text { rische Momente zu beobachten sind, so dass anschließend lehrerzentrierte Inter- } \\
\text { aktionen stattfinden können (z.B. die Schüler/innen bewegen sich nach einer Grup- } \\
\text { penarbeitsphase zurück an ihre Plätze). }\end{array}$ \\
\hline
\end{tabular}

Im Folgenden sind die konkreten Beobachtungskriterien für schülerzentrierte und lehrerzentrierte Interaktionen beschrieben. Zudem werden konkrete Kriterien zur Einstufung instruktionaler Momente seitens der Lehrperson dargestellt. Unter Instruktion (I) wurden Momente verstanden, in welchen die Lehrperson die Interaktion unterbricht, um eine längere auf Lernaktivitäten bezogene Präsentation/Zusammenfassung/Ausführung/Erklärung/Tafelanschrieb vorzunehmen. Diese instruktionalen Momente wurden hinsichtlich ihrer Funktion (autoritär bis dialogisch) für das Unterrichtsgespräch bewertet. 


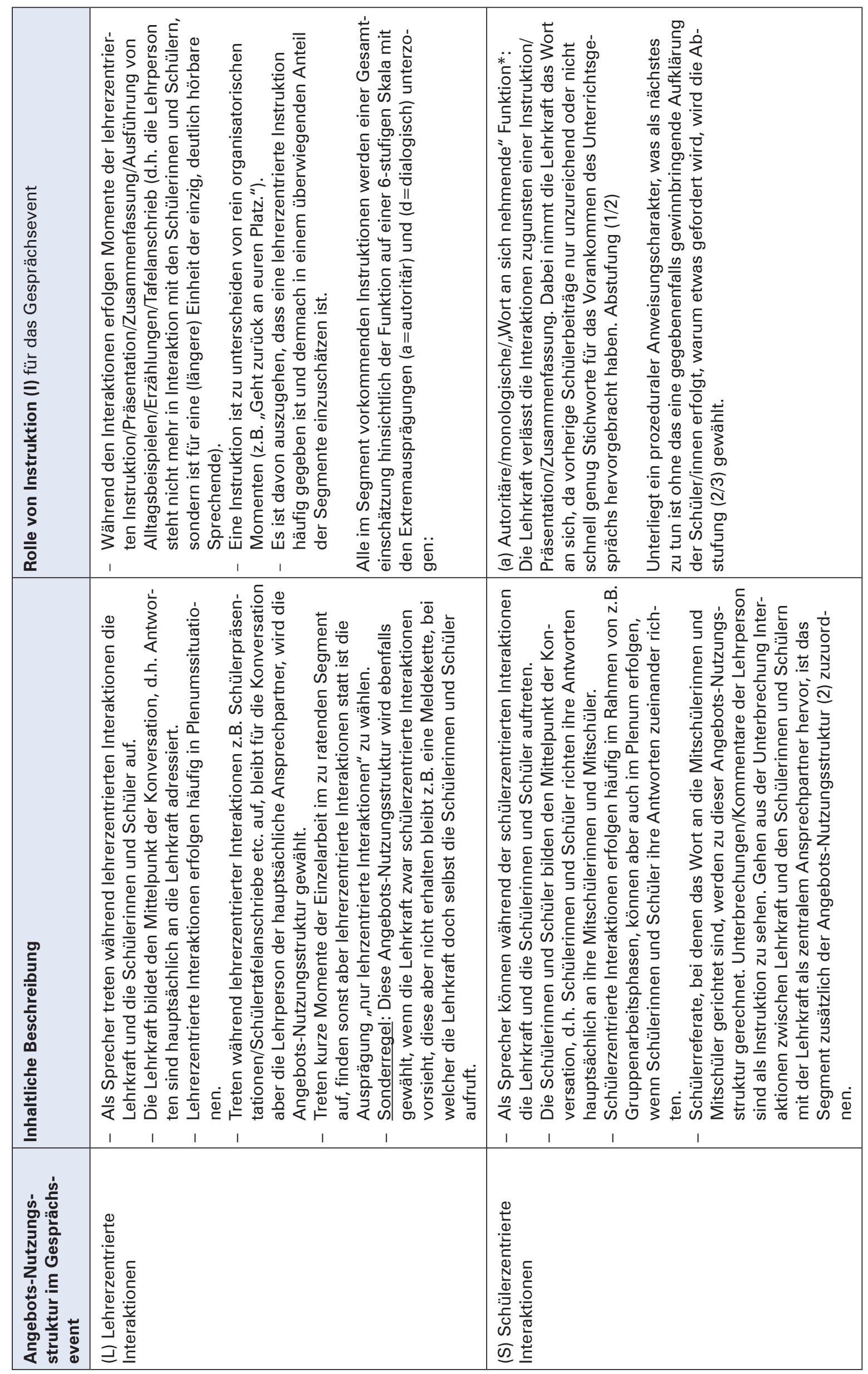




\begin{tabular}{|c|c|c|}
\hline \multicolumn{2}{|l|}{ 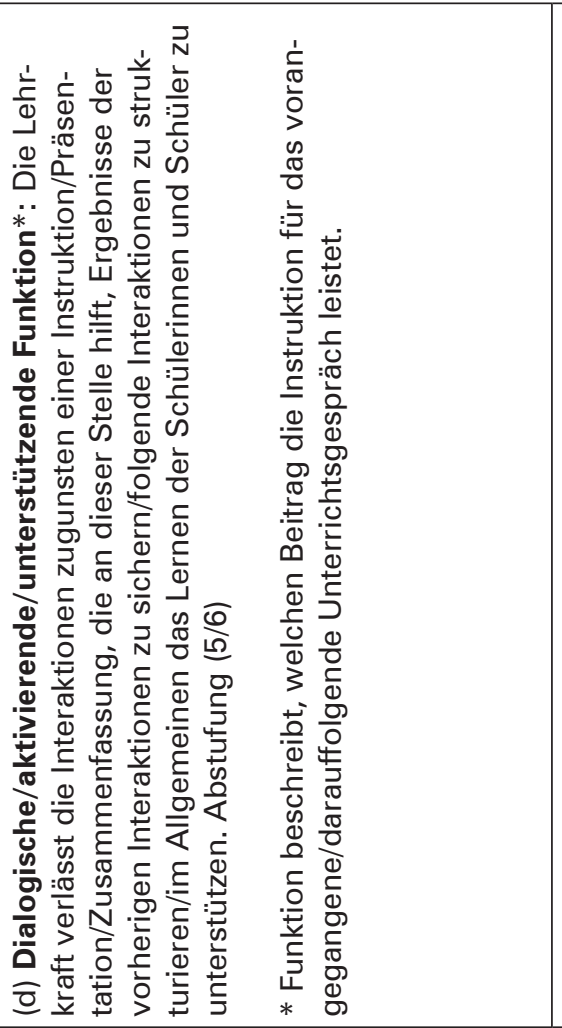 } & \\
\hline 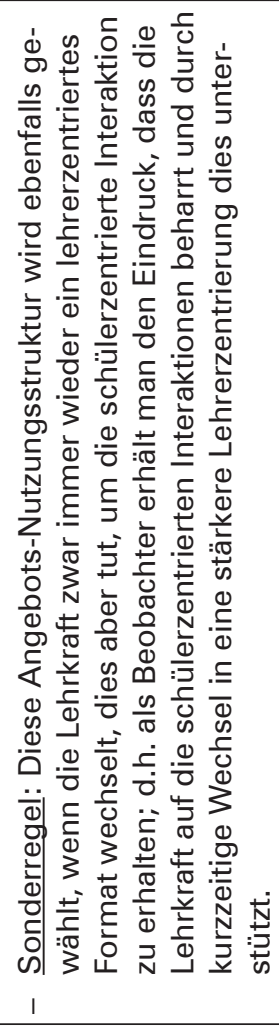 & 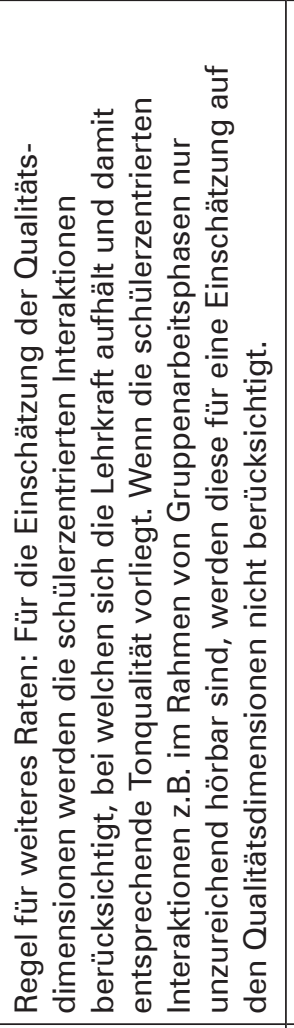 & 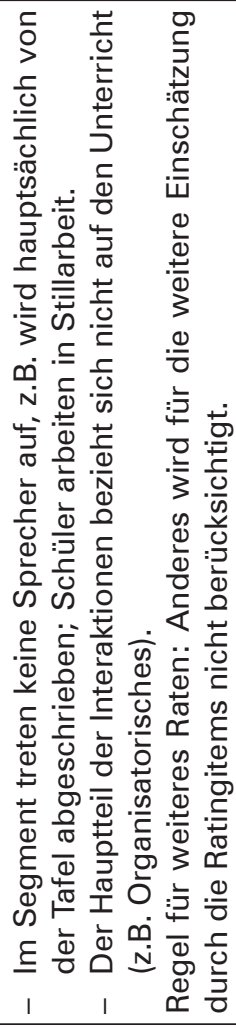 \\
\hline & & 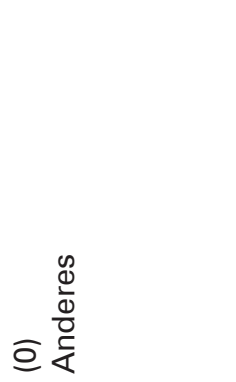 \\
\hline
\end{tabular}




\section{Beispielkodierung:}

\begin{tabular}{|l|l|l|l|}
\hline $10 \min$ & $10 \min$ & $10 \min$ & 10 min \\
\hline $\begin{array}{l}\text { (L) } \\
\text { (I) in Funktion a bis d }\end{array}$ & $\begin{array}{l}\text { (S+) } \\
\text { (I) in Funktion a bis d }\end{array}$ & $\begin{array}{l}\text { (L+) } \\
\text { (I) in Funktion a bis d }\end{array}$ & (L) \\
\hline
\end{tabular}

\section{Schritt 3: Kontextualisierung}

Für jedes 10 Minuten Segment wird eine Kontextualisierung bezüglich des (globalen) Zwecks des Segments vorgenommen, um später eine sequentielle (z.B. für Fallanalysen relevante) Beschreibung der Unterrichtsstunden vornehmen zu können. Mehrfachnennungen sind möglich. Dabei ist darauf zu achten, dass über eine längere Einheit der entsprechende Zweck beobachtbar ist (ein einmaliger Verweis der Lehrperson auf Inhalte aus der vorangegangenen Unterrichtsstunde reicht nicht aus, um „Vorwissensaktivierung“ zu kodieren). Zusätzlich wird am Ende der Sichtung der Stunde eine Gesamtkontextualisierung mit Blick auf die Lehr-Lernzielausrichtung der Stunde vorgenommen.

\begin{tabular}{|c|c|}
\hline Zweck & $\begin{array}{l}\text { - Vorwissensaktivierung/kognitive Aktivierung/Wiederholung: Bei der Vorwissensakti- } \\
\text { vierung wird das Wissen/Interessen, die Schüler/innen z.B. aus ihrem Alltag mitbringen, } \\
\text { erfragt/aktiviert. Alternativ/Zusätzlich werden Wissen/Inhalte aus vorherigen Unterrichts- } \\
\text { stunden/-einheiten/Schuljahren wiederholt, um eine Ausgangsbasis für den weiteren } \\
\text { Stundenverlauf zu legen. } \\
\text { - Strukturierung/Lernzielorientierung: Im Segment werden Maßnahmen unternommen, } \\
\text { die für die Strukturierung und/oder die Orientierung am Lernziel von Relevanz sind. } \\
\text { - Erarbeitung: Im Segment findet die Erarbeitung neuer Inhalte statt. Dies kann in Form ver- } \\
\text { schiedener Methoden, Gesprächsformen, Arbeitsaufträge etc. stattfinden. } \\
\text { - Ergebnissicherung: Im Segment werden erarbeitete Inhalte festgehalten und gesichert. } \\
\text { Dies kann in schriftlicher (z.B. Hefteintrag) oder mündlicher Form (z.B. Zusammenfassung) } \\
\text { geschehen. Eine Ergebnissicherung muss nicht zwangsläufig in einem Segment am Ende } \\
\text { einer Unterrichtsstunde erfolgen, sondern kann auch in Segmenten z.B. nach einer Schü- } \\
\text { lerarbeitsphase erfolgen. }\end{array}$ \\
\hline
\end{tabular}

\section{Beispielkodierung}

\section{Gesamtkontextualisierung}

Stundenthema:

Erkennbares Lernziel der Unterrichtsstunde:

\begin{tabular}{|l|l|l|l|}
\hline 10 min & 10 min & 10 min & 10 min \\
\hline $\begin{array}{l}\text { (L) } \\
\text { (I) in Funktion a bis d }\end{array}$ & $\begin{array}{l}\text { (S+) in Funktion a bis d } \\
\text { (I) }\end{array}$ & $\begin{array}{l}\text { (L }+) \\
\text { (I) in Funktion a bis d }\end{array}$ & (L) \\
\hline Zweck: Wiederholung & Zweck: Erarbeitung & Zweck: Erarbeitung & $\begin{array}{l}\text { Zweck: Ergebnissiche- } \\
\text { rung }\end{array}$ \\
\hline
\end{tabular}




\section{Schritt 4: Einschätzung 4x10 Minuten Segmente bezüglich der Sicht- und Tiefenstrukturen für jede der vier Qualitätsdimensionen}

Die vier Segmente werden hinsichtlich der Qualität ihrer Tiefenstrukturen entlang der vier Qualitätsdimensionen eingeschätzt.

Ratingregel 1: Für jedes Item werden zuerst die Handlungen der Schülerinnen und Schüler eingeschätzt, um zu erfassen, wie Routinen im Unterrichtgespräch bezüglich der Qualitätsdimensionen seitens der Schülerinnen und Schüler ausgeprägt sind (in Anlehnung an Reznitskaya et al., 2016).

Ratingregel 2: Die ratende Person orientiert sich im Manual an der Ausführung der Abstufung „adaptiv“, welche den höchst ausgeprägten Zustand und zugehörige Ankerbeispiele beschreibt.

Ratingregel 3: Zunächst wird eine Tendenzeinschätzung vorgenommen. Dabei stehen folgende Ausprägungen zur Verfügung:

\begin{tabular}{|c|c|}
\hline $\begin{array}{l}\text { Kein Nutzen/ } \\
\text { nicht vorhanden }\end{array}$ & $\begin{array}{l}\text { Eine Tendenzeinschätzung „Kein Nutzen“ wird gewählt, wenn eine zumeist au- } \\
\text { toritäre IRF Interpretation des Unterrichtsgesprächs vorliegt, eine Übersetzung } \\
\text { der Lehrziele in Lernziele für die Schülerinnen und Schüler fraglich ist oder zu } \\
\text { wenige Schülerbeteiligung vorliegt, um eine Einordnung vorzunehmen. }\end{array}$ \\
\hline Prozedurale Anwendung & $\begin{array}{l}\text { Diese Tendenzeinschätzung wird gewählt, wenn Aspekte der Qualitätsdimen- } \\
\text { sion beobachtbar sind, der Beobachter aber den Eindruck erhält, dass diese } \\
\text { stärker einer Abhandlung, um der Abhandlung Willen unterliegen (z.B. es wird } \\
\text { ein „Fahrplan“ aufgelegt ohne Rückversicherung, ob die Schüler/innen diesen } \\
\text { als Lernplan verstanden haben). Zusätzlich erhält man als Beobachter in Teilen } \\
\text { des zu ratenden Segments den Eindruck, dass eher der reibungsfreie Ablauf } \\
\text { des Skripts des Unterrichtsgesprächs zentral ist. Die Produktivität des Unter- } \\
\text { richtsgesprächs könnte an einigen Stellen noch gesteigert werden. Die Fein- } \\
\text { einschätzung (2) erfolgt, wenn die Abhandlung des Unterrichtsskripts von zen- } \\
\text { traler Rolle erscheint; Feineinschätzung (3) wenn zwar eine Skriptung vorliegt, } \\
\text { aber immer wieder Tendenzen von Adaptivität zu beobachten sind. }\end{array}$ \\
\hline Adaptive Anwendung & $\begin{array}{l}\text { Diese Tendenzeinschätzung wird gewählt, wenn Schülerinnen und Schüler } \\
\text { gemäß ihren Möglichkeiten agieren. D.h. wenn Schüler/innen z.B. Raum für } \\
\text { elaborierte Antworten haben und diese auch nutzen. Auf Seiten der Lehrkraft } \\
\text { bedeutet „adaptiv“, dass Aspekte nicht des Umsetzens willens geschehen, } \\
\text { sondern an den Bedarf der Schüler/innen angepasst sind (z.B. werden weitere } \\
\text { Erklärungen eingefordert, wenn diese für den Lernerfolg der Schüler/innen } \\
\text { bedeutsam sind). Der Lernerfolg stellt das zentrale Anliegen des Unterrichts- } \\
\text { gesprächs dar. Diese Ausprägung wird auch dann gewählt, wenn vereinzelt } \\
\text { weniger lernförderliche Interaktionen vorkommen (z.B. evaluatives Feedback } \\
\text { „Richtig“/„Falsch“), jedoch in dieser Situation für einen produktiven Gesprächs- } \\
\text { verlauf angemessen sind. Wenn eine bestimmte Angebotsstruktur seitens der } \\
\text { Lehrkraft nicht nötig ist (z.B. die Schüler/innen zur Beteiligung zu bestärken, } \\
\text { weil diese von sich aus eine hohe Bereitschaft zeigen), wird die Tendenzein- } \\
\text { schätzung „Adaptiv“ gewählt. }\end{array}$ \\
\hline
\end{tabular}

Ratingregel 4: Anschließend wird innerhalb der Tendenzeinschätzung eine Feineinschätzung vorgenommen $(0 / 1 ; 2 / 3 ; 4 / 5)$.

- Die Feineinschätzung 0 wird gewählt, wenn wesentliche Grundvoraussetzungen für das produktive Unterrichtsgespräch nicht gegeben sind wie z.B.: Schülerbeteiligung ist so unzureichend vorhanden, dass eine Einschätzung nicht möglich ist.

- Die höhere Feineinschätzung wird gewählt,

- wenn die Kriterien der Items im überwiegenden (zeitlichen) Anteil der Ratingsequenz beobachtet wurden.

- wenn der Beobachter den Eindruck erhält, dass „nur wenig fehlt“, um zu einer besseren Tendenzeinschätzung zu gelangen. 
- Für die Feinschätzung 2/3 gilt im Besonderen folgendes Kriterium:

- (2) eine Übersetzung gesetzter Lehrziele in eigene Lernziele scheint für die Schülerinnen und Schüler fraglich; die Abhandlung des prozeduralen Unterrichtsskripts steht im Fokus.

- (3) eine Übersetzung gesetzter Lehrziele in eigene Lernziele scheint für die Schülerinnen und Schüler an einigen Stellen möglich; im prozeduralen Unterrichtsskript gibt es adaptive Tendenzen.

\section{Beispielkodierung:}

\section{Gesamtkontextualisierung}

Stundenthema:

Erkennbares Lernziel der Unterrichtsstunde:

\begin{tabular}{|c|c|c|c|}
\hline $10 \mathrm{~min}$ & $10 \mathrm{~min}$ & $10 \mathrm{~min}$ & $10 \mathrm{~min}$ \\
\hline $\begin{array}{l}\text { (L) } \\
\text { (I) in Funktion a bis d }\end{array}$ & $\begin{array}{l}(\mathrm{S}+) \\
\text { (I) in Funktion a bis d }\end{array}$ & $\begin{array}{l}(L+) \\
\text { (I) in Funktion a bis d }\end{array}$ & $(\mathrm{L})$ \\
\hline Zweck: Wiederholung & Zweck: Erarbeitung & Zweck: Erarbeitung & $\begin{array}{l}\text { Zweck: } \\
\text { Ergebnissicherung }\end{array}$ \\
\hline $\begin{array}{l}\text { Sichtstruktur: } \\
\text { Einschätzungen } \\
\text { aller vorkommender } \\
\text { Methoden } \\
\text { Tiefenstrukturen: \#1-\#4 }\end{array}$ & $\begin{array}{l}\text { Sichtstruktur: } \\
\text { Einschätzungen } \\
\text { aller vorkommender } \\
\text { Methoden } \\
\text { Tiefenstrukturen: \#1-\#4 }\end{array}$ & $\begin{array}{l}\text { Sichtstruktur: } \\
\text { Einschätzungen } \\
\text { aller vorkommender } \\
\text { Methoden } \\
\text { Tiefenstrukturen: \#1-\#4 }\end{array}$ & $\begin{array}{l}\text { Sichtstruktur: } \\
\text { Einschätzungen } \\
\text { aller vorkommender } \\
\text { Methoden } \\
\text { Tiefenstrukturen: \#1-\#4 }\end{array}$ \\
\hline
\end{tabular}




\section{Sichtstrukturebene: Methoden zur Unterstützung/Anregung/Erhaltung des Unterrichtsgesprächs}

Für jede im Segment vorkommende Methode sind folgende Einschätzungen vorzunehmen. Geht eine Methode über Segmentgrenzen hinweg, ist die Methode im Folgesegment nicht nochmal zu wählen, um später eine korrekte Quantifizierung vornehmen zu können. Die Einschätzung der Qualität erfolgt für die Methode über Segmentgrenzen hinweg, d.h. ggf. muss die Einschätzung nachkorrigiert werden, sollte sich die Qualitätseinschätzung im Folgesegment ändern. Wird die gleiche Methode in einem Folgesegment nochmal durchgeführt (z.B. eine zweite Gruppenarbeit), ist diese neu und unabhängig von der vorherigen Methode zu erfassen.

Benennen der $\quad$ - Fahrplan (\#1): Ein Fahrplan listet für die Schüler/innen den Ablauf des Unterrichts. Dies Methode kann an der Tafel, einer PPT-Folie, einer Overheadfolie etc. geschehen.

- Ausgehändigter Lernplan (\#1): Ein Lernplan wird an die Schüler/innen ausgehändigt. Ggf. enthält er individuelle Dokumentationsmöglichkeiten des eigenen Lernfortschritts.

- Stiller Impuls (\#2): Bei einem Stillen Impuls führt die Lehrkraft ohne weiteres Kommentieren etwas vor, zeigt eine Abbildung auf etc. Der Impuls soll die Schüler/innen im Folgendem zu einem Gespräch anregen.

- Medialer Impuls (\#2): Bei einem medialen Impuls kommt ein Film/ein Hörbespiel etc. zum Einsatz, welches im Folgenden zu einem Gespräch anregen kann. PPT-Folien oder Tafelanschriebe zählen dabei nicht als Impuls, sondern werden in der Kontextualisierung als mediale Unterstützung betrachtet.

- Lehrerexperiment/-demonstration (\#2): Bei einem Lehrerexperiment/-demonstration führt die Lehrkraft ein Experiment, eine Prozedur etc. vor, welche im Folgenden zu einem Gespräch anregen können. PPT-Folien, Tafelanschriebe zählen dabei nicht als Impuls, sondern werden in der Kontextualisierung als mediale Unterstützung betrachtet.

- Schülerpräsentationen und -tafelanschriebe (\#2): Schüler/innen erhalten die Möglichkeit für einen Tafelanschrieb, eine Präsentation oder ein Referat. Die Präsentation muss über einen längeren Zeitraum gehen und ist abzugrenzen von Kurzevents wie z.B. Lösungen auf einer Folie eintragen.

- Schülerexperiment (\#2): Ein/e Schüler/in führt ein Experiment vor der Klasse vor.

- Motorische Lernaktivitäten/Rollen- und Modellspiele (\#2): Inhalte/Abläufe werden mithilfe von körperlichen Bewegungsspielen visualisiert (z.B. Betrunkenheitszustand durch das Aufsetzen entsprechender Brillen).

- Klassische Gruppenarbeit (\#2): Eine klassische Gruppenarbeit besteht aus einem Arbeitsauftrag für die Schüler/innen in Zusammenarbeit mit einer Gruppe. Nach der Erarbeitung werden Ergebnisse im Plenum präsentiert.

- Gruppenpuzzle (\#2/\#3): Im Rahmen des Gruppenpuzzles arbeiten die Schüler/innen zunächst in Expertengruppen an einem gezielten Themenbereich, um dann in Stammgruppen ihre Mitschüler/innen darüber zu informieren.

- Think-Pair-Share (\#2/\#3): Nach einer kurzen individuellen Nachdenkzeit (Think), tauschen sich die Schüler/innen mit einem weiteren Mitschüler/in (zumeist dem Nachbarn) über den Lerninhalt aus (Pair), um diesen anschließend im Plenum vorzustellen (Share).

- Four Corners/Speakers Corner (\#2/\#3): Ein/e Schüler/in stellt sich auf eine im Klassenzimmer gut sichtbare Position, z.B. Stuhl, Ecke o.ä. und gibt seine/ihre Position/ Meinung kund (Speakers Corner). Alternativ wird die Klasse aufgefordert, sich entlang ihrer Meinungen/Lösungen etc. in die vier Ecken des Klassenzimmers zu verteilen (Four Corners).

- Methoden des Meet and Greet (\#2/\#3): Die Schüler/innen überlegen sich Aufgaben und dazugehörige Lösungen: Auf einen Zettel wird auf der Vorderseite die Aufgabe gestellt und auf der Rückseite die Lösung notiert. Die Schüler/innen gehen mit ihren Zetteln durch die Klasse und bearbeiten gegenseitig die Aufgaben.

- Sitzkreis (\#2/\#3): Schüler/innen und Lehrkraft diskutieren im Stuhlkreis, sodass alle Teilnehmer Blickkontakt zueinander haben. Diese Methode wird auch gewählt, wenn die Sitzordnung im Klassenzimmer dauerhaft so verändert wurde, dass die Schüler/innen Blickkontakt zueinander haben (z.B. U-Form).

- Metaplanmethode/„Write to support Talk“ (\#2/\#3): Schüler/innen notieren Vorwissen, Interessen, Vorschläge, etc. auf Karteikarten/in ihrem Heft. Anschließend werden diese an die Tafel gepinnt, vorgestellt und gemeinsam geordnet. Alternativ können die verschriftlichten Gedanken auch mündlich besprochen werden.

- Brainstorming (\#2/\#3): Einstiegsmethode, bei der sich Schüler/innen frei zu einem Thema äußern.

- Blitzlicht (\#2/\#3): Jede/r Schüler/in gibt kurz ihre/seine Meinung zu einem Thema ab. Die Lehrperson erhält somit ein gutes Meinungs-/Stimmungsbild. 


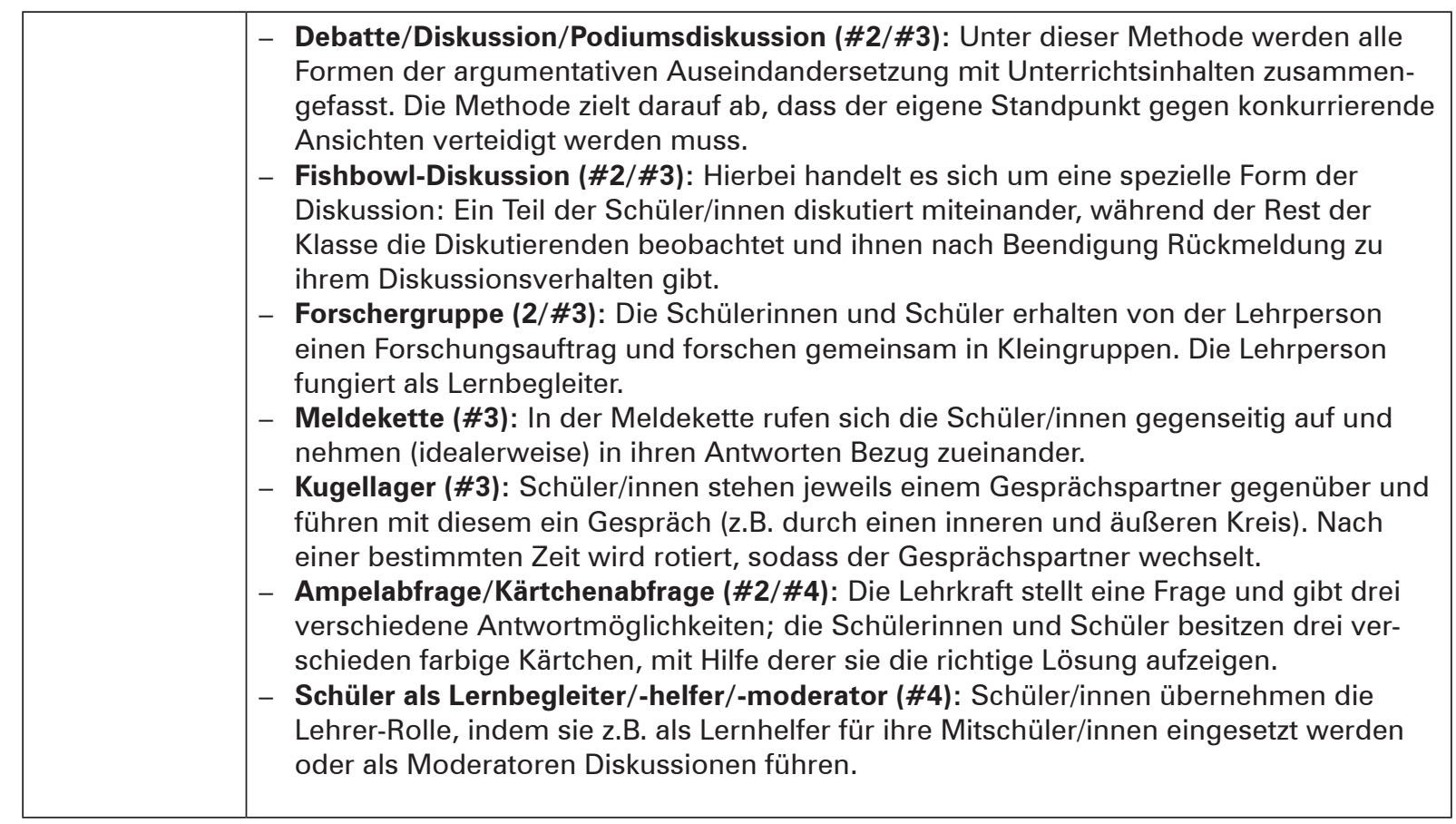

\begin{tabular}{|c|c|c|c|c|c|}
\hline \multicolumn{2}{|c|}{ Kein Nutzen } & \multicolumn{2}{|c|}{ Prozedurale Anwendung } & \multicolumn{2}{|c|}{ Adaptive Anwendung } \\
\hline 0 & 1 & 2 & 3 & 4 & 5 \\
\hline \multicolumn{2}{|c|}{$\begin{array}{l}\text { Für die eingesetzte Methode } \\
\text { ist kein Nutzen für das Unter- } \\
\text { richtsgespräch erkennbar. } \\
\text { - Die Feineinstufung } 1 \text { ist zu } \\
\text { wählen, wenn die Methode } \\
\text { lediglich eine visualisieren- } \\
\text { de Funktion hat (z.B. ein } \\
\text { Lehrerexperiment wird zu } \\
\text { Visualisierungszwecken } \\
\text { durchgeführt; spielt aber } \\
\text { für das Unterrichtsge- } \\
\text { spräch keine wesentliche } \\
\text { Rolle) }\end{array}$} & \multicolumn{2}{|c|}{$\begin{array}{l}\text { Die Lehrkraft transferiert eine } \\
\text { Methode zum produktiven } \\
\text { Unterrichtsgespräch und } \\
\text { handelt diese prozedural ab. } \\
\text { Prozedura/ bedeutet dabei } \\
\text { - die Methode erfährt keine } \\
\text { Adaption an den Bedarf } \\
\text { der Klasse } \\
\text { - für die Methode ist kein } \\
\text { Bedarf erkennbar/Methode } \\
\text { wird um der Methode Wil- } \\
\text { len eingesetzt } \\
\text { - die Methode unterstützt } \\
\text { das Unterrichtsgespräch } \\
\text { nicht notwendigerweise }\end{array}$} & \multicolumn{2}{|c|}{$\begin{array}{l}\text { Die Lehrkraft setzt eine er- } \\
\text { lernte oder eigene Methode } \\
\text { adaptiv ein } \\
\text { Adaptiv bedeutet dabei } \\
\text { - } \text { die Methode führt im Fol- } \\
\text { genden zu einem produkti- } \\
\text { ven Unterrichtsgespräch } \\
\text { - } \text { durch die Methode wird er- } \\
\text { reicht, dass viele Schüler/ } \\
\text { innen aktiv in das Unter- } \\
\text { richtsgespräch eingebun- } \\
\text { den werden } \\
\text { - } \text { die Methode erscheint im } \\
\text { Schwierigkeitsgrad/Dauer/ } \\
\text { Instruktion an den Bedarf } \\
\text { der Klasse angepasst } \\
\text { - } \text { die Methode wirkt auf den } \\
\text { Kontext angepasst } \\
\text { - } \text { als Beobachter erhält man } \\
\text { den Eindruck, die Methode } \\
\text { wurde nicht um der Me- } \\
\text { thode Willen eingesetzt, } \\
\text { sondern um die Qualität } \\
\text { des Unterrichtsgesprächs } \\
\text { gezielt zu unterstützen }\end{array}$} \\
\hline
\end{tabular}




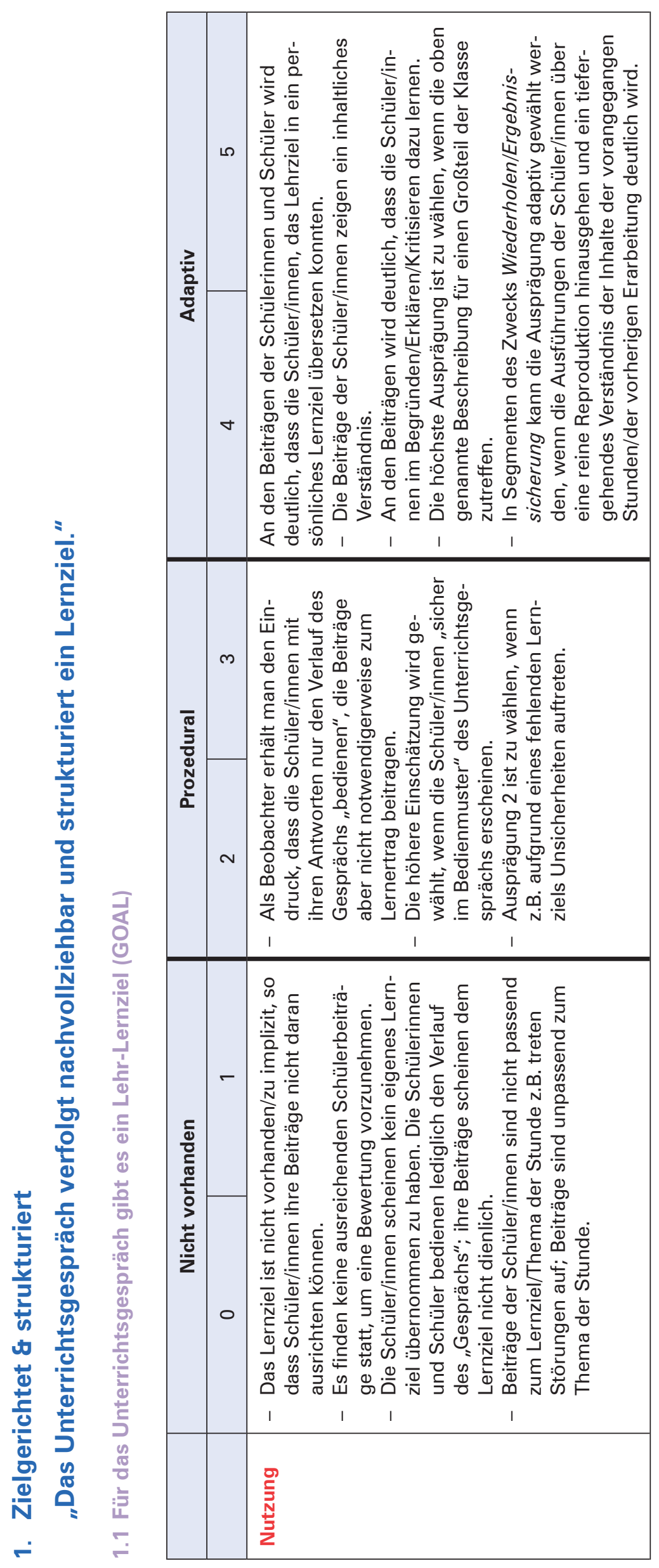




\begin{tabular}{|c|c|c|c|}
\hline \multicolumn{2}{|c|}{ 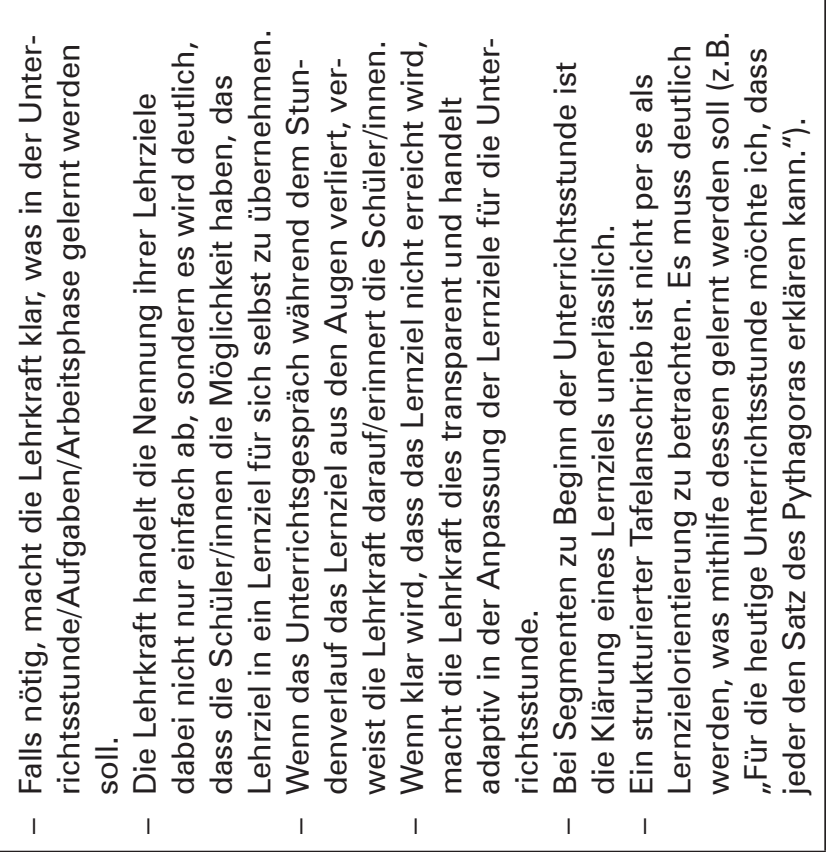 } & 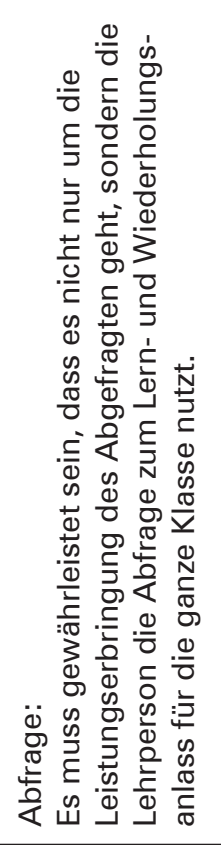 & 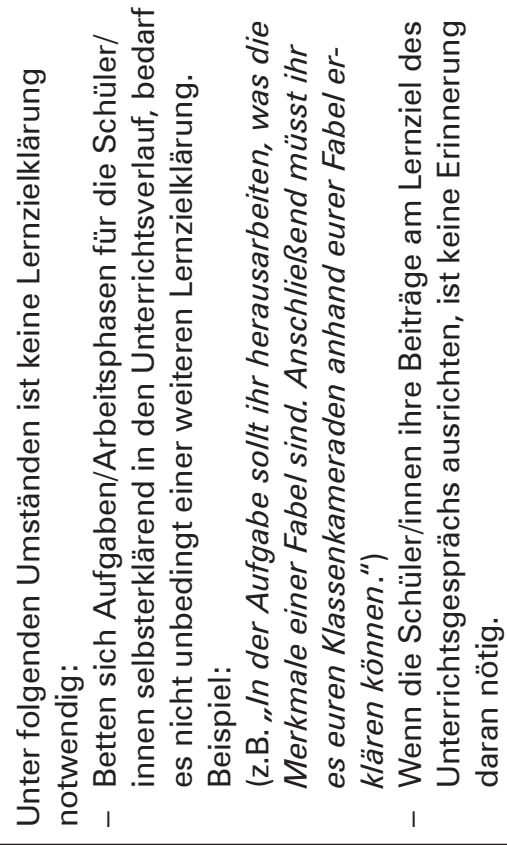 \\
\hline \multicolumn{2}{|c|}{ 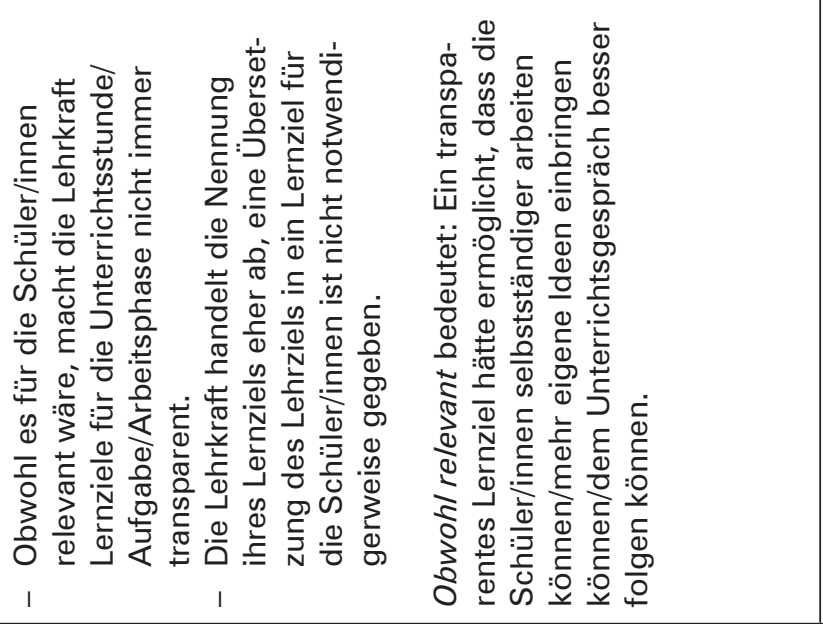 } & & \\
\hline \multicolumn{2}{|l|}{ 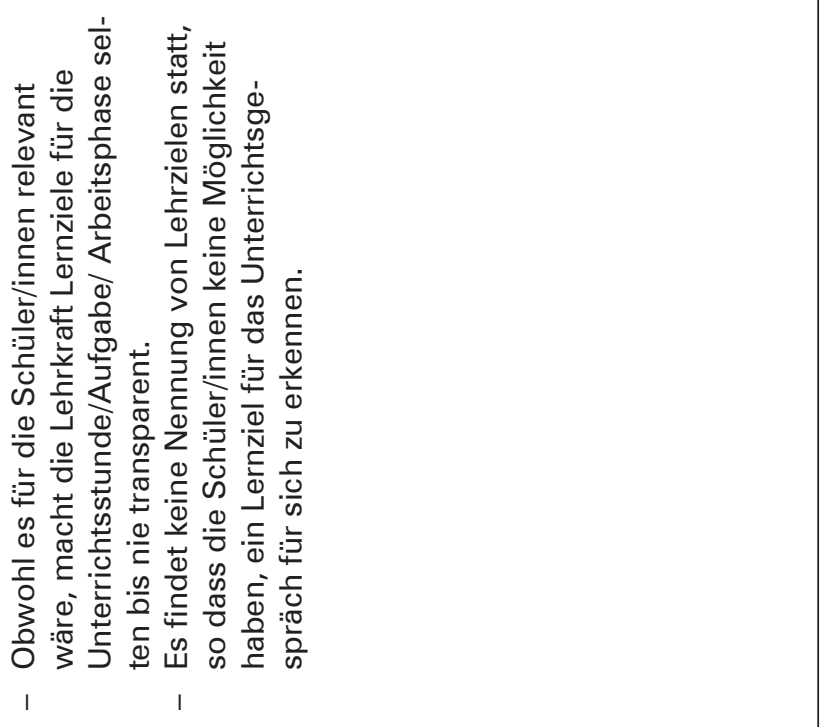 } & & \\
\hline & & & \\
\hline
\end{tabular}




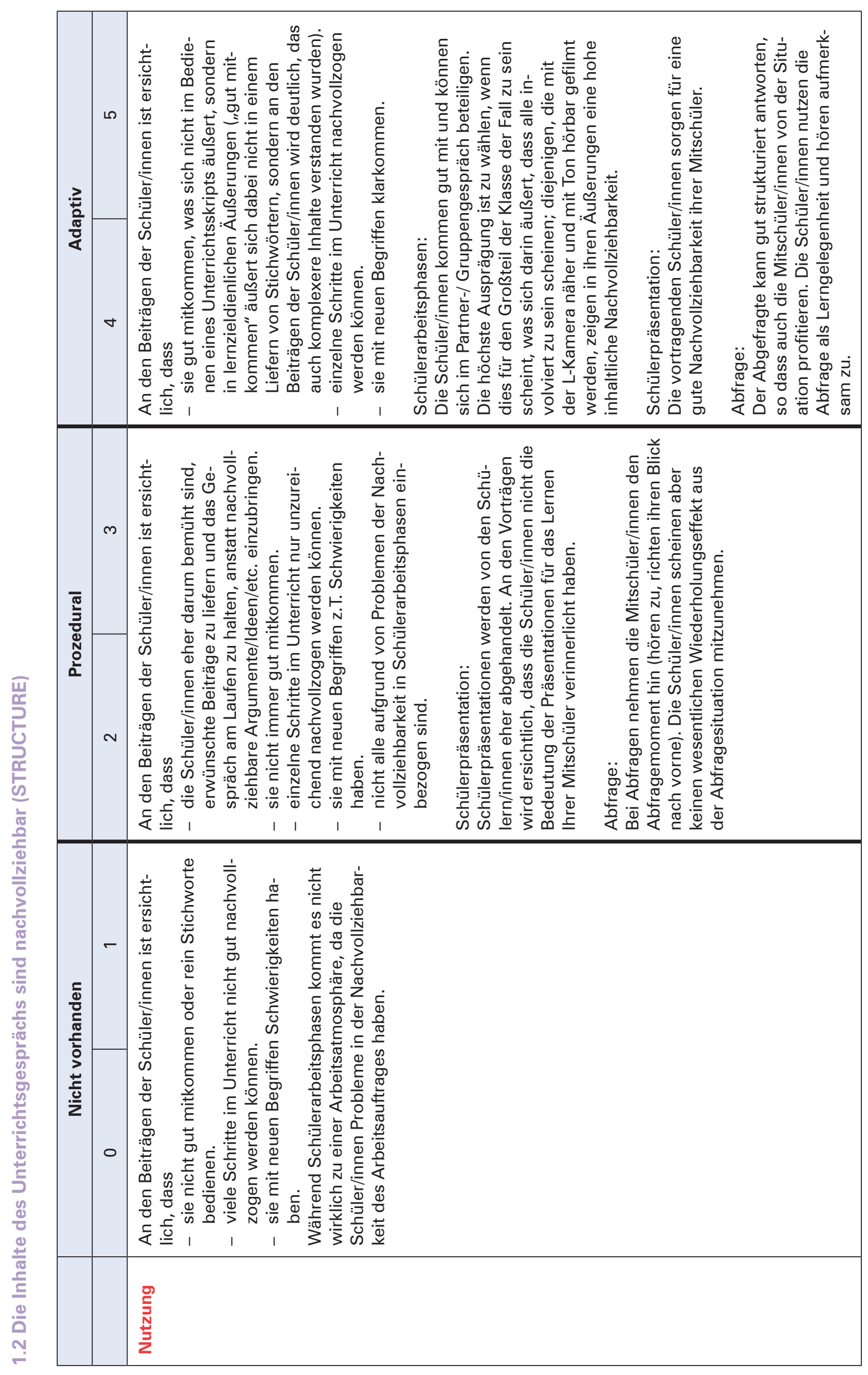




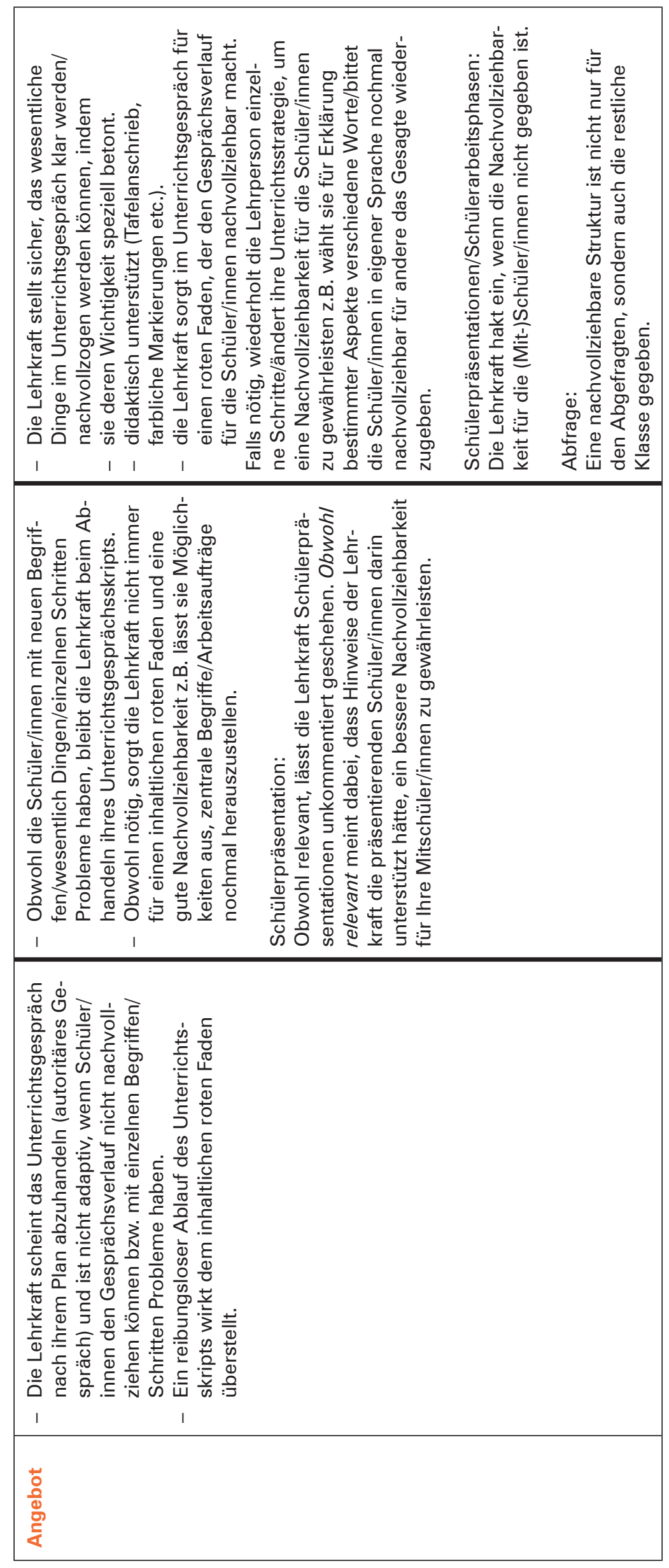




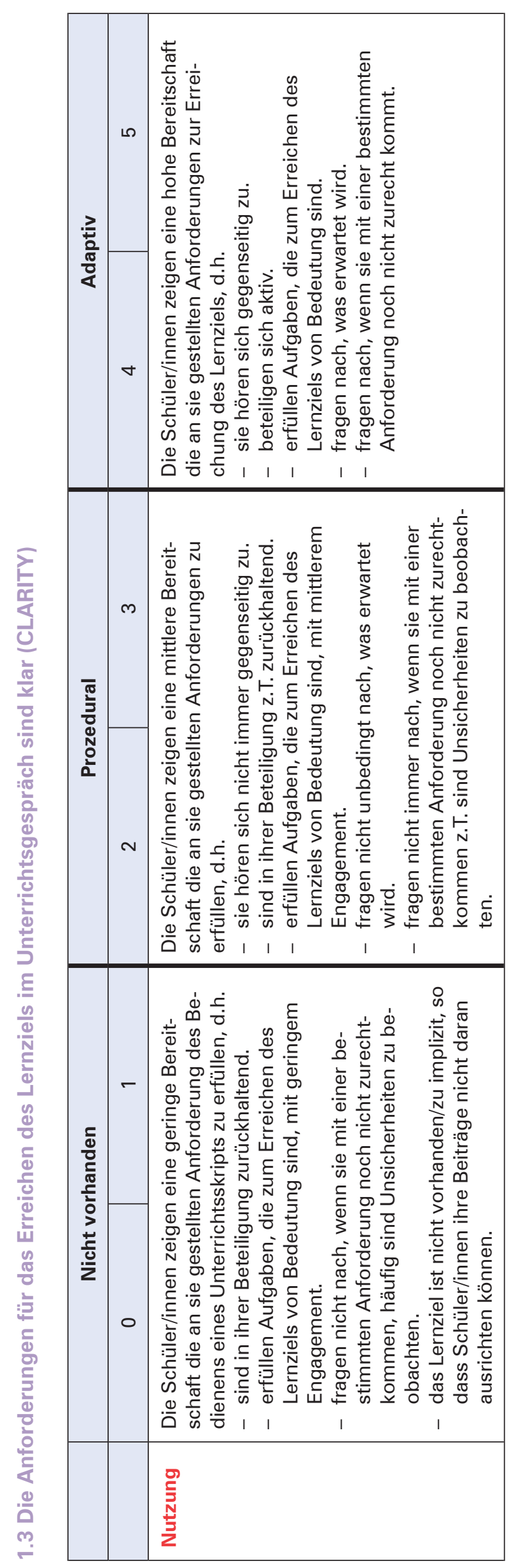




\begin{tabular}{|c|c|c|c|}
\hline 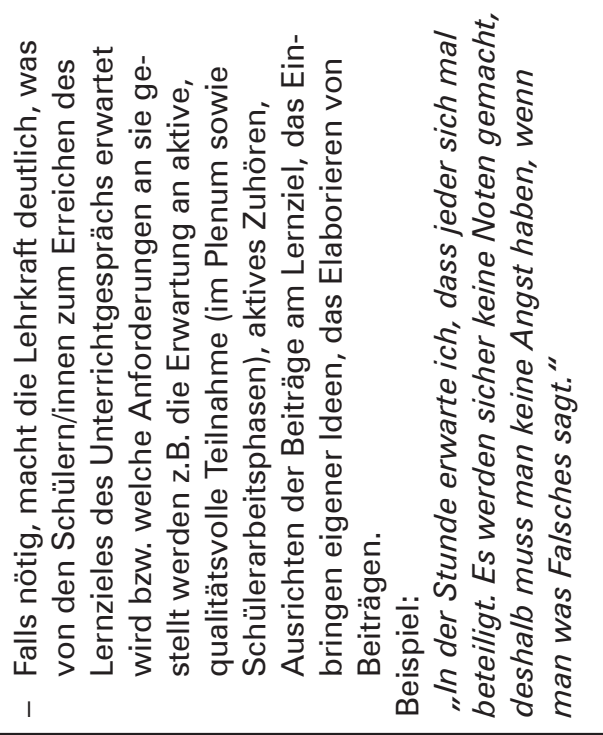 & 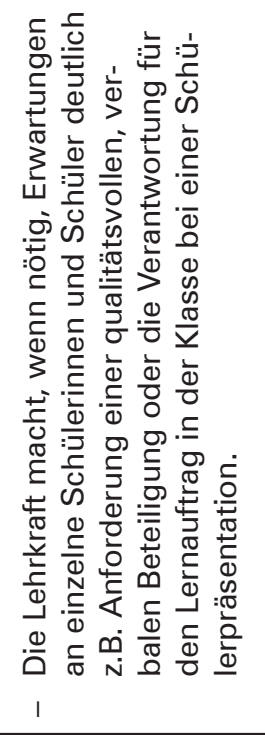 & 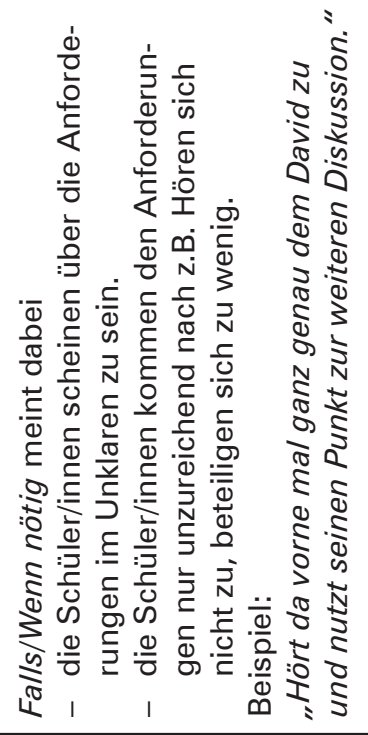 & 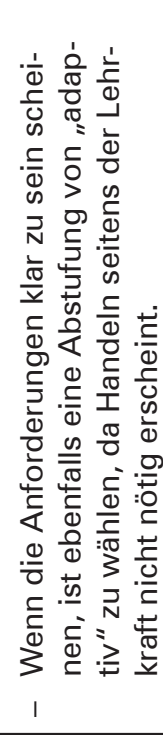 \\
\hline 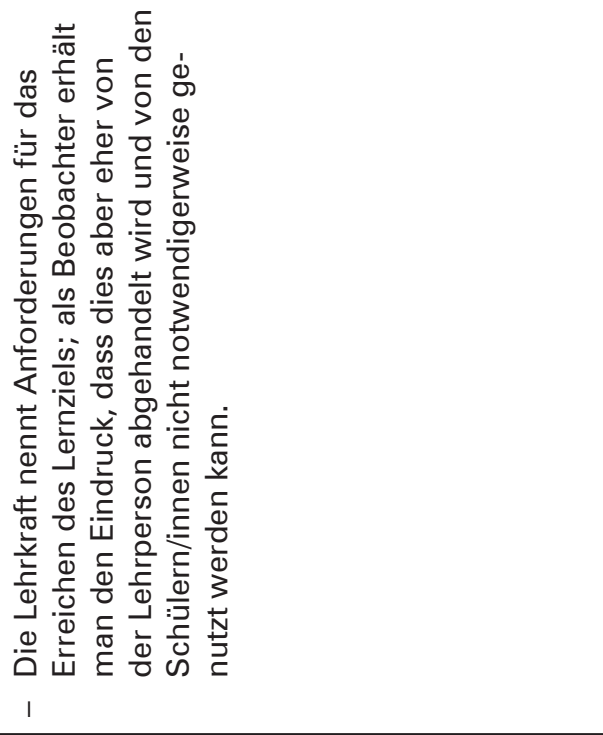 & & & \\
\hline 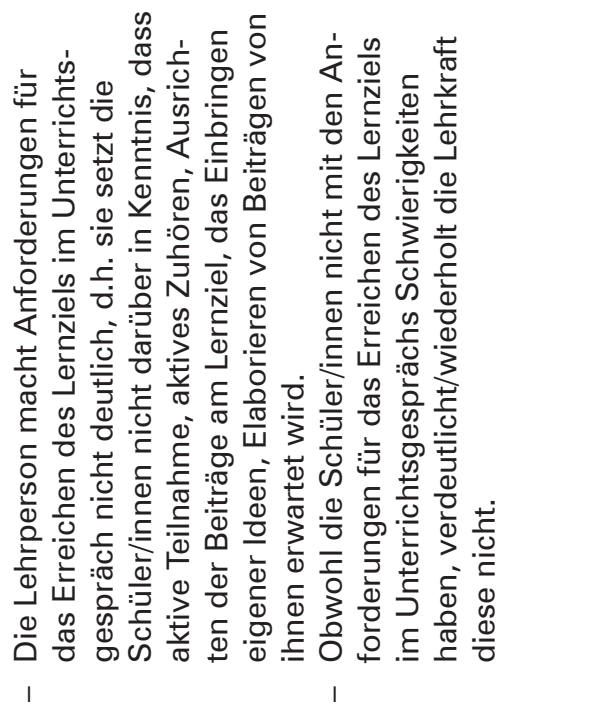 & & & \\
\hline & & & \\
\hline
\end{tabular}



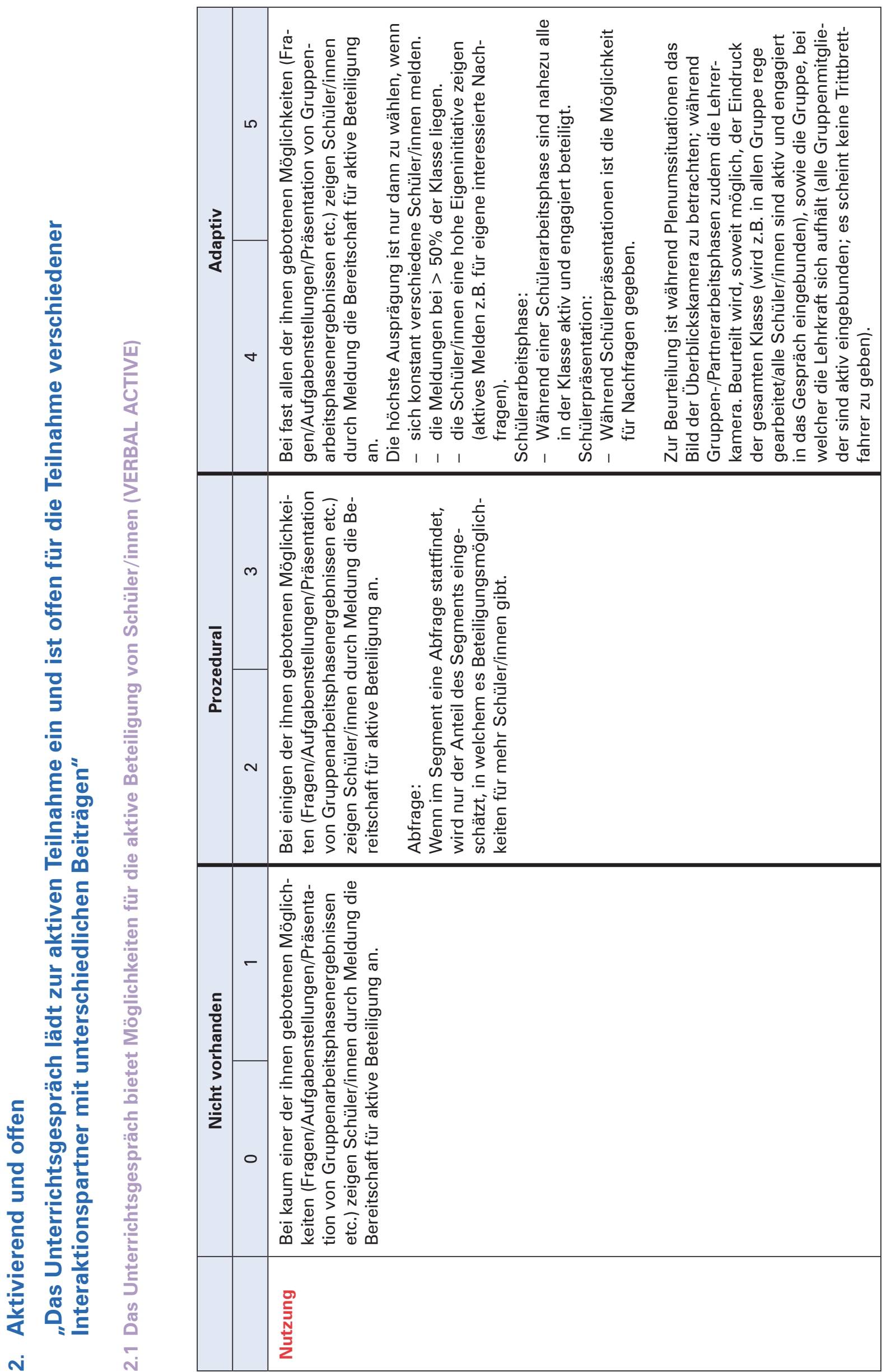


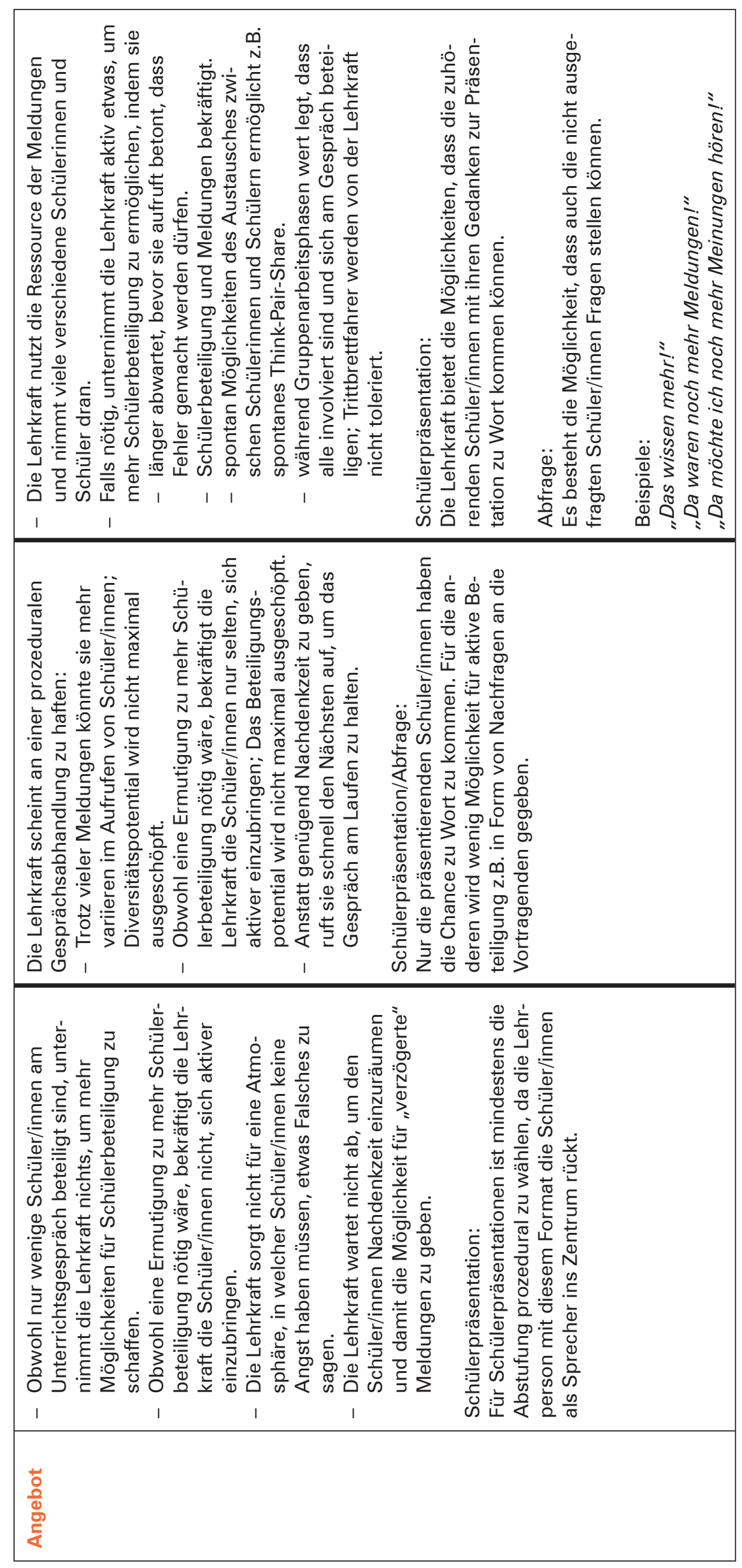




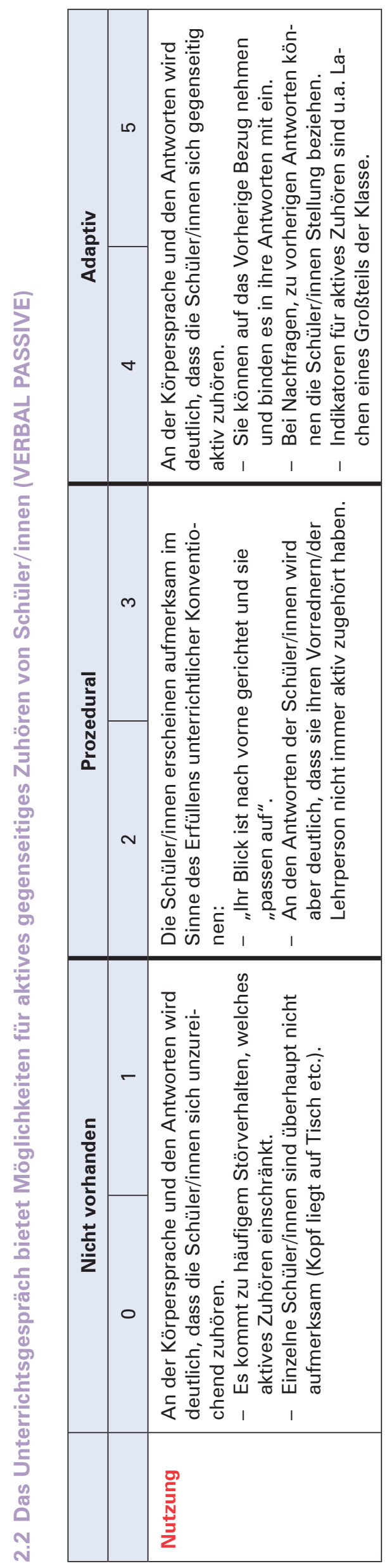




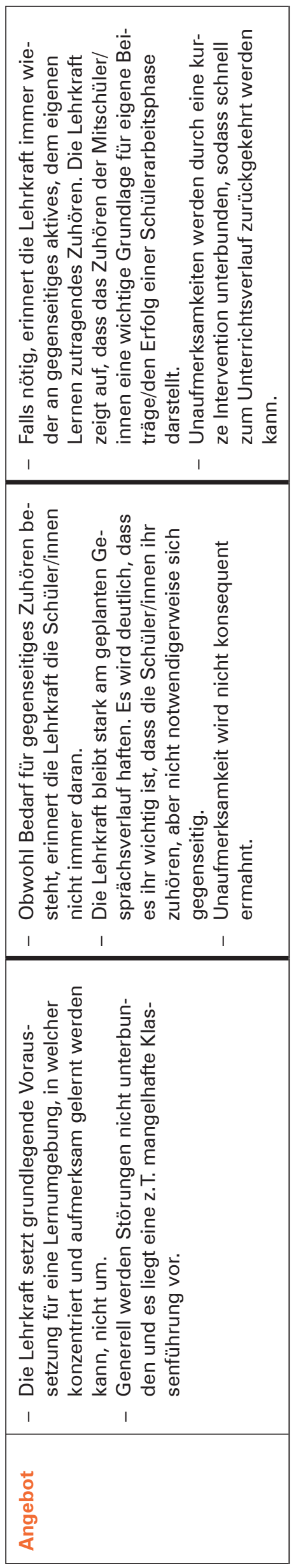




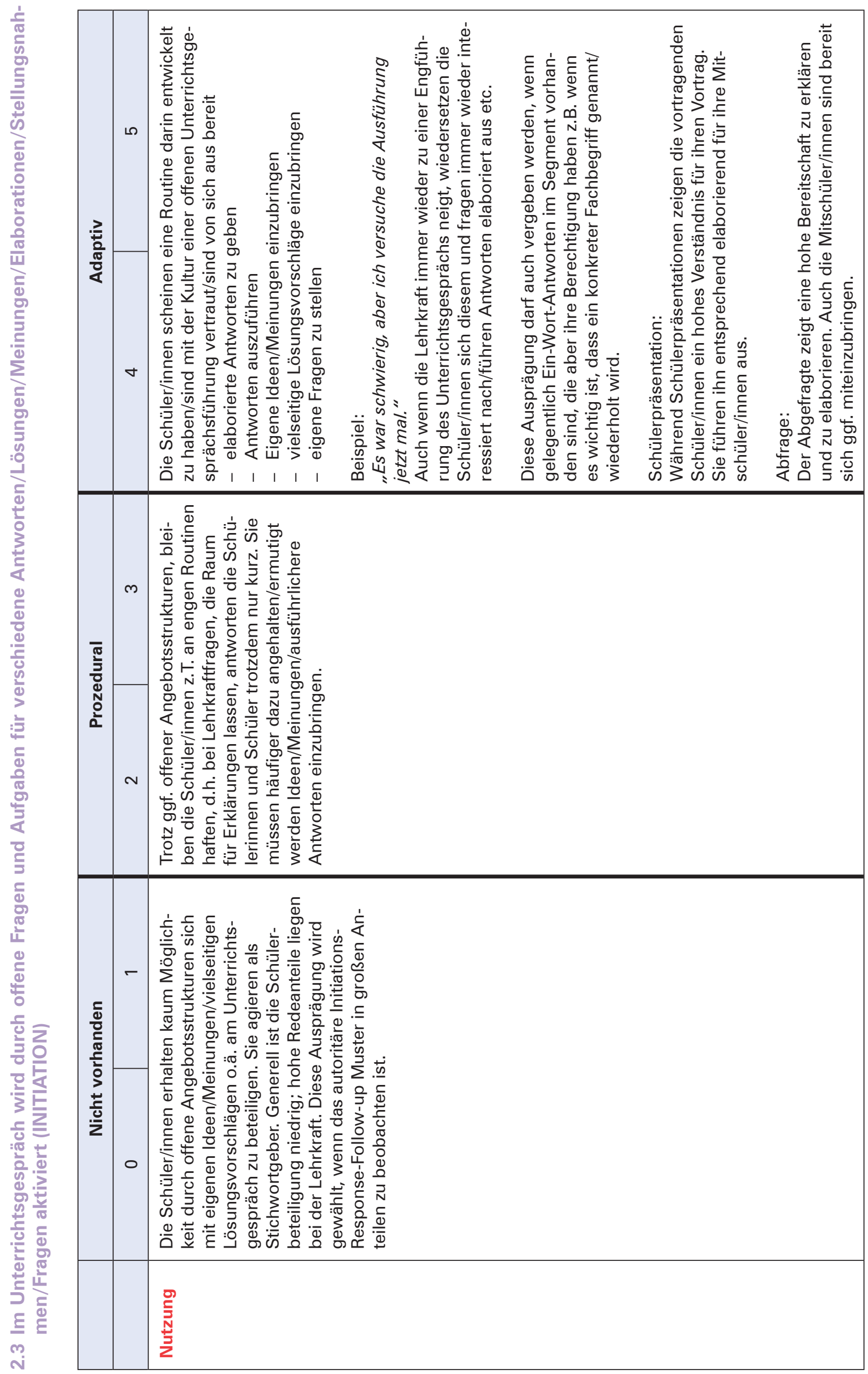




\begin{tabular}{|c|c|c|c|c|}
\hline 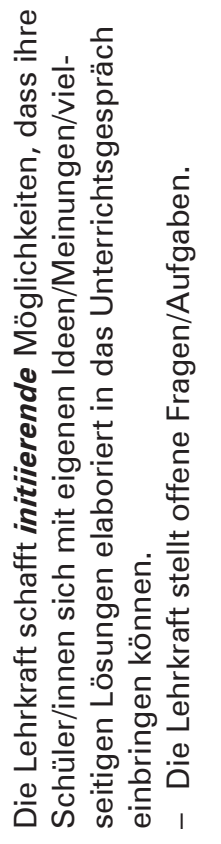 & 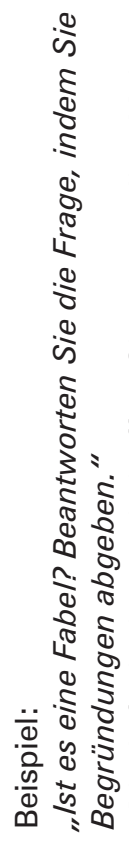 & 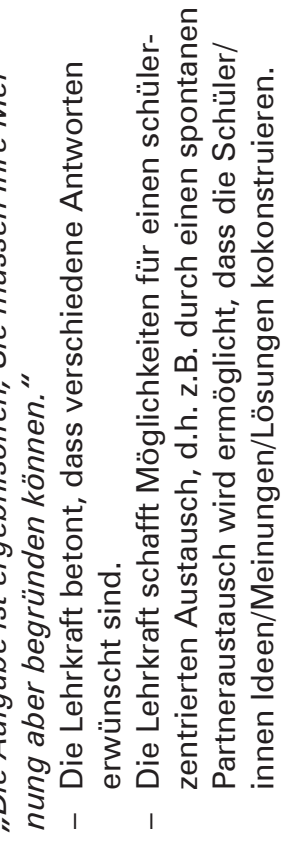 & 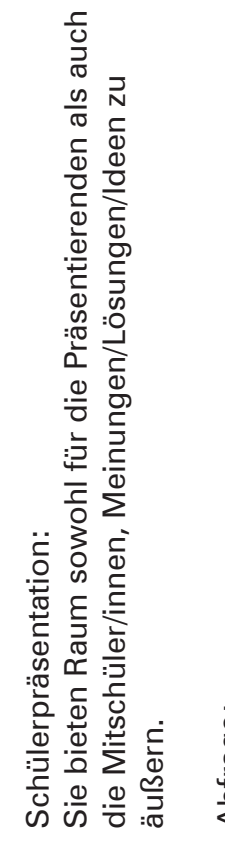 & 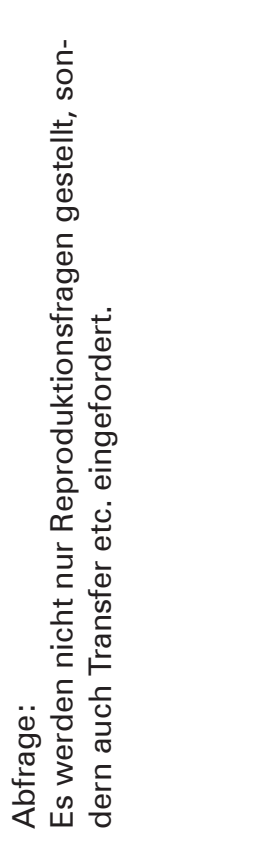 \\
\hline 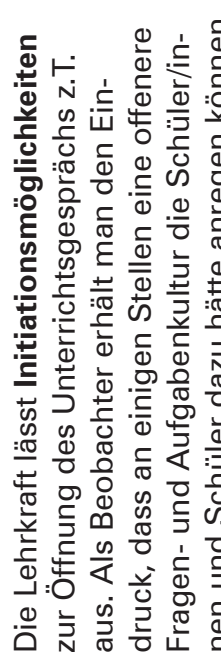 & 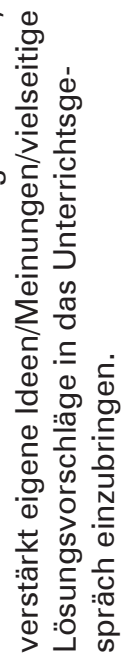 & 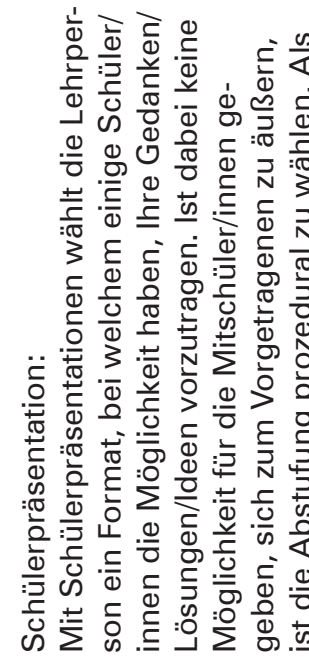 & 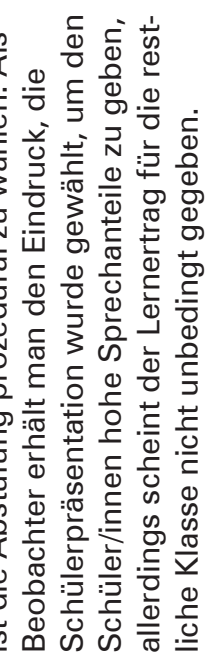 & 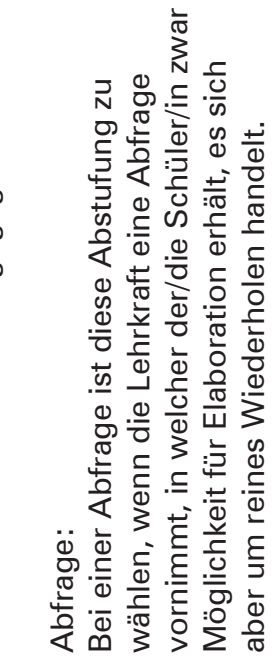 \\
\hline \multicolumn{5}{|c|}{ 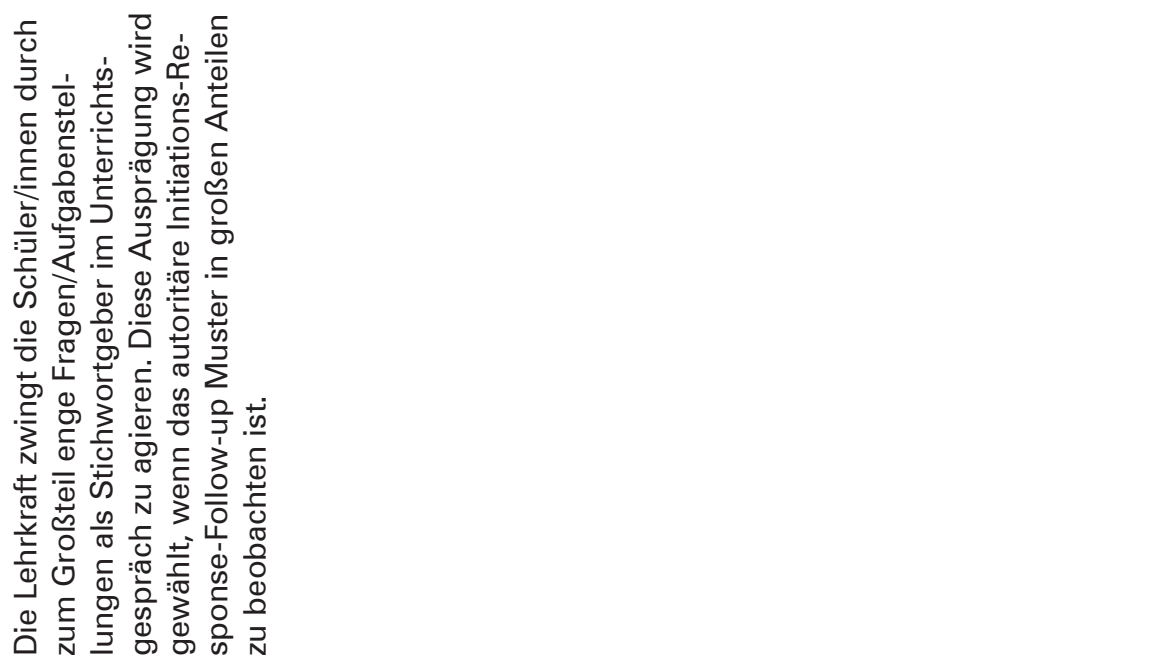 } \\
\hline 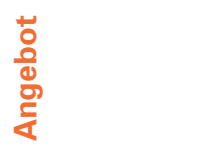 & & & & \\
\hline
\end{tabular}




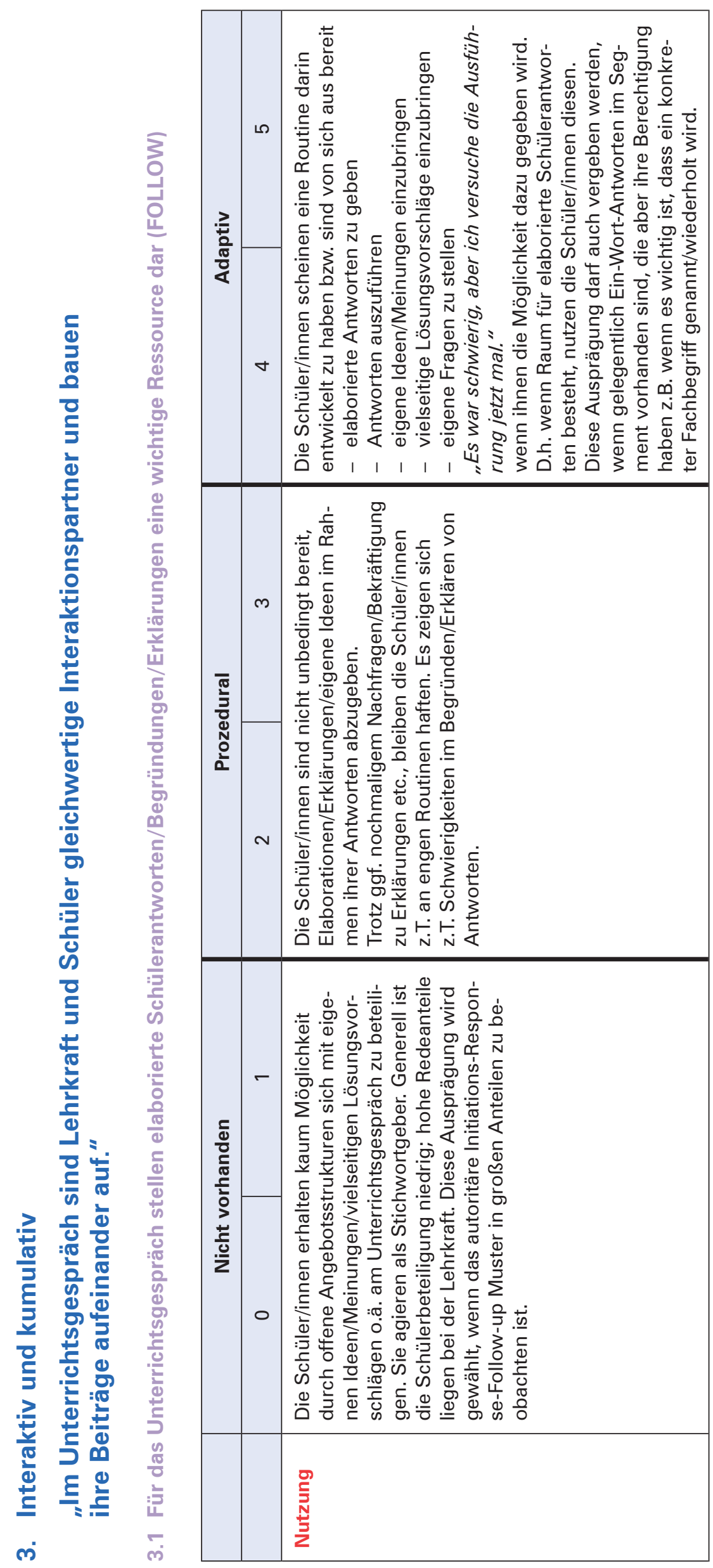




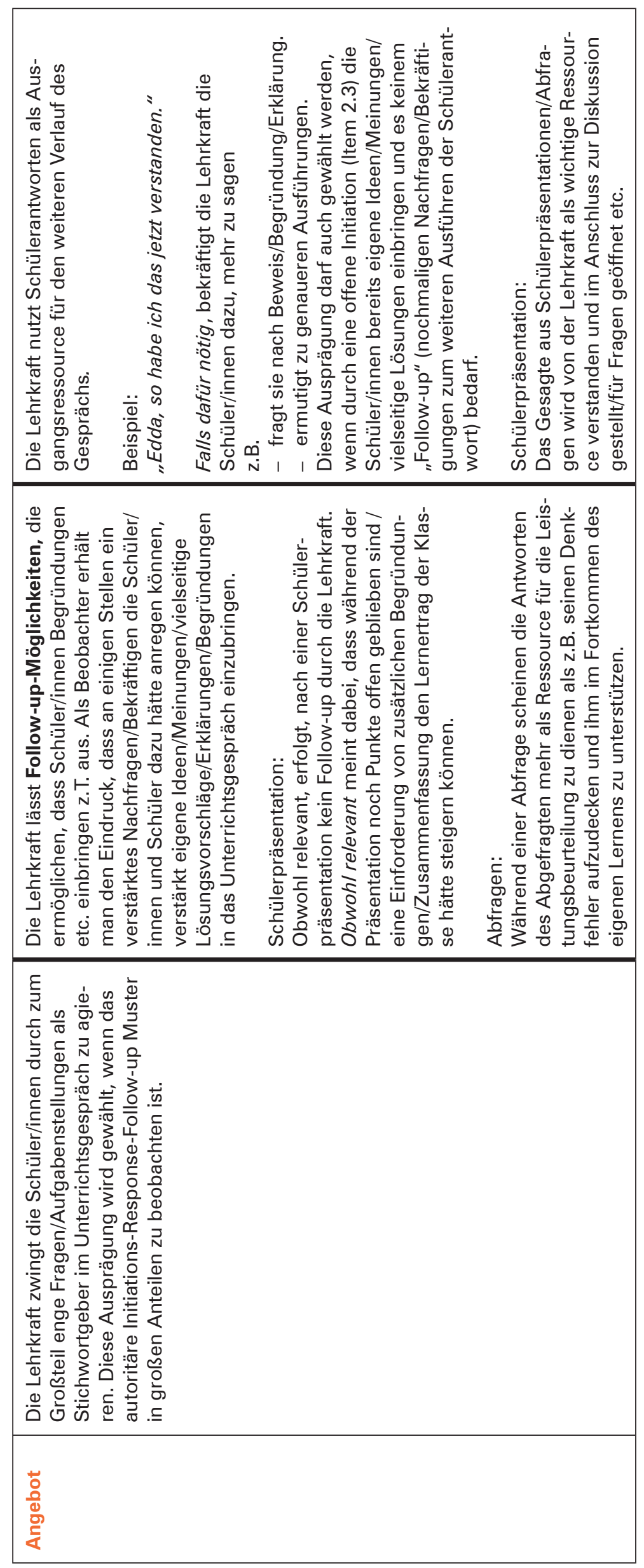




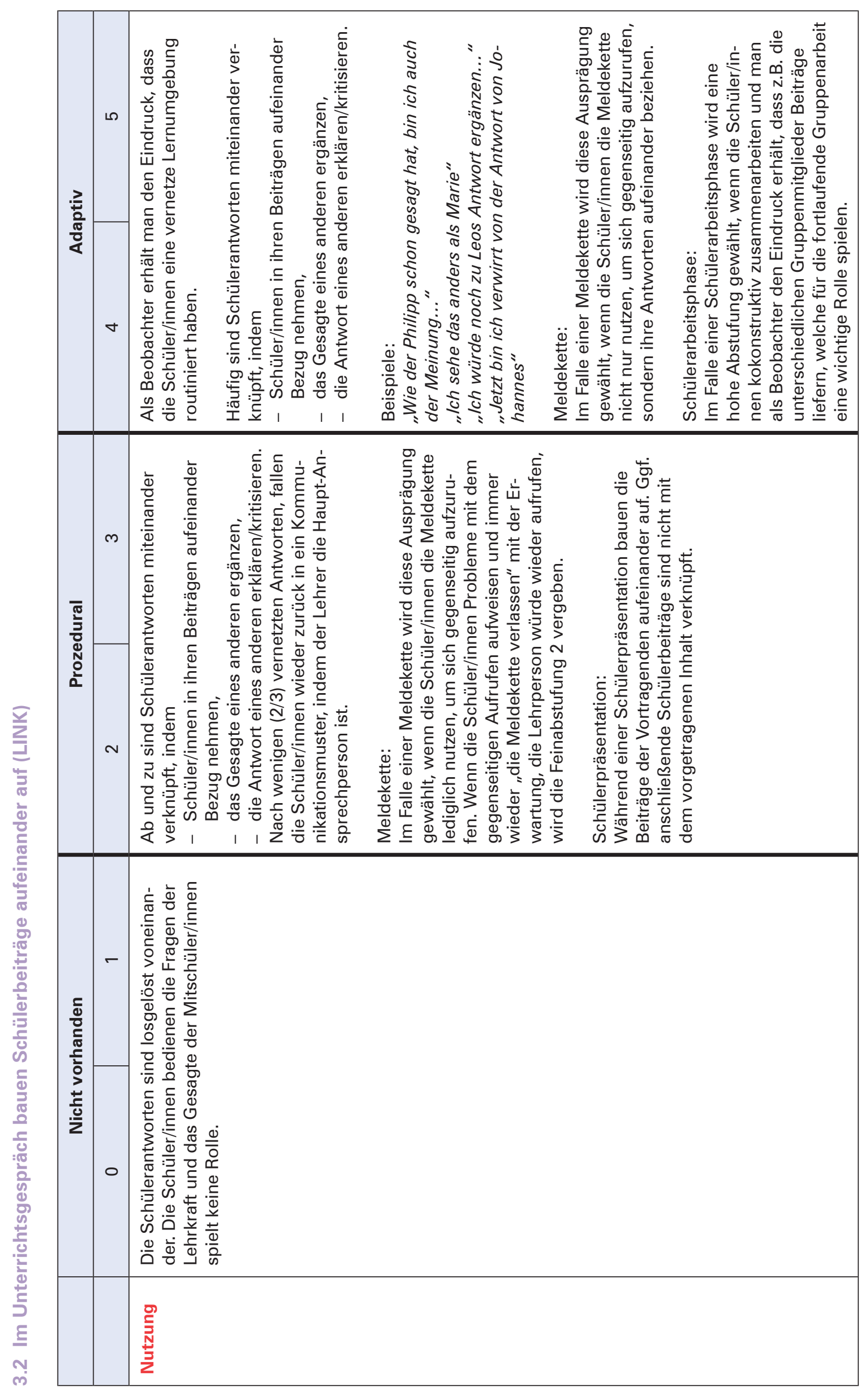




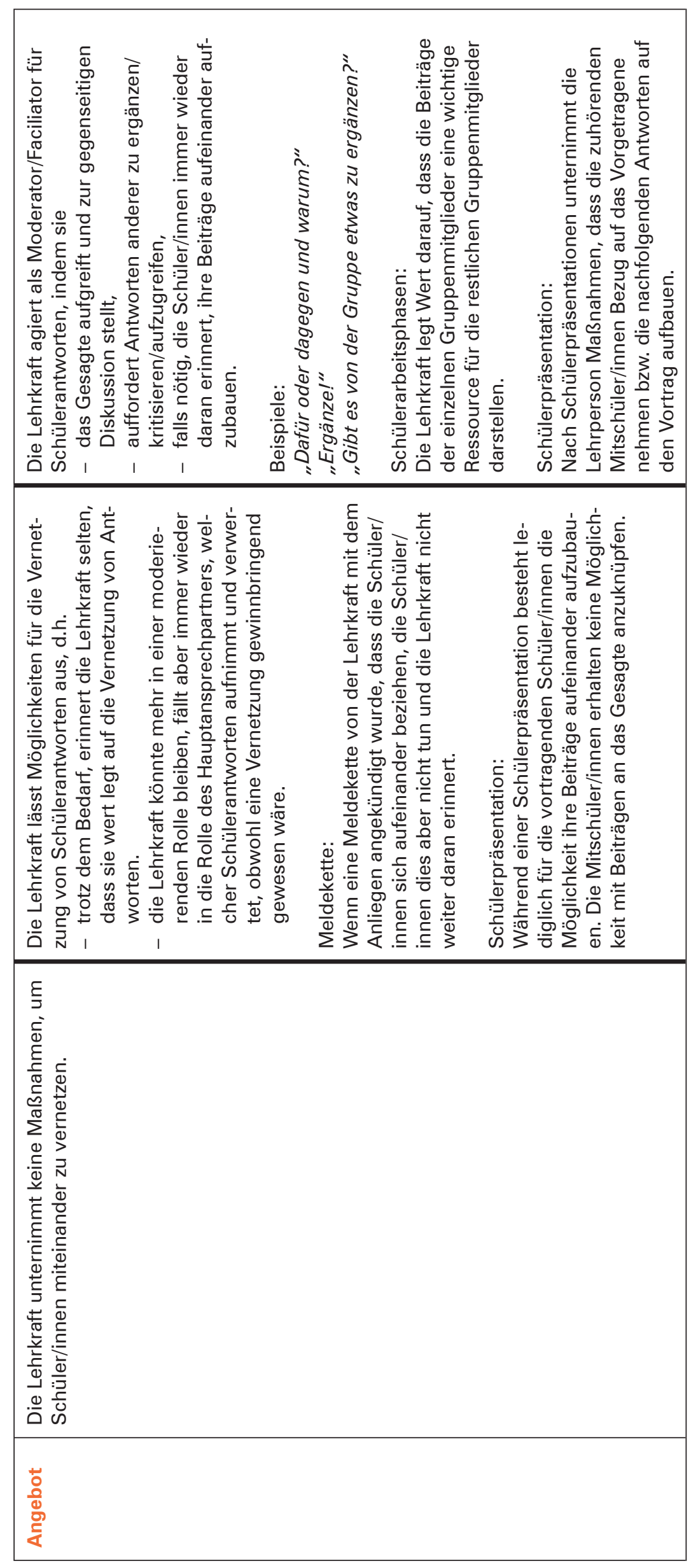




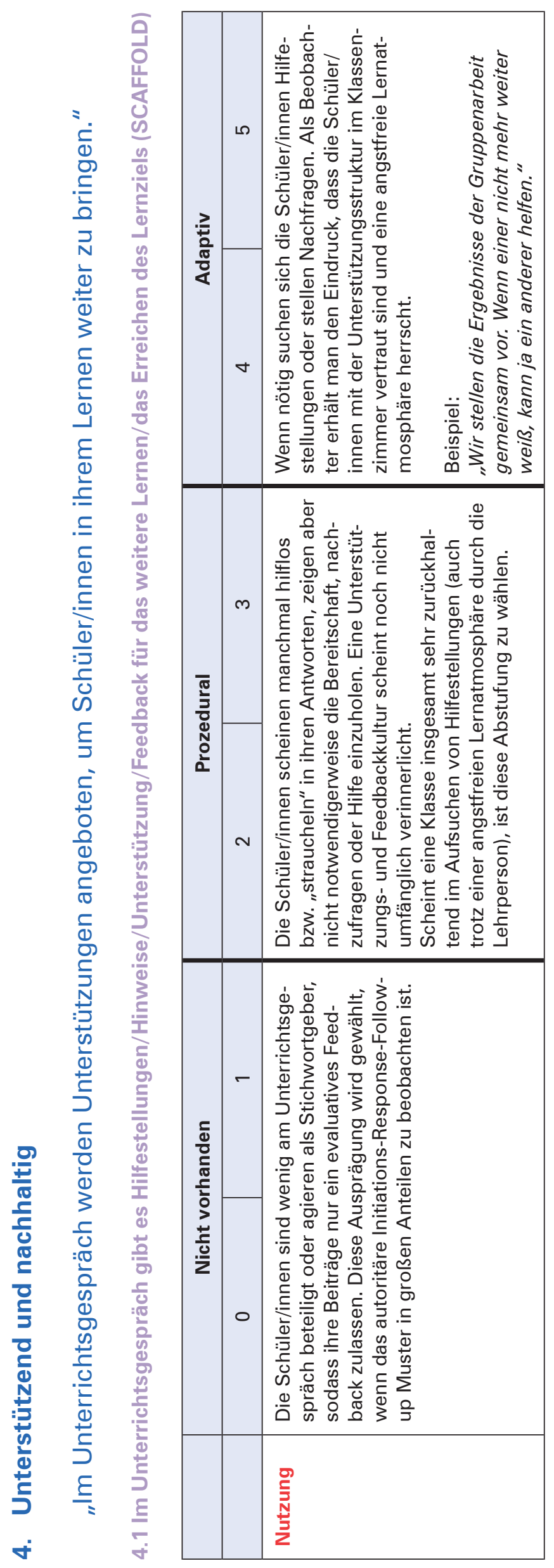




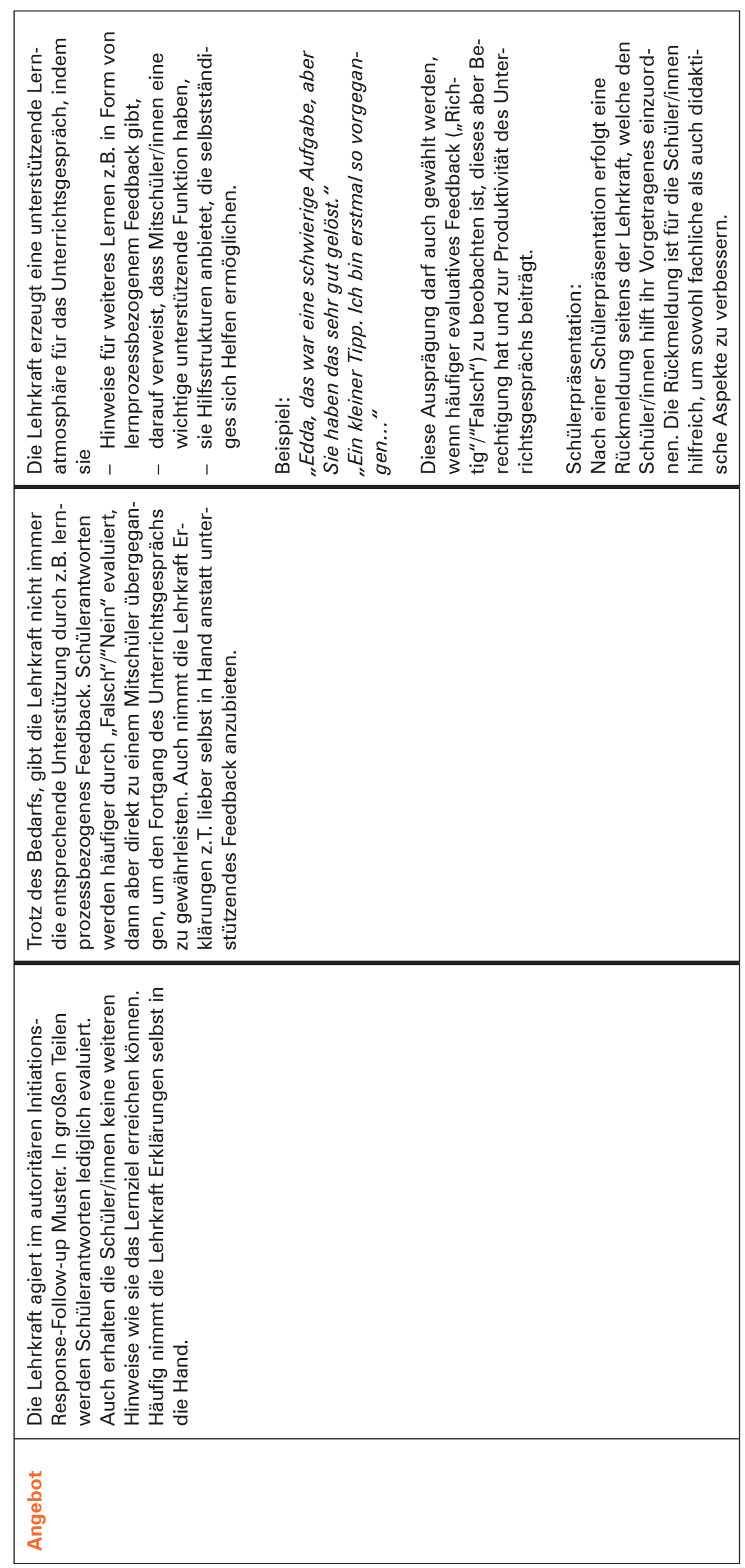




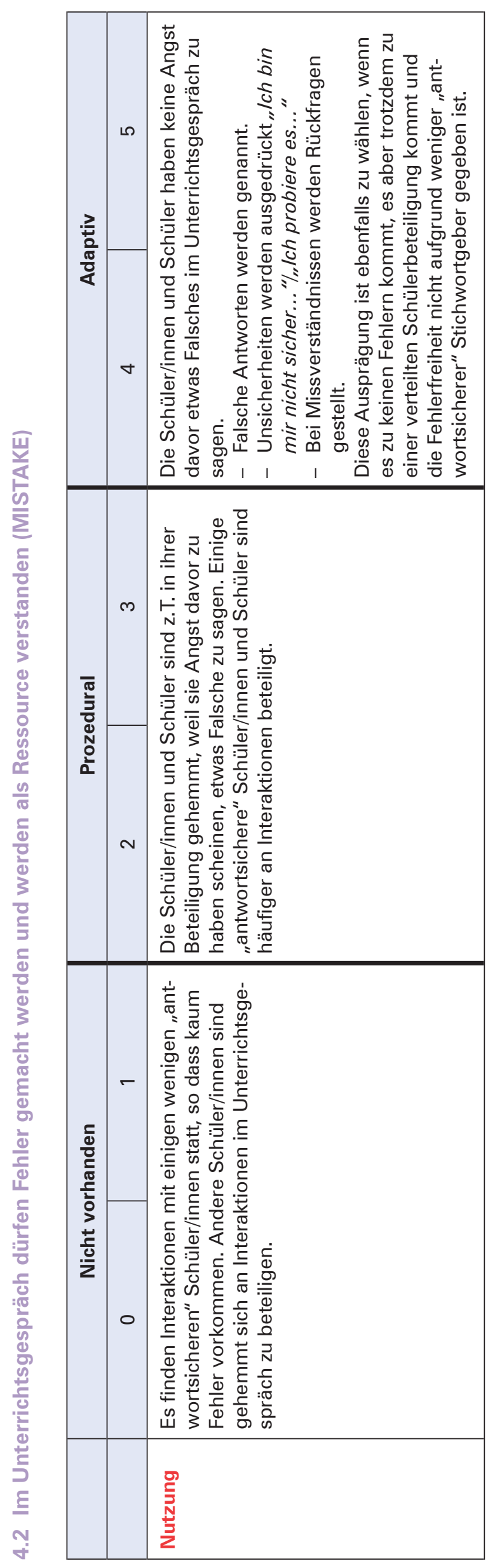




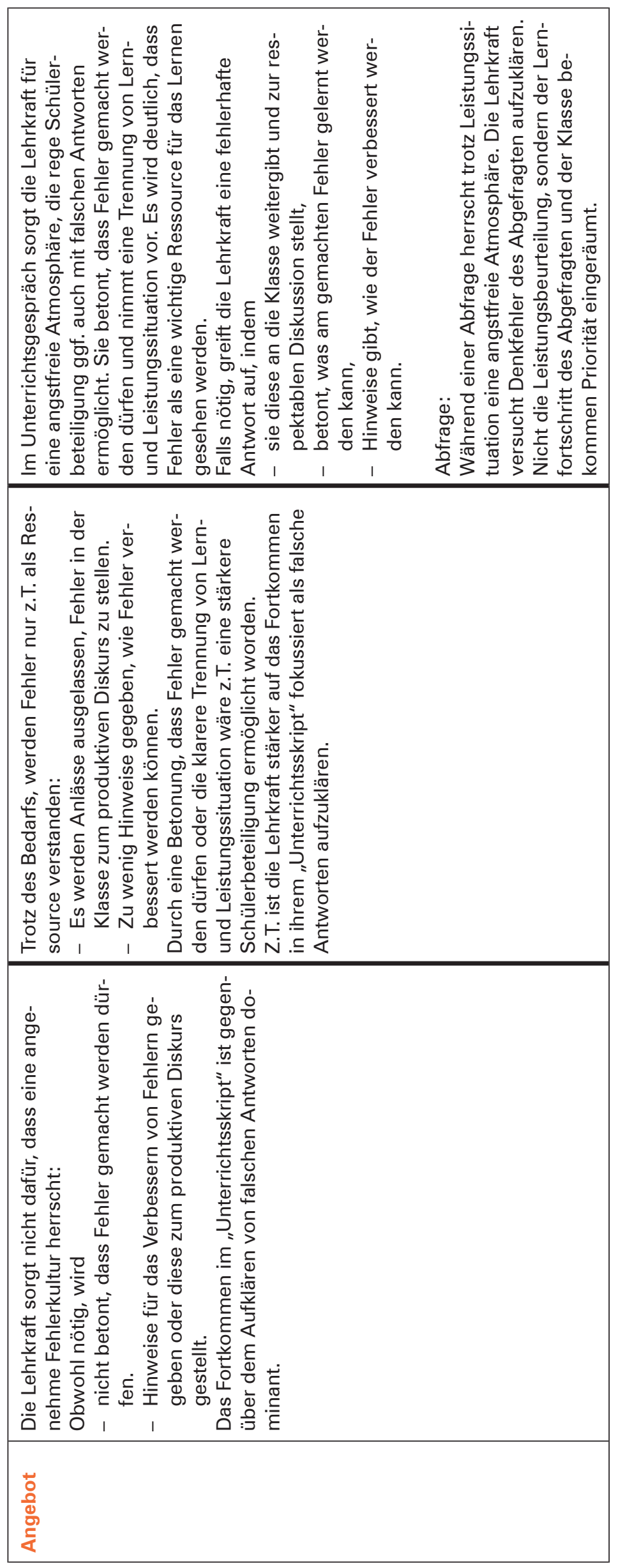




\section{Instrumentengüte}

Um die Reliabilität des Beobachtungsinstruments zu überprüfen, wurde die Einschätzung des gesamten Videomanuals durch zwei unabhängige, an Übungsmaterial trainierten Ratern vorgenommenen. Eine der beiden Personen stellte aufgrund der hohen Expertise im Feld der dialogischen Unterrichtsgesprächsführung den Masterrater dar. Alles Videomaterial wurde vom Masterrater blind für Messzeitpunkt und Fortbildungsangebot eingeschätzt. Um Reihenfolgeeffekte (Igler, Ohle-Peters, \& McElvany, 2018) gering zu halten, bewertete der Masterrater die Unterrichtsvideographien in entgegengesetzter Reihenfolge zum Zweitrater.

Im Folgenden werden verschiedene Reliabilitätsindices (in Anlehnung an Reznitskaya et al., 2016) für die vier Qualitätsdimensionen des Instruments, separat für die akkumulierten Lehreritems und Schüleritems abgebildet.

Tabelle 3.2: Übersicht über die Reliabilitäten und studienspezifische Anwendung des Videoinstruments Unterricht

\begin{tabular}{|c|c|c|c|c|c|c|}
\hline \multirow[t]{2}{*}{$\begin{array}{l}\text { Qualitäts- } \\
\text { dimension }\end{array}$} & \multirow[t]{2}{*}{$\begin{array}{c}\text { Aspekte eines produk- } \\
\text { tiven Unterrichtsge- } \\
\text { sprächs }\end{array}$} & \multicolumn{5}{|c|}{$\begin{array}{l}\text { Index für Reliabilität, studienspezifische } \\
\text { Anwendung des Videoinstruments }{ }^{1}\end{array}$} \\
\hline & & $\mathrm{ICC}^{2}$ & $a$ & Pearson $r$ & Spearman $\rho$ & $\begin{array}{c}\text { \% der Seg- } \\
\text { mente mit } \\
\text { max. 1-Punkt } \\
\text { Differenz }\end{array}$ \\
\hline \multirow[t]{2}{*}{$\begin{array}{l}\text { \#1 zielgerichtet } \\
\text { und strukturiert }\end{array}$} & $\begin{array}{l}\text { Score Angebot } \\
\text { (akkumuliert }^{3} \text { \#1.1- } \\
\text { \#1.3 Angebot Items) }\end{array}$ & .79 & .90 & .81 & .80 & 89.3 \\
\hline & $\begin{array}{l}\text { Score Nutzung } \\
\text { (akkumuliert }^{3} \text { \#1.1- } \\
\text { \#1.3 Nutzung Items) }\end{array}$ & .83 & .91 & .84 & .82 & 92.3 \\
\hline \multirow[t]{2}{*}{$\begin{array}{l}\text { \#2 aktivierend } \\
\text { und offen }\end{array}$} & $\begin{array}{l}\text { Score Angebot } \\
\text { (akkumuliert }^{3} \text { \#2.1- } \\
\text { \#2.3 Angebot Items) }\end{array}$ & .65 & .79 & .66 & .62 & 86.0 \\
\hline & $\begin{array}{l}\text { Score Nutzung } \\
\text { (akkumuliert }^{3} \text { \#2.1- } \\
\text { \#2.3 Nutzung Items) }\end{array}$ & .74 & .85 & .75 & .71 & 92.1 \\
\hline \multirow[t]{2}{*}{$\begin{array}{l}\text { \#3 interaktiv und } \\
\text { kumulativ }\end{array}$} & $\begin{array}{l}\text { Score Angebot } \\
\text { (akkumuliert }^{3} \text { \#3.1- } \\
\text { \#3.2 Angebot Items) }\end{array}$ & .64 & .78 & .64 & .65 & 80.9 \\
\hline & $\begin{array}{l}\text { Score Nutzung } \\
\text { (akkumuliert }^{3} \text { \#3.1- } \\
\text { \#3.2 Nutzung Items) }\end{array}$ & .70 & .82 & .70 & .70 & 89.5 \\
\hline \multirow[t]{2}{*}{$\begin{array}{l}\text { \#4 unterstützend } \\
\text { und nachhaltig }\end{array}$} & $\begin{array}{l}\text { Score Angebot } \\
\text { (akkumuliert }^{3} \text { \#4.1- } \\
\text { \#4.2 Angebot Items) }\end{array}$ & .65 & .82 & .72 & .68 & 90.5 \\
\hline & $\begin{array}{l}\text { Score Nutzung } \\
\text { (akkumuliert }^{3} \text { \#4.1- } \\
\text { \#4.2 Nutzung Items) }\end{array}$ & .65 & .81 & .69 & .66 & 90.5 \\
\hline
\end{tabular}

\footnotetext{
${ }^{1}$ In Anlehnung an Reznitskaya et al. (2016) wurden ICC, a, Pearson-Korrelation (angenommene Intervallskala), Spearman (angenommen als Ordinalskala) sowie \% -Segmente mit einem Maximum von 1 Punkt Abweichung als Maß für die Reliabilität berechnet. Im Allgemeinen wird für die Bewertungsskalen ein Intervall-Skalenniveau angenommen. Spearman wurde jedoch zusätzlich nach den Empfehlungen von Wirtz \& Caspar (2002) berechnet, um gegebenenfalls ordinalen Tendenzen der Ratingskalen gerecht zu werden.

${ }^{2}$ Wahl der two way-Methode (aufgrund eines Masterrater-Ratings in allen Fällen) zufällig (da sowohl die zu Bewertenden als auch die Rater aus einer größeren Population stammen), absolute Übereinstimmung (Ergebnisse der beiden Rater müssen gleich sein), einzeln (aufgrund der Tatsache, dass für die spätere Analyse die Werte verwendet wurden, die mit einem Masterrater erzielt wurden), ICC-Berechnung.

${ }^{3}$ Berechnungen basieren auf 1520 Fällen, da jedes Segment als einzelner Fall für jedes Item gelistet wurde.
} 
Alexander Gröschner, Dennis Hauk, Martina Alles, Richard Klöden, Ann-Kathrin Schindler, Maralena Weil \& Tina Seidel

\section{Gesprächs- und Lernkultur im evidenzbasierten Fortbildungsprogramm - Auswertung der videographierten Veranstaltungen der Fortbildungen}

\subsection{Theoretischer Hintergrund}

Die videobasierte Analyse der Fortbildungsworkshops zielt darauf, die Implementation und Entwicklung von effektiven Fortbildungselementen systematisch $\mathrm{zu}$ untersuchen. In Anlehnung an bestehende Forschung (Desimone, 2009; Sprott, 2019; Wilson, 2013) zeichnen sich effektive Fortbildungen - wie bereits in der Einleitung angedeutet - vor allem durch folgende Qualitätsmerkmale aus:

- Inhaltlicher Fokus: Der Fortbildungsfokus liegt auf einem spezifischen fachlichen, fachdidaktischen oder generell pädagogischen Inhalt, der eng mit der Unterrichtspraxis von Lehrpersonen verknüpft ist.

- Aktives Lernen: Lehrpersonen erhalten Möglichkeiten sich aktiv in die Fortbildung mit einzubringen, z.B. durch die gemeinsame Videoreflexion von eigenem Unterricht.

- Kohärenz: Das Fortbildungsangebot knüpft an den Bedarf und das Vorwissen der Lehrpersonen an und berücksichtigt schul- und systemspezifische Gegebenheiten.

- Zeitliche Dauer: Das Fortbildungsangebot sollte langfristig, z.B. über ein Schuljahr, ausgerichtet sein.

- Kollektive Partizipation: Lehrpersonen werden zu Diskussionen angeregt und erhalten die Möglichkeit, sich untereinander über Fortbildungsinhalte auszutauschen.

Der Faktor „Zeitliche Dauer“ wird durch das Messen von Länge und Dauer des jeweiligen Fortbildungsangebotes erfasst. Hingegen bilden die übrigen Merkmale, als „Tiefenstrukturen“ (Kunter \& Trautwein, 2013), die nicht direkt zu beobachtende Qualität der Interaktion (z.B. zwischen Fortbildner und Lehrpersonen) ab. Die Erfassung dieser „unsichtbaren“ Qualitätsmerkmale setzt einen interpretativen Zugang mittels kodierender Beobachtung und Schätzverfahren durch geschulte Expertinnen und Experten voraus (Pauli, 2014; Rakoczy \& Pauli, 2006).

\subsection{Datenerhebung und Videoaufzeichnung}

In der Studie „Dialogue II“ erfolgt die Einschätzung der Qualitätsmerkmale videobasiert auf Grundlage der aufgezeichneten Workshops der beiden Fortbildungsangebote. Mit Ausnahme des Auftaktworkshops, welcher in erster Linie zur Organisation der weiteren Abläufe diente, wurden alle Workshops der beiden Fortbildungsangebote für die Datenanalyse herangezogen. Insgesamt wurden knapp 27 Stunden Videomaterial aufgezeichnet und anschließend für die Datenanalyse in die Software Interact (Mangold, 2017) übertragen. 


\subsection{Kodierungsverfahren und Skalen}

\section{Schritt 1: Identifikation der Analyseeinheiten}

Um Aussagen über die Qualität der aufgezeichneten Fortbildungen treffen zu können, wird diese im ersten Analyseschritt in vergleichbare Analyseeinheiten unterteilt. Hierfür werden in der Videoforschung verschiedene Verfahren eingesetzt. Je nach gewähltem Vorgehen wird die Analyseeinheit abhängig von der Länge und Dauer konkreter Ereignisse, das „Event-Sampling“ (Bakeman \& Gottman, 1997), oder entsprechend vorher festgelegter Zeitintervalle, das „Time-Sampling“ (Hugener, 2006), festgelegt.

Das hier vorgestellte Beobachtungsinstrument orientiert sich am ereignisbasierten Verfahren, indem übergeordnete didaktisch-methodische Abschnitte innerhalb der Workshops (genannt „Facilitation Moves“), die in beiden Fortbildungsprogrammen gleichermaßen auftreten, identifiziert wurden. Die Einheiten wurden sowohl im Hinblick auf inhaltliche Indikatoren als auch auf den Start- und Endpunkt genau definiert (Tabelle 4.1). Innerhalb des Dialogue-II-Projekts wurde die Identifikation und Festlegung der Analyseeinheiten von den Facilitatoren im Nachgang der Durchführung der Fortbildungen vorgenommen.

Tabelle 4.1: Beschreibung der Analyseeinheiten des Videoinstruments der Fortbildungen

\begin{tabular}{|c|c|c|c|}
\hline Abschnitt & Beschreibung & Indikatoren & Start- und Endzeitpunkt \\
\hline $\begin{array}{l}\text { Abschnitt } 0 \\
\text { Organisation }\end{array}$ & $\begin{array}{l}\text { Es werden allge- } \\
\text { meine oder organi- } \\
\text { satorische Belange } \\
\text { thematisiert, die } \\
\text { nichts mit dem } \\
\text { inhaltlichen Fokus } \\
\text { des Workshops zu } \\
\text { tun haben. }\end{array}$ & $\begin{array}{l}\text { - Es findet eine Begrüßung statt. } \\
\text { - Der organisatorische Ablauf des Work- } \\
\text { shops wird vorgestellt. (Wichtig: So- } \\
\text { bald konkrete, inhaltliche, zu erreichen- } \\
\text { de Ziele thematisiert werden und auch } \\
\text { schon auf Inhalte eingegangen wird, } \\
\text { wird Abschnitt } 1 \text { kodiert.) } \\
\text { - Es findet eine Vorstellungsrunde statt. } \\
\text { - Es werden Erwartungen/Motivation } \\
\text { - } \text { abgefragt. } \\
\text { - Es findet eine Pause statt. } \\
\text { - Es findet eine Fragebogenhebung statt. } \\
\text { bart. } \\
\text { - Es geht um allgemeine Inhalte, die } \\
\text { nichts mit dem inhaltlichen Fokus des } \\
\text { Workshops zu tun haben, z.B. um Merk- } \\
\text { male effektiver Fortbildung. }\end{array}$ & $\begin{array}{l}\text { Anfang: Die Fortbildnerin/ } \\
\text { der Fortbildner beginnt } \\
\text { den Workshop/ unter- } \\
\text { bricht/endet den inhaltli- } \\
\text { chen Teil des Workshops. } \\
\text { Ende: Der inhaltliche Teil } \\
\text { des Workshops startet/ } \\
\text { wird fortgesetzt/endet. }\end{array}$ \\
\hline $\begin{array}{l}\text { Abschnitt } 1 \\
\text { Grundlagen- } \\
\text { wissen }\end{array}$ & $\begin{array}{l}\text { Es wird ein/e Ins- } \\
\text { truktion/Präsenta- } \\
\text { tion/Zusammen- } \\
\text { fassung/Dialog } \\
\text { von bisher nicht } \\
\text { thematisierten Fort- } \\
\text { bildungsinhalte } \\
\text { gegeben. }\end{array}$ & 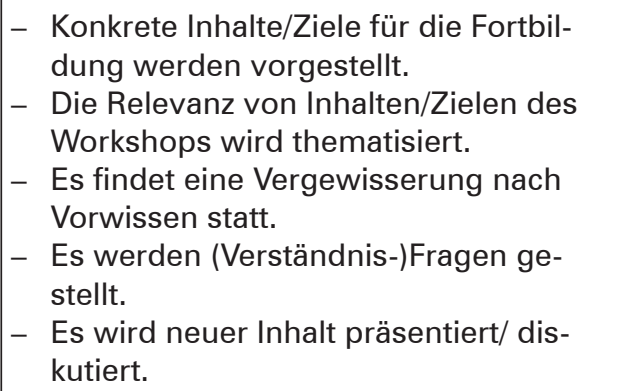 & $\begin{array}{l}\text { Anfang: Die Fortbildnerin/ } \\
\text { der Fortbildner leitet zu } \\
\text { einem neuen Inhalt über. } \\
\text { Ende: Die Präsentation } \\
\text { endet, es werden keine } \\
\text { Fragen/Diskussion mehr } \\
\text { zum neuen Inhalt gestellt } \\
\text { und es geht zu einer neu- } \\
\text { en Aktivität über. }\end{array}$ \\
\hline
\end{tabular}




\begin{tabular}{|c|c|c|c|}
\hline $\begin{array}{l}\text { Abschnitt 1a: } \\
\text { Wiederholung } \\
\text { des Wissens }\end{array}$ & $\begin{array}{l}\text { Wissen/Inhalte/Zie- } \\
\text { le aus vorherigen } \\
\text { Workshops werden } \\
\text { wiederholt. }\end{array}$ & $\begin{array}{l}\text { - Es wird ein Rückblick zum letzten Work- } \\
\text { shop gegeben. } \\
\text { - Es werden Ziele des letzten Workshops } \\
\text { thematisiert. } \\
\text { - Es werden bereits bekannte Inhalte zu- } \\
\text { sammengefasst. } \\
\text { - Es wird an bereits behandelte Inhalte } \\
\text { erinnert. }\end{array}$ & $\begin{array}{l}\text { Anfang: Die Fortbildnerin/ } \\
\text { der Fortbildner/Lehrperso- } \\
\text { nen leitet/leiten zu einem } \\
\text { bereits bekannten Inhalt } \\
\text { über. } \\
\text { Ende: Die Präsentation } \\
\text { endet, es werden keine } \\
\text { Fragen/Diskussion mehr } \\
\text { zum bereits bekannten In- } \\
\text { halt gestellt und es geht zu } \\
\text { einer neuen Aktivität über. }\end{array}$ \\
\hline $\begin{array}{l}\text { Abschnitt 2: } \\
\text { Gesprächs- } \\
\text { regeln }\end{array}$ & $\begin{array}{l}\text { Es werden Ge- } \\
\text { sprächs- und Feed- } \\
\text { backregeln thema- } \\
\text { tisiert. }\end{array}$ & $\begin{array}{l}\text { - Es werden Gesprächs- und Feedback- } \\
\text { regeln gesammelt/vorgestellt/aufge- } \\
\text { schrieben/diskutiert. } \\
\text { - } \text { Gesprächs- und Feedbackregeln umfas- } \\
\text { sen Regeln, die darauf zielen, wie sich } \\
\text { die Teilnehmenden gegenseitig unter- } \\
\text { stützen und sich gegenseitig Feedback } \\
\text { zu ihren Unterrichtsvideos geben. }\end{array}$ & $\begin{array}{l}\text { Anfang: Die Fortbildnerin/ } \\
\text { der Fortbildner/die Lehr- } \\
\text { personen thematisieren } \\
\text { Gesprächs- und Feedback- } \\
\text { regeln. } \\
\text { Ende: Es wird zu einem } \\
\text { anderen Inhalt übergegan- } \\
\text { gen. Gesprächs- und Feed- } \\
\text { backregeln werden nicht } \\
\text { weiter thematisiert. }\end{array}$ \\
\hline $\begin{array}{l}\text { Abschnitt 2a: } \\
\text { Wieder- } \\
\text { holung der } \\
\text { Gesprächs- } \\
\text { regeln }\end{array}$ & $\begin{array}{l}\text { Es werden Ge- } \\
\text { sprächs- und Feed- } \\
\text { backregeln wieder- } \\
\text { holt. }\end{array}$ & $\begin{array}{l}\text { - Es wird an Gesprächs- und Feedback- } \\
\text { regeln erinnert/hingewiesen. } \\
\text { - } \text { Gesprächs- und Feedbackregeln umfas- } \\
\text { sen Regeln, die darauf zielen, wie sich } \\
\text { die Teilnehmenden gegenseitig unter- } \\
\text { stützen und sich gegenseitig Feedback } \\
\text { zu ihren Unterrichtsvideos geben. }\end{array}$ & $\begin{array}{l}\text { Anfang: Die Fortbildnerin/ } \\
\text { der Fortbildner/die Lehr- } \\
\text { personen wiederholt } \\
\text { Gesprächs- und Feedback- } \\
\text { regeln. } \\
\text { Ende: Es wird zu einem } \\
\text { anderen Inhalt übergegan- } \\
\text { gen. Gesprächs- und Feed- } \\
\text { backregeln werden nicht } \\
\text { weiter thematisiert. }\end{array}$ \\
\hline $\begin{array}{l}\text { Abschnitt 3: } \\
\text { Bekanntgabe } \\
\text { Arbeitsauftrag }\end{array}$ & $\begin{array}{l}\text { Es wird ein Auftrag } \\
\text { zur Bearbeitung } \\
\text { gestellt. }\end{array}$ & $\begin{array}{l}\text { - } \text { Eine Präsentation der Aufgabenstellung } \\
\text { findet statt. } \\
\text { - } \text { Gruppen zur Bearbeitung werden ein- } \\
\text { geteilt. } \\
\text { - } \\
\text { Materialien zur Bearbeitung werden } \\
\text { ausgeteilt. } \\
\text { - } \\
\text { Fragen zum Auftrag werden gestellt } \\
\text { und geklärt. } \\
\text { - }\end{array}$ & $\begin{array}{l}\text { Anfang: Die Fortbildnerin/ } \\
\text { der Fortbildner stellt einen } \\
\text { Auftrag an die Lehrperso- } \\
\text { nen und erläutert die zu } \\
\text { bearbeitenden Aufgaben. } \\
\text { Ende: Der Auftrag ist allen } \\
\text { Teilnehmenden klar, es } \\
\text { gibt keine weiteren Fragen } \\
\text { und die Bearbeitung be- } \\
\text { ginnt. }\end{array}$ \\
\hline $\begin{array}{l}\text { Abschnitt 3a: } \\
\text { Bekanntgabe } \\
\text { eines reflexi- } \\
\text { ven Arbeits- } \\
\text { auftrages (nur } \\
\text { DVC) }\end{array}$ & $\begin{array}{l}\text { Es wird ein Auf- } \\
\text { trag zur reflektiven } \\
\text { Bearbeitung der } \\
\text { Unterrichtsvideos } \\
\text { gestellt }\end{array}$ & $\begin{array}{l}\text { - } \text { Es werden Videobeobachtungs- } \\
\text { aufträge gestellt. } \\
\text { - } \text { Es werden Leitfragen zur Video- } \\
\text { beobachtung präsentiert. } \\
\text { - } \text { Kontextinformationen zum Video } \\
\text { werden gegeben. } \\
\text { - Es wird das Vorgehen der Video- } \\
\text { beobachtung dargelegt und der } \\
\text { konkrete Ablauf/Aufteilung der } \\
\text { Videos in die einzelnen Workshops } \\
\text { thematisiert. } \\
\text { - Die Videos werden angesehen. }\end{array}$ & $\begin{array}{l}\text { Anfang: Die Fortbildnerin/ } \\
\text { der Fortbildner leitet die } \\
\text { Videobeobachtung ein. Er/ } \\
\text { Sie stellt den konkreten } \\
\text { Ablauf dar und nennt Kon- } \\
\text { textinformation und Leit- } \\
\text { fragen zur Beobachtung. } \\
\text { Ende: Der Auftrag zur } \\
\text { Videobeobachtung sowie } \\
\text { die Leitfragen und der } \\
\text { Kontext des Videos ist } \\
\text { allen Teilnehmenden klar. } \\
\text { Die eigentliche Videobe- } \\
\text { obachtung beginnt. }\end{array}$ \\
\hline
\end{tabular}




\begin{tabular}{|c|c|c|c|}
\hline $\begin{array}{l}\text { Abschnitt 4: } \\
\text { Arbeitsphase }\end{array}$ & $\begin{array}{l}\text { Es findet eine Aus- } \\
\text { einandersetzung } \\
\text { mit Fortbildungsin- } \\
\text { halten (Einzelarbeit, } \\
\text { Klein-, Großgrup- } \\
\text { pen oder Plenum) } \\
\text { statt. }\end{array}$ & 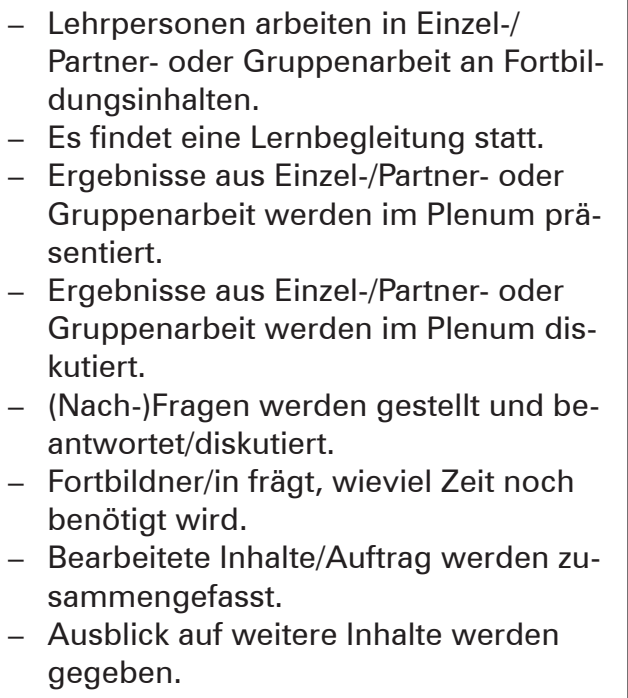 & $\begin{array}{l}\text { Anfang: Lehrpersonen } \\
\text { beginnen mit der Einzel-/ } \\
\text { Partner- oder Gruppen- } \\
\text { arbeit. } \\
\text { Ende: Die Bearbeitung/ } \\
\text { Nachbesprechung der Ak- } \\
\text { tivität endet und es geht zu } \\
\text { einer neuen Aktivität über. } \\
\text { Kurz vor Satzbeginn wird } \\
\text { das Ende des Abschnitts } \\
\text { gesetzt. }\end{array}$ \\
\hline $\begin{array}{l}\text { Abschnitt 4a: } \\
\text { Reflexive } \\
\text { Arbeitsphase } \\
\text { (nur DVC) }\end{array}$ & $\begin{array}{l}\text { Es findet eine Aus- } \\
\text { einandersetzung } \\
\text { mit Fortbildungsin- } \\
\text { halten (Einzelarbeit, } \\
\text { Klein-, Großgrup- } \\
\text { pen oder Plenum) } \\
\text { auf Basis eigener } \\
\text { Unterrichts-videos } \\
\text { statt. }\end{array}$ & $\begin{array}{l}\text { - Es wird beschrieben und erläutert, was } \\
\text { Lehrpersonen im Video beobachtet } \\
\text { haben. } \\
\text { - Ereignisse im Video werden diskutiert. } \\
\text { - Es findet eine Lernbegleitung statt. } \\
\text { - Es findet ein Austausch über die eigene } \\
\text { Unterrichtspraxis statt. }\end{array}$ & $\begin{array}{l}\text { Anfang: Lehrpersonen } \\
\text { beginnen mit der Einzel-/ } \\
\text { Partner- oder Gruppen- } \\
\text { arbeit. } \\
\text { Ende: Die Bearbeitung/ } \\
\text { Nachbesprechung der Ak- } \\
\text { tivität endet und es geht zu } \\
\text { einer neuen Aktivität über. } \\
\text { Kurz vor Satzbeginn wird } \\
\text { das Ende des Abschnitts } \\
\text { gesetzt. }\end{array}$ \\
\hline $\begin{array}{l}\text { Abschnitt 5: } \\
\text { Zusammen- } \\
\text { fassung }\end{array}$ & $\begin{array}{l}\text { Es wird eine Zu- } \\
\text { sammenfassung } \\
\text { des Workshops } \\
\text { gegeben. }\end{array}$ & $\begin{array}{l}\text { - } \text { Allgemeine Fragen zu Inhalten/ Aufträ- } \\
\text { gen werden gestellt. } \\
\text { - Es findet eine inhaltliche Zusammen- } \\
\text { fassung statt. } \\
\text { - } \\
\text { Abschließende Inhalte werden zur Ab- } \\
\text { rundung angebracht. }\end{array}$ & $\begin{array}{l}\text { Anfang: Die Fortbildnerin/ } \\
\text { der Fortbildner fasst die } \\
\text { Inhalte zusammen und es } \\
\text { werden noch letzte Fragen } \\
\text { gestellt. } \\
\text { Ende: Es geht zu Organi- } \\
\text { satorischem über bzw. der } \\
\text { Workshop endet. }\end{array}$ \\
\hline
\end{tabular}

\section{Schritt 2: Kodierung der Qualitätsdimensionen}

Jeder Fortbildungsabschnitt wurde im zweiten Schritt hinsichtlich der Qualitätsmerkmale von zwei Kodiererinnen und Kodierern unabhängig eingeschätzt. Die dafür benötigten Beobachtungsschwerpunkte („Items“) sind hoch inferent, d.h. sie besitzen einen großen Anteil interpretativer Schlussfolgerungen, um die Komplexität des jeweiligen Qualitätsmerkmals erfassen zu können (Clausen, Reusser, \& Klieme, 2003). Beide Kodiererinnen und Kodierer kodierten das vollständige Videomaterial der Fortbildungen. Die Einschätzung jedes Beobachtungsschwerpunktes erfolgt dabei

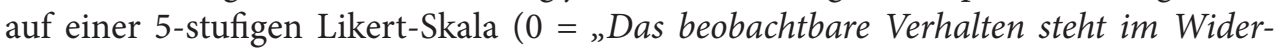
spruch zum Item." bis 4 = „Das Item ist optimal über einen längeren Zeitraum ausgeprägt.") und bezieht sowohl das Verhalten der Fortbildnerinnen und Fortbildner als auch das der Lehrkräfte mit ein. Da sich die didaktisch-methodischen Implikationen jedes Fortbildungsabschnittes unterschieden (z.B. ist in einer Organisationsphase kein inhaltlicher Diskurs zu erwarten), wurden vorab für die verschiedenen Fortbildungsabschnitte die zu erwartenden Items festgelegt bzw. nicht zu erwartende Items von der Einschätzung ausgeschlossen. Im Anschluss wurden Übereinstimmungskoeffizienten der beiden unabhängigen Kodiererinnen und Kodierer für jede Qualitätsdimension berechnet (4.2). Die Übereinstimmungen können hierbei als akzeptabel betrachtet werden. 
Tabelle 4.2: Übersicht über die Reliabilitäten des Videoinstruments der Fortbildungen

\begin{tabular}{lccccc}
\hline Qualitätsdimension & ICC $^{2}$ & $\alpha$ & $\begin{array}{c}\text { Pearson } \\
\boldsymbol{r}\end{array}$ & $\begin{array}{c}\text { Spear- } \\
\text { man } \rho\end{array}$ & $\begin{array}{c}\text { \% der Segmente } \\
\text { mit max. 1-Punkt } \\
\text { Differenz }\end{array}$ \\
\hline Lernatmosphäre & .75 & .74 & .65 & .65 & 88.48 \\
\hline $\begin{array}{l}\text { Inhaltlicher Fokus } \\
\text { Kohärentes und aktives }\end{array}$ & .70 & -1 & .55 & .58 & 89.56 \\
\hline Lernen & .76 & .86 & .64 & .66 & 88.95 \\
\hline Kollektive Partizipation & .76 & .68 & .68 & .68 & 85.88 \\
\hline
\end{tabular}

${ }^{1}$ Die Erfassung dieses Qualitätsmerkmals basiert auf zwei Einzelitems.

Die nachfolgenden Tabellen geben einen Überblick über die im Beobachtungsinstrument enthaltenen Items sowie die dazu gehörenden Indikatoren.

Zur Einschätzung der Qualitätsmerkmale wurde folgende Abstufung der Einschätzungen gewählt:

Einschätzung:

$0=\quad$ Das beobachtbare Verhalten steht im Widerspruch zum Item.

$1=\quad$ Das Item ist nicht beobachtbar.

$2=\quad$ Das Item ist schwach über einen sehr begrenzten Zeitraum ausgeprägt.

$3=\quad$ Das Item ist schwach über einen längeren Zeitraum ausgeprägt. ODER Das Item ist stark über einen kurzen Zeitraum ausgeprägt.

$4=\quad$ Das Item ist optimal über einen längeren Zeitraum ausgeprägt. 
Qualitätsmerkmal "Lernatmosphäre"

Quelle: Eigenentwicklung

Tabelle 4.3: Beobachtungsschwerpunkte für das Qualitätsmerkmal "Lernatmosphäre"

\begin{tabular}{|c|c|c|c|}
\hline Item & $\begin{array}{l}\text { Beschreibung } \\
\text { In dem einzuschätzen- } \\
\text { den Abschnitt... }\end{array}$ & Indikatoren & $\begin{array}{l}\text { Berücksichtigt im } \\
\text { Abschnitt }\end{array}$ \\
\hline 1.1_vertrauen & $\begin{array}{l}\text {... herrscht eine ange- } \\
\text { nehme Atmosphäre. }\end{array}$ & $\begin{array}{l}\text { - } \text { gegenseitiges Aussprechen lassen } \\
\text { - } \text { freies Äußern von Ideen \& Meinun- } \\
\text { gen } \\
\text { - } \\
\text { Blickkontakt und offene Körperhal- } \\
\text { tung } \\
\text { - }\end{array}$ & Alle \\
\hline 1.2_aufmerksamkeit & $\begin{array}{l}\text {... werden Redebei- } \\
\text { träge aufmerksam } \\
\text { verfolgt. }\end{array}$ & $\begin{array}{ll}\text { - } & \text { aktives Zuhören } \\
\text { - } & \text { Gehör für Beiträge/die Beiträge von } \\
& \text { sprechenden Personen } \\
\text { - } & \text { Herausstellen der Bedeutung von } \\
& \text { Redebeiträgen } \\
\text { - } & \text { Blickkontakt zu sprechenden Perso- } \\
& \text { nen } \\
- & \text { ungeteilter Fokus (keine Ablenkung } \\
& \text { durch z.B. private Handynutzung) }\end{array}$ & Alle \\
\hline 1.3_fragen & $\begin{array}{l}\text {... wird offen mit Fra- } \\
\text { gen umgegangen. }\end{array}$ & $\begin{array}{l}\text { - Raum für (Nach-)Fragen } \\
\text { - Stellen von (Nach-)Fragen } \\
\text { - Eingehen auf (Nach-)Fragen }\end{array}$ & Alle \\
\hline
\end{tabular}

Qualitätsmerkmal „Inhaltlicher Fokus“

Quelle: Eigenentwicklung, in Anlehnung an Desimone (2009)

Tabelle 4.4: Beobachtungsschwerpunkte für das Qualitätsmerkmal "Inhaltlicher Fokus"

\begin{tabular}{|c|c|c|c|}
\hline Item & $\begin{array}{l}\text { Beschreibung } \\
\text { In dem einzuschätzen- } \\
\text { den Abschnitt... }\end{array}$ & Indikatoren & $\begin{array}{l}\text { Berücksichtigt im } \\
\text { Abschnitt }\end{array}$ \\
\hline 2.1_wissen & $\begin{array}{l}\text { - ... wird pädago- } \\
\text { gisches Wissen in } \\
\text { Bezug auf die Fort- } \\
\text { bildungsinhalte } \\
\text { eingebracht. }\end{array}$ & $\begin{array}{l}\text { - Es erfolgt ein Input pädagogischer } \\
\text { Fachinhalte (durch Vortrag, Texte, } \\
\text { Arbeitsblätter, Präsentationsfolien, } \\
\text { etc.). } \\
\text { - Pädagogische Fachinhalte werden } \\
\text { im Rahmen einer Diskussion auf- } \\
\text { gegriffen. }\end{array}$ & $1 / 1 a, 4 / 4 a$ \\
\hline 2.2_curriculum & $\begin{array}{l}\text { - } \ldots \text { wird das in der } \\
\text { Fortbildung erwor- } \\
\text { bene Wissen mit } \\
\text { konkreten Unter- } \\
\text { richtsthemen/ Fach- } \\
\text { inhalten verknüpft. }\end{array}$ & $\begin{array}{l}\text { - Es wird über den Lehrplan bzw. } \\
\text { über die im Lehrplan enthaltenen } \\
\text { Themen gesprochen. }\end{array}$ & $1 / 1 a, 4 / 4 a$ \\
\hline
\end{tabular}


Qualitätsmerkmal "Kohärentes und aktives Lernen“

Quelle: Eigenentwicklung, in Anlehnung an Desimone (2009)

Tabelle 4.5: Beobachtungsschwerpunkte für das Qualitätsmerkmal "Kohärentes und aktives Lernen"

\begin{tabular}{|c|c|c|c|}
\hline Item & $\begin{array}{l}\text { Beschreibung } \\
\text { In dem einzuschätzen- } \\
\text { den Abschnitt... }\end{array}$ & Indikatoren & $\begin{array}{l}\text { Berücksichtigt im } \\
\text { Abschnitt }\end{array}$ \\
\hline 3.1_beschreiben & $\begin{array}{l}\text {... werden in einem } \\
\text { unterrichtsbezogenen } \\
\text { Reflexionsgespräch } \\
\text { verschiedene Unter- } \\
\text { richtsaspekte beschrie- } \\
\text { ben. }\end{array}$ & $\begin{array}{l}\text { - Es findet ein wertneutraler Aus- } \\
\text { tausch über eine erfahrene/beob- } \\
\text { achtete Unterrichtssituation statt. }\end{array}$ & $4 / 4 a$ \\
\hline 3.2_bewerten & $\begin{array}{l}\text {... werden in einem } \\
\text { unterrichtsbezogenen } \\
\text { Reflexionsgespräch } \\
\text { verschiedene Unter- } \\
\text { richtsaspekte bewertet. }\end{array}$ & $\begin{array}{l}\text { - Der Austausch über eine erfahrene/ } \\
\text { beobachtete Unterrichtssituation ist } \\
\text { wertend. }\end{array}$ & $1 / 1 a, 4 / 4 a$ \\
\hline 3.3_erfahrung & $\begin{array}{l}\text {... werden eigene Lehr- } \\
\text { erfahrungen in Bezug } \\
\text { auf die Umsetzung } \\
\text { spezifischer Fortbil- } \\
\text { dungsinhalte themati- } \\
\text { siert. }\end{array}$ & 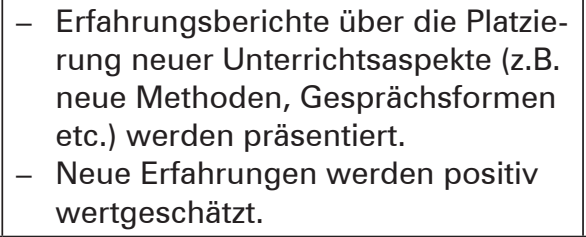 & $1 / 1 a, 3 / 3 a, 4 / 4 a$ \\
\hline 3.4_planung & $\begin{array}{l}\text {... wird über Unter- } \\
\text { richtsplanung gespro- } \\
\text { chen. }\end{array}$ & $\begin{array}{l}\text { - Es wird über alltägliches (konkre- } \\
\text { tes, etc.) Planungsverhalten ge- } \\
\text { sprochen } \\
\text { - Es wird über Ziele und Routinen } \\
\text { der Unterrichtsplanung gespro- } \\
\text { chen. }\end{array}$ & $0,3,4 / 4 a$ \\
\hline 3.5_material & $\begin{array}{l}\text {... wird eigener Unter- } \\
\text { richt auf Basis von Ma- } \\
\text { terialien thematisiert. }\end{array}$ & $\begin{array}{l}\text { - Unterricht wird auf Basis verschie- } \\
\text { dener Materialien (z.B. Literatur, } \\
\text { Fallvignetten, Planungsskizzen, } \\
\text { Videos). }\end{array}$ & $1 / 1 a, 4 / 4 a$ \\
\hline 3.6_transfer & $\begin{array}{l}\text {... werden Möglichkei- } \\
\text { ten des Transfers von } \\
\text { Fortbildungsinhalten in } \\
\text { den eigenen Unterricht } \\
\text { thematisiert. }\end{array}$ & $\begin{array}{l}\text { - Es werden Handlungsanleitungen } \\
\text { (Methoden/Strategien) für den } \\
\text { Unterricht besprochen. } \\
\text { - Es werden Handlungsalternativen } \\
\text { vorgeschlagen. }\end{array}$ & $1 / 1 a, 4 / 4 a$ \\
\hline 3.7_feedback & $\begin{array}{l}\text {... erhalten die Fort- } \\
\text { bildner oder die teil- } \\
\text { nehmenden Lehrperso- } \\
\text { nen ein Feedback. }\end{array}$ & \begin{tabular}{|l} 
- Lehrpersonen erhalten eine Rück- \\
meldung zu konkreten Lernfort- \\
schritten oder der Bearbeitung von \\
Arbeitsaufträgen. \\
- Die Leistung der/des Fortbildner(s) \\
wird von den teilnehmenden Lehr- \\
personen rückgemeldet. \\
\end{tabular} & $\begin{array}{l}\text { Alle außer } \\
\text { Abschnitt } 3\end{array}$ \\
\hline 3.8_diskussion & $\begin{array}{l}\text {... wird hinsichtlich } \\
\text { eines konkreten Pro- } \\
\text { blems über Lösungs- } \\
\text { möglichkeiten und } \\
\text {-ansätze gesprochen. }\end{array}$ & $\begin{array}{l}\text { - Es werden Handlungsalternativen } \\
\text { (Methoden und Strategien) aufge- } \\
\text { zeigt/diskutiert. }\end{array}$ & $4 / 4 a$ \\
\hline
\end{tabular}


Qualitätsmerkmal "Kollektive Partizipation"

Quelle: Eigenentwicklung, in Anlehnung an Desimone (2009)

Tabelle 4.6: Beobachtungsschwerpunkte für das Qualitätsmerkmal „Kollektive Partizipation“

\begin{tabular}{|c|c|c|c|}
\hline Item & $\begin{array}{l}\text { Beschreibung } \\
\text { In dem einzuschätzen- } \\
\text { den Abschnitt... }\end{array}$ & Indikatoren & $\begin{array}{l}\text { Berücksichtigt im } \\
\text { Abschnitt }\end{array}$ \\
\hline 4.1_adaptivität & $\begin{array}{l}\text {... werden die Gestal- } \\
\text { tungswünsche der teil- } \\
\text { nehmenden Lehrperso- } \\
\text { nen berücksichtigt. }\end{array}$ & $\begin{array}{l}\text { - Lehrpersonen können eigene Ideen } \\
\text { hinsichtlich der Konzeption und Ge- } \\
\text { staltung der Fortbildung einbringen. } \\
\text { - Lehrpersonen besitzen Wahlfrei- } \\
\text { heiten z.B. in Bezug auf inhaltliche } \\
\text { Vertiefungen, die Bearbeitungsrei- } \\
\text { henfolge von Aufgaben, Abläufen, } \\
\text { Umgang mit Materialien. }\end{array}$ & Alle Abschnitte \\
\hline 4.2_diskurs & $\begin{array}{l}\text {... wird auf Beiträge } \\
\text { von sprechenden Per- } \\
\text { sonen Bezug genom- } \\
\text { men. }\end{array}$ & 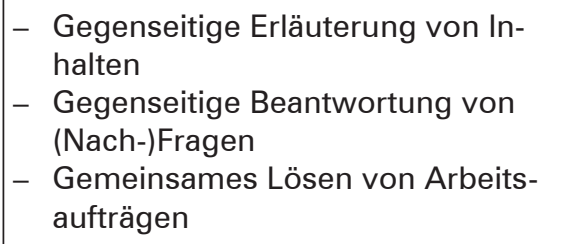 & $3 a, 4 / 4 a$ \\
\hline 4.3_moderation & $\begin{array}{l}\text {... findet ein (selbst)or- } \\
\text { ganisierter/moderierter } \\
\text { Gesprächsaustausch } \\
\text { der teilnehmenden } \\
\text { Lehrpersonen statt. }\end{array}$ & $\begin{array}{l}\text { - Fortbildner/Lehrpersonen leiten } \\
\text { und steuern das Gespräch (selbst- } \\
\text { ständig). } \\
\text { - Die Reihenfolge von Sprechern wird } \\
\text { durch den Fortbildner/Lehrer be- } \\
\text { stimmt. }\end{array}$ & $0,3,4 / 4 a$ \\
\hline
\end{tabular}




\section{Alexander Gröschner, Ann-Kathrin Schindler, Maralena Weil, Ricardo Böheim \& Tina Seidel}

\section{Instrumente zur Befragung von Lehrpersonen}

Wie in der Einleitung dieses Bandes beschrieben, war ein Ziel der „Dialogue II“Studie, die persönlichen Einstellungen und die Wahrnehmung der Fortbildung sowie Transfermöglichkeiten der Lehrpersonen zu berücksichtigen. In die Erhebungsinstrumente gingen dafür in der ersten Förderphase eingesetzte, etablierte Skalen sowie neue, z.T. adaptierte Instrumente ein.

Die teilnehmenden Lehrpersonen in beiden Fortbildungen (DVC und DIP) wurden zu fünf Messzeitpunkten schriftlich befragt. In der nachstehenden Abbildung 5.1 ist der Erhebungsablauf für beide Fortbildungsformate vergleichend dargestellt.

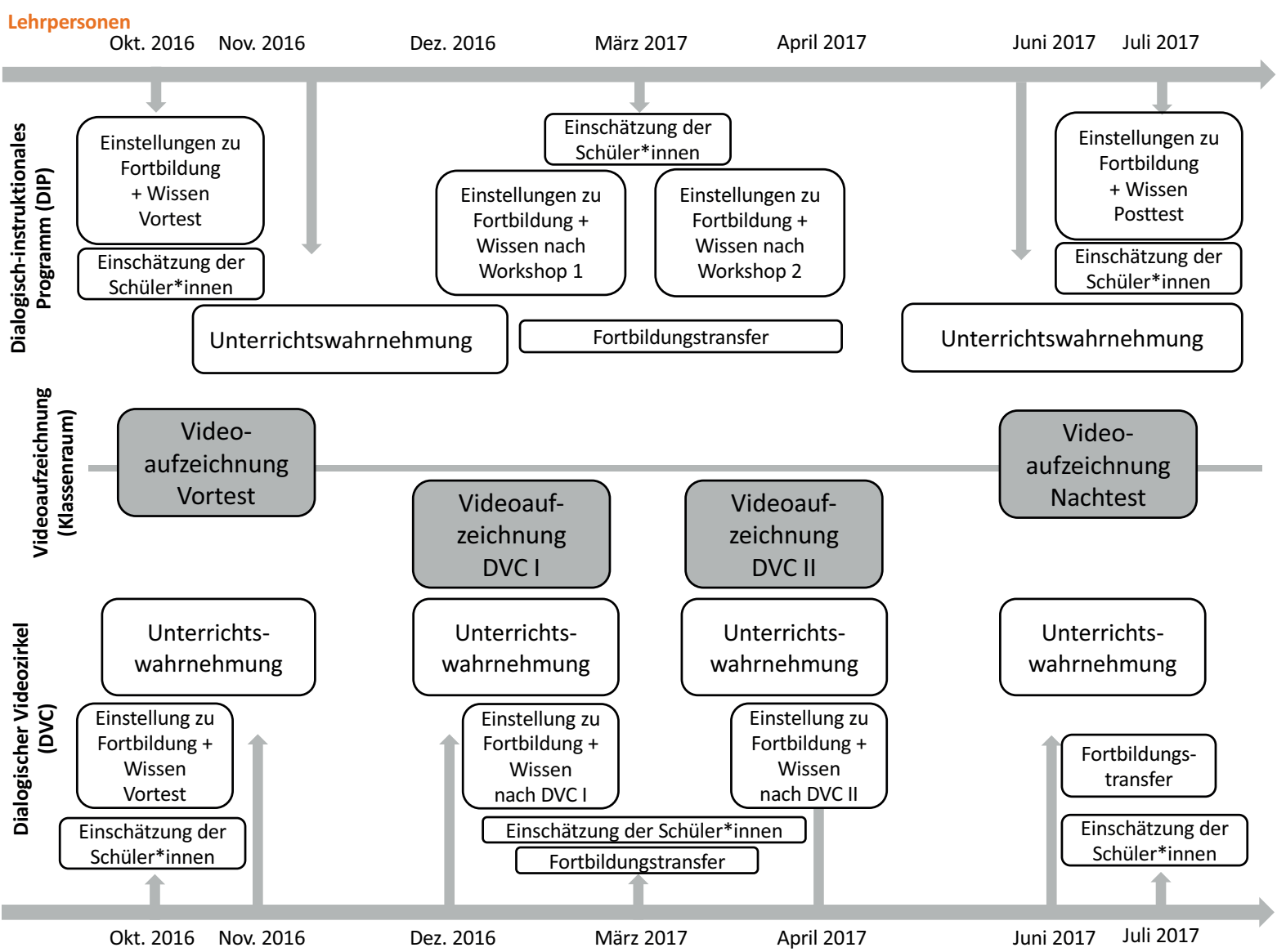

Abbildung 5.1: Übersicht der Erhebungszeitpunkte und Messinstrumente bei Lehrkräften beider Fortbildungsangebote 


\subsection{Einstellungen der Lehrpersonen}

Skala Lehrerselbstwirksamkeit SK_L_sw

Quelle: adaptiert von Schwarzer \& Schmitz, 1999

\section{Ausprägung: 3 stimmt genau \\ 2 stimmt eher \\ 1 stimmt kaum \\ 0 stimmt nicht}

Anweisung: Wie würden Sie sich selbst anhand der folgenden Aussagen einschätzen?

\begin{tabular}{|l|l|}
\hline VARIABLE & Item - Wortlaut \\
\hline Sw1 & $\begin{array}{l}\text { Ich bin mir sicher, dass ich auch mit den problematischen Schülern in } \\
\text { guten Kontakt kommen kann, wenn ich mich darum bemühe. }\end{array}$ \\
\hline Sw2 & $\begin{array}{l}\text { Ich weiß, dass ich zu den Eltern guten Kontakt halten kann, selbst in } \\
\text { schwierigen Situationen. }\end{array}$ \\
\hline Sw3 & $\begin{array}{l}\text { Ich weiß, dass ich es schaffe, selbst den problematischsten Schülern } \\
\text { den prüfungsrelevanten Stoff zu vermitteln. }\end{array}$ \\
\hline Sw4 & $\begin{array}{l}\text { Ich bin mir sicher, dass ich mich in Zukunft auf individuelle Probleme } \\
\text { der Schüler noch besser einstellen kann. }\end{array}$ \\
\hline Sw5 & $\begin{array}{l}\text { Selbst wenn mein Unterricht gestört wird, bin ich mir sicher, die not- } \\
\text { wendige Gelassenheit bewahren zu können. }\end{array}$ \\
\hline Sw6 & $\begin{array}{l}\text { Selbst wenn es mir mal nicht so gut geht, kann ich doch im Unterricht } \\
\text { immer noch gut auf die Schüler eingehen. }\end{array}$ \\
\hline Sw7 & $\begin{array}{l}\text { Auch wenn ich mich noch so sehr für die Entwicklung meiner Schüler } \\
\text { engagiere, weiß ich, dass ich nicht viel ausrichten kann. }\end{array}$ \\
\hline Sw8 & $\begin{array}{l}\text { Ich bin mir sicher, dass ich kreative Ideen entwickeln kann, mit denen } \\
\text { ich ungünstige Unterrichtsstrukturen verändere. }\end{array}$ \\
\hline Sw9 & \begin{tabular}{l} 
Ich traue mir zu, die Schüler für neue Projekte zu begeistern. \\
\hline Sw10
\end{tabular} \\
$\begin{array}{l}\text { Ich kann innovative Veränderungen auch gegenüber skeptischen Kol- } \\
\text { legen durchsetzen. }\end{array}$ \\
\hline
\end{tabular}


Skala Einstellungen zum konstruktiven Lernen und Lehren - konstruktiv SK_L_ einst_LL_Konstruktiv

Quelle: Kunter et al., 2017

$\begin{array}{ll}\text { Ausprägung: } & 3 \text { trifft zu } \\ & 2 \text { trifft eher } z u \\ & 1 \text { trifft eher nicht zu } \\ & 0 \text { trifft nicht } z u\end{array}$

Anweisung: Wie sehr stimmen Sie den folgenden Aussagen zu?

\begin{tabular}{|l|l|}
\hline VARIABLE & Item - Wortlaut \\
\hline Einst_LL1 & $\begin{array}{l}\text { Schülerinnen und Schüler lernen im Unterricht am besten, indem sie } \\
\text { selbst Wege zur Beantwortung von Aufgaben finden. }\end{array}$ \\
\hline Einst_LL2 & $\begin{array}{l}\text { Es ist wichtig für einen Schüler oder eine Schülerin, selbst zu entde- } \\
\text { cken, wie Aufgaben zu bearbeiten sind. }\end{array}$ \\
\hline Einst_LL3 & $\begin{array}{l}\text { Man sollte Schülerinnen und Schülern erlauben, sich eigene Wege } \\
\text { zur Bearbeitung von Aufgaben auszudenken, bevor die Lehrperson } \\
\text { vorführt, wie diese zu beantworten sind. }\end{array}$ \\
\hline Einst_LL4 & $\begin{array}{l}\text { Im Unterricht werden die Lehrziele am besten erreicht, wenn } \\
\text { Schülerinnen und Schüler ihre eigenen Methoden finden, um Aufga- } \\
\text { ben zu bearbeiten. }\end{array}$ \\
\hline Einst_LL5 & $\begin{array}{l}\text { Es hilft Schülerinnen und Schüler Unterrichtsinhalte zu begreifen, } \\
\text { wenn man sie ihre eigenen Ideen diskutieren lässt. }\end{array}$ \\
\hline Einst_LL6 & $\begin{array}{l}\text { Lehrerinnen und Lehrer sollten Schülerinnen und Schüler, die Schwie- } \\
\text { rigkeiten beim Bearbeiten einer Aufgabe haben, erlauben, mit eigenen } \\
\text { Bearbeitungsversuchen fortzufahren. }\end{array}$ \\
\hline Einst_LL7 & $\begin{array}{l}\text { Lehrpersonen sollten Schülerinnen und Schüler auffordern, eigene } \\
\text { Wege zur Aufgabenbearbeitung zu entdecken. }\end{array}$ \\
\hline
\end{tabular}


Skala Einstellungen zum transmissiven Lernen und Lehren - transmissiv SK_L_ einst_LL_Transmissiv

Quelle: Kunter et al., 2017

$\begin{array}{ll}\text { Ausprägung: } & 3 \text { trifft } z u \\ & 2 \text { trifft eher } z u \\ & 1 \text { trifft eher nicht } z u \\ & 0 \text { trifft nicht } z u\end{array}$

Anweisung: Wie sehr stimmen Sie den folgenden Aussagen zu?

\begin{tabular}{|l|l|}
\hline VARIABLE & Item - Wortlaut \\
\hline Einst_LL8 & $\begin{array}{l}\text { Schülerinnen und Schüler lernen am besten, indem sie den Erklärun- } \\
\text { gen der Lehrerin oder des Lehrers folgen. }\end{array}$ \\
\hline Einst_LL9 & $\begin{array}{l}\text { An einem vorgeführten Beispiel lernen die Schülerinnen und Schüler } \\
\text { am besten. }\end{array}$ \\
\hline Einst_LL10 & $\begin{array}{l}\text { Den meisten Schülerinnen und Schüler muss man an einer Reihe von } \\
\text { Beispielen zeigen, wie Aufgaben zu bearbeiten sind. }\end{array}$ \\
\hline Einst_LL11 & $\begin{array}{l}\text { Schülerinnen und Schüler lernen durch die Demonstration von Bei- } \\
\text { spielaufgaben am besten. }\end{array}$ \\
\hline Einst_LL12 & $\begin{array}{l}\text { Um erfolgreich im Unterricht zu sein, müssen Schülerinnen und } \\
\text { Schüler gute Zuhörerinnen und Zuhörer sein. }\end{array}$ \\
\hline Einst_LL13 & $\begin{array}{l}\text { Schülerinnen und Schüler benötigen immer eine ausführliche Anlei- } \\
\text { tung dazu, wie Arbeitsaufträge zu bearbeiten sind. }\end{array}$ \\
\hline Einst_LL14 & $\begin{array}{l}\text { Am besten lernen Schülerinnen und Schüler aus Darstellungen und } \\
\text { Erklärungen ihrer Lehrkraft. }\end{array}$ \\
\hline
\end{tabular}


Skala Einstellungen zum diskursiven Lernen und Lehren SK_L_einst_diskLL

Quelle: Eigenentwicklung in Anlehnung an Osborne et al., 2019

$\begin{array}{ll}\text { Ausprägung: } & 4 \text { stimme } z u \\ & 3 \\ & 2 \\ & 1 \\ & 0 \text { stimme nicht } z u\end{array}$

Anweisung: Die folgenden Items dienen dazu Ihre Lehrerfahrungen und Überzeugungen in Ihrem Fach, mit dem Sie an der Dialogue Fortbildung teilnehmen, zu erfassen. Bitte beurteilen Sie, inwiefern Sie den folgenden Aussagen zustimmen.

\begin{tabular}{|l|l|}
\hline VARIABLE & Item - Wortlaut \\
\hline Einst_diskLL1 & $\begin{array}{l}\text { Nur begabte Schüler sind in der Lage alternative Ideen zu berücksich- } \\
\text { tigen/bedenken/in Erwägung zu ziehen. }\end{array}$ \\
\hline Einst_diskLL2 & $\begin{array}{l}\text { Das Fach XY zielt nicht auf Diskussionen ab; es geht um die richtigen } \\
\text { Antworten. }\end{array}$ \\
\hline Einst_diskLL3 & Im Fach XY gibt es nichts, worüber diskutiert werden kann. \\
\hline Einst_diskLL4 & SuS lernen im Fach XY, indem sie über die Lerninhalte sprechen. \\
\hline Einst_diskLL5 & In meinem Unterricht greife ich Ideen meiner SuS auf. \\
\hline Einst_diskLL6 & $\begin{array}{l}\text { Es ist eine Herausforderung SUS dazu zu bewegen, dass sie Fragens } \\
\text { stellen. }\end{array}$ \\
\hline Einst_diskLL7 & $\begin{array}{l}\text { Für gewöhnlich sind Schülerdiskussionen die zusätzlich benötigte } \\
\text { Zeit wert. }\end{array}$ \\
\hline Einst_diskLL8 & $\begin{array}{l}\text { Durch die Diskussion der eigenen Ideen mit den anderen wird das } \\
\text { Verständnis im Fach XY verbessert. }\end{array}$ \\
\hline Einst_diskLL9 & Es verwirrt Schüler, wenn sie Erklärungen anderer Schüler hören. \\
\hline
\end{tabular}


Skala Fortbildungsqualität - FORT_Act

Quelle: Gröschner et al., 2015

Ausprägung: $\quad 3$ trifft $z u$

2 trifft eher $z u$

1 trifft eher nicht zu

0 trifft nicht $z u$

Anweisung: Bitte beziehen Sie die nachfolgenden Antworten auf die Dialogue Fortbildung.

\begin{tabular}{|l|l|}
\hline VARIABLE & Item - Wortlaut \\
\hline Act01 & In der Fortbildung konnte ich mich aktiv einbringen. \\
\hline Act02 & $\begin{array}{l}\text { In der Fortbildung konnte ich mein fachliches/pädagogisches Wissen } \\
\text { aktiv einbringen. }\end{array}$ \\
\hline Act03 & $\begin{array}{l}\text { In der Fortbildung konnte ich Beispiele aus meinem Unterricht aktiv } \\
\text { einbringen. }\end{array}$ \\
\hline Act04 & $\begin{array}{l}\text { In der Fortbildung konnte ich Beispiele aus dem außerunterricht- } \\
\text { lichen Schulalltag aktiv einbringen. }\end{array}$ \\
\hline
\end{tabular}

Skala Fortbildungsqualität - FORT_Atmo

Quelle: Gröschner et al., 2015

$\begin{array}{ll}\text { Ausprägung: } & 3 \text { trifft } z u \\ & 2 \text { trifft eher } z u \\ & 1 \text { trifft eher nicht } z u \\ & 0 \text { trifft nicht } z u\end{array}$

Anweisung: Bitte beziehen Sie die nachfolgenden Antworten auf die Dialogue Fortbildung.

\begin{tabular}{|l|l|}
\hline VARIABLE & Item - Wortlaut \\
\hline Atmo01 & In der Fortbildung haben wir uns auch kritisch ausgetauscht. \\
\hline Atmo02 & In der Fortbildung erlebte ich eine vertrauensvolle Atmosphäre. \\
\hline Atmo03 & In der Fortbildung herrschte eine wertschätzende Atmosphäre. \\
\hline
\end{tabular}


Skala Fortbildungsqualität - Fort_Con

Quelle: Gröschner et al., 2015

Ausprägung: $\quad 3$ trifft zu

2 trifft eher $z u$

1 trifft eher nicht $z u$

0 trifft nicht $z u$

Anweisung: Bitte beziehen Sie die nachfolgenden Antworten auf die Dialogue Fortbildung.

\begin{tabular}{|l|l|}
\hline VARIABLE & Item - Wortlaut \\
\hline Con01 & $\begin{array}{l}\text { In der Fortbildung wird die Aktivierung der SchülerInnen im Unter- } \\
\text { richt thematisiert. }\end{array}$ \\
\hline Con02 & $\begin{array}{l}\text { In der Fortbildung werden Verständnisprozesse bei SchülerInnen im } \\
\text { Unterricht thematisiert. }\end{array}$ \\
\hline Con03 & Die Fortbildungsinhalte waren fachlich überzeugend. \\
\hline Con04 & Die Fortbildungsinhalte waren verständlich und nachvollziehbar. \\
\hline Con05 & $\begin{array}{l}\text { In der Fortbildung wurden Unterlagen zur Anwendung und Vertie- } \\
\text { fung der Fachinhalte bereitgestellt. }\end{array}$ \\
\hline
\end{tabular}

Skala Fortbildungsqualität - Fort_Coop

Quelle: Gröschner et al., 2015

Ausprägung: 3 trifft zu

2 trifft eher $z u$

1 trifft eher nicht $z u$

0 triff nicht $z u$

Anweisung: Bitte beziehen Sie die nachfolgenden Antworten auf die Dialogue Fortbildung.

\begin{tabular}{|l|l|}
\hline VARIABLE & Item - Wortlaut \\
\hline Coop01 & $\begin{array}{l}\text { In der Fortbildung habe ich im Austausch mit anderen Teilnehmerln- } \\
\text { nen über meinen Unterricht reflektiert. }\end{array}$ \\
\hline Coop02 & $\begin{array}{l}\text { In der Fortbildung habe ich im Austausch mit anderen Teilnehmerln- } \\
\text { nen über meinen Lehreralltag reflektiert. }\end{array}$ \\
\hline Coop03 & $\begin{array}{l}\text { In der Fortbildung nehme ich mich als Mitglied einer Lerngemein- } \\
\text { schaft wahr. }\end{array}$ \\
\hline
\end{tabular}


Skala Fortbildungsqualität - Fort_Fac

Quelle: Item 1-7 aus Gröschner et al., 2015; Item 8 aus Rzejak \& Liypowsky, (o.J.a)

Ausprägung: $\quad 3$ trifft $z u$

2 trifft eher $z u$

1 trifft eher nicht zu

0 trifft nicht $z u$

Anweisung: Bitte beziehen Sie die nachfolgenden Antworten auf die Dialogue Fortbildung.

\begin{tabular}{|l|l|}
\hline VARIABLE & Item - Wortlaut \\
\hline Fac01 & $\begin{array}{l}\text { Der/Die FortbildungsleiterIn moderiert den Austausch unter den } \\
\text { Lehrkräften. }\end{array}$ \\
\hline Fac02 & $\begin{array}{l}\text { Der/Die Fortbildungsleiterln stellt Bezüge zu meinem eigenen Unter- } \\
\text { richt her. }\end{array}$ \\
\hline Fac03 & Der/Die Fortbildungsleiterln stellt Bezüge zu meinem Lehreralltag her. \\
\hline Fac04 & $\begin{array}{l}\text { Der/Die Fortbildungsleiterln gibt konkrete Unterstützung für den } \\
\text { Transfer in meine berufliche Praxis. }\end{array}$ \\
\hline Fac05 & $\begin{array}{l}\text { Der/Die Fortbildungsleiterln achtet auf die Einhaltung von Kommuni- } \\
\text { kationsregeln in der Fortbildung. }\end{array}$ \\
\hline Fac06 & $\begin{array}{l}\text { Der/Die Fortbildungsleiterln sorgt für eine angenehme Atmosphäre in } \\
\text { der Fortbildung. }\end{array}$ \\
\hline Fac07 & $\begin{array}{l}\text { Der/Die Fortbildungsleiterln hilft mir, mich mit den Inhalten der Fort- } \\
\text { bildung vertraut zu machen. }\end{array}$ \\
\hline Fac08 & Der/Die Fortbildungsleiterln geht auf Fragen der Teilnehmerlnnen ein. \\
\hline
\end{tabular}

Skala Fortbildungsqualität - Fort_Org

Quelle: Eigenentwicklung

Ausprägung: $\quad 3$ trifft $z u$

2 trifft eher $z u$

1 trifft eher nicht zu

0 trifft nicht $z u$

Anweisung: Bitte beziehen Sie die nachfolgenden Antworten auf die Dialogue Fortbildung.

\begin{tabular}{|l|l|}
\hline VARIABLE & Item - Wortlaut \\
\hline Orga01 & Die Fortbildung war im Vorfeld gut organisiert. \\
\hline Orga02 & $\begin{array}{l}\text { Die Fortbildungsinhalte passten genau zu meinen Erwartungen/ Be- } \\
\text { dürfnissen. }\end{array}$ \\
\hline Orga03 & $\begin{array}{l}\text { Die Fortbildungsinhalte entsprachen der Programmausschreibung } \\
\text { bzW. -ankündigung. }\end{array}$ \\
\hline Orga04 & Der Aufbau der Fortbildung war zielführend. \\
\hline Orga05 & Die Fortbildung hätte aus meiner Sicht noch länger dauern können. \\
\hline Orga06 & Die Fortbildung hatte genau den richtigen zeitlichen Umfang. \\
\hline Orga07 & $\begin{array}{l}\text { Die Rahmenbedingungen der Fortbildung (Räumlichkeiten, Medien, } \\
\text { etc.) waren angemessen. }\end{array}$ \\
\hline
\end{tabular}


Skala Fortbildungsqualität - FORT_Plan

Quelle: in Anlehnung an Gröschner et al., 2015

Ausprägung: $\quad 3$ trifft zu

2 trifft eher $z u$

1 trifft eher nicht zu

0 trifft nicht $z u$

Anweisung: Bitte beziehen Sie die nachfolgenden Antworten auf die Dialogue Fortbildung.

\begin{tabular}{|l|l|l|}
\hline Skala & VARIABLE & Item - Wortlaut \\
\hline FORT_Plan & Plan01 & $\begin{array}{l}\text { In der Fortbildung werden meine eigenen Unter- } \\
\text { richtsplanungen besprochen. }\end{array}$ \\
\hline
\end{tabular}

Skala Fortbildungsqualität - FORT_Ref

Quelle: Gröschner et al., 2015

$$
\begin{array}{ll}
\text { Ausprägung: } & 3 \text { trifft zu } \\
& 2 \text { trifft eher } z u \\
& 1 \text { trifft eher nicht zu } \\
& 0 \text { trifft nicht } z u
\end{array}
$$

Anweisung: Bitte beziehen Sie die nachfolgenden Antworten auf die Dialogue Fortbildung.

\begin{tabular}{|l|l|}
\hline VARIABLE & Item - Wortlaut \\
\hline Ref01 & In der Fortbildung habe ich über meinen Unterricht reflektiert. \\
\hline Ref02 & $\begin{array}{l}\text { In der Fortbildung habe ich mittels Videoaufzeichnungen über mei- } \\
\text { nen Unterricht reflektiert. }\end{array}$ \\
\hline
\end{tabular}

\section{Skala Fortbildungsqualität - Trans}

Quelle: in Anlehnung an Gröschner et al., 2015

$$
\begin{array}{ll}
\text { Ausprägung: } & 3 \text { trifft } z u \\
& 2 \text { trifft eher } z u \\
& 1 \text { trifft eher nicht } z u \\
& 0 \text { trifft nicht } z u
\end{array}
$$

Anweisung: Bitte beziehen Sie die nachfolgenden Antworten auf die Dialogue Fortbildung.

\begin{tabular}{|l|l|}
\hline VARIABLE & Item - Wortlaut \\
\hline Trans02 & $\begin{array}{l}\text { In der Fortbildung werden konkrete Handlungen bzw. Handlungs- } \\
\text { alternativen besprochen. }\end{array}$ \\
\hline Trans03 & $\begin{array}{l}\text { In der Fortbildung werden mir eigene Routinen im Unterrichtshan- } \\
\text { deln bewusst. }\end{array}$ \\
\hline Trans04 & $\begin{array}{l}\text { In der Fortbildung werden konkrete Bezüge zu meinem eigenen } \\
\text { Unterricht hergestellt. }\end{array}$ \\
\hline Trans11 & $\begin{array}{l}\text { Aus der Fortbildung konnte ich sehr viel Neues für meinen Unterricht } \\
\text { mitnehmen. }\end{array}$ \\
\hline
\end{tabular}


Skala Fortbildungsqualität - Fort_Wirk

Quelle: Rzejak \& Lipowsky (o.J.b)

Ausprägung: $\quad 3$ trifft $z u$

2 trifft eher $z u$

1 trifft eher nicht zu

0 trifft nicht $z u$

Anweisung: Bitte beziehen Sie die nachfolgenden Antworten auf die Dialogue Fortbildung.

\begin{tabular}{|l|l|}
\hline VARIABLE & Item - Wortlaut \\
\hline WirkS01 & $\begin{array}{l}\text { In der Fortbildung wurde ich dazu angeregt, die Lernprozesse von } \\
\text { Schülern konkret nachzuvollziehen. }\end{array}$ \\
\hline WirkS02 & $\begin{array}{l}\text { In der Fortbildung wurde ich dazu angeregt, die Perspektive von } \\
\text { Schülern einzunehmen. }\end{array}$ \\
\hline WirkS03 & $\begin{array}{l}\text { In der Fortbildung habe ich erfahren, wie ich eigene Ideen und Vor- } \\
\text { stellungen der Schüler sichtbar machen kann. }\end{array}$ \\
\hline WirkS04 & $\begin{array}{l}\text { In der Fortbildung wurde deutlich, welche Folgen mein Lehrerhan- } \\
\text { deln für das Lernen der Schüler haben kann. }\end{array}$ \\
\hline WirkS05 & $\begin{array}{l}\text { In der Fortbildung wurden mir die Wirkungen meines unterrichtlichen } \\
\text { Handelns auf das Lernen der Schüler bewusst. }\end{array}$ \\
\hline WirkS06 & $\begin{array}{l}\text { In der Fortbildung wurde mir klar, wie eng Lehrerhandeln und Schü- } \\
\text { lerhandeln miteinander verbunden sind. }\end{array}$ \\
\hline
\end{tabular}

Skala Fortbildungs(nutzungs)motivation Basic needs - MOT_AUT

Quelle: Gröschner et al., 2015, angelehnt an Seidel, Prenzel, \& Kobarg, 2005

Ausprägung: $\quad 3$ trifft $z u$

2 trifft eher $z u$

1 trifft eher nicht zu

0 trifft nicht zu

Anweisung: In den Fortbildungen ...

\begin{tabular}{|l|l|}
\hline VARIABLE & Item - Wortlaut \\
\hline AUT01 & $\ldots$ war der Leiter offen für meine Bedürfnisse. \\
\hline AUT02 & $\begin{array}{l}\text {.. wurde ich ermuntert, selbst zu überlegen, wie man die Inhalte im } \\
\text { Unterricht umsetzt. }\end{array}$ \\
\hline AUT03 & $\ldots$ gab mir der Leiter genügend Möglichkeit, selbständig zu arbeiten. \\
\hline AUT04 & $\begin{array}{l}\ldots \text { standen verschiedene Lösungsmöglichkeiten und Ansätze zur } \\
\text { Diskussion. }\end{array}$ \\
\hline AUT05 & $\ldots$ konnte ich bei der Gestaltung des/der Workshop(s) mitbestimmen. \\
\hline
\end{tabular}


Skala Fortbildungs(nutzungs)motivation Basic needs - MOT_SOZ

Quelle: Gröschner et al., 2015, angelehnt an Seidel et al., 2005

Ausprägung: $\quad 3$ trifft zu

2 trifft eher $z u$

1 trifft eher nicht $z u$

0 triff nicht $z u$

Anweisung: In den Fortbildungen...

\begin{tabular}{|l|l|}
\hline VARIABLE & Item - Wortlaut \\
\hline SOZ_01 & .. empfand ich die Stimmung als angenehm. \\
\hline SOZ_02 & ... haben wir auch gelacht. \\
\hline SOZ_03 & \begin{tabular}{l}
... hatte ich das Gefühl, dass meine Beiträge geschätzt werden. \\
\hline SOZ_04 \\
kann.
\end{tabular} \\
\hline SOZ_05 & $\ldots$ habe ich gelernt, wie ich einen Kollegen/eine Kollegin unterstützen \\
\hline
\end{tabular}

Skala Fortbildungs(nutzungs)motivation Basic needs - MOT_KOM

Quelle: Gröschner et al., 2015, angelehnt an Seidel et al., 2005

Ausprägung: 3 triff zu

2 trifft eher zu

1 trifft eher nicht $z u$

0 trifft nicht $z u$

Anweisung: Wie haben Sie den/die bisherigen Workshop(s) erlebt? Im Workshop ...

\begin{tabular}{|l|l|}
\hline VARIABLE & Item - Wortlaut \\
\hline KOM_01 & ... fühle ich mich persönlich gefördert. \\
\hline KOM_02 & $\ldots$ werden mir auch schwierige Aufgaben zugetraut. \\
\hline KOM_03 & $\ldots$ kann ich mein Wissen einbringen. \\
\hline KOM_04 & $\ldots$ habe ich schon viel gelernt. \\
\hline KOM_05 & ... habe ich von den Kolleginnen und Kollegen viel gelernt. \\
\hline
\end{tabular}


Skala Zufriedenheit mit der Fortbildung - FORT_ZUF

Quelle: Gröschner et al., 2015

$\begin{array}{ll}\text { Ausprägung: } & 9 \text { sehr zufrieden } \\ & 8 \\ 7 \\ 6 \\ 5 \\ 4 \\ 3 \\ 2 \\ 1 \\ \text { 0 sehr unzufrieden }\end{array}$

\begin{tabular}{|l|l|}
\hline VARIABLE & Item - Wortlaut \\
\hline ZUF01 & $\begin{array}{l}\text { Wie zufrieden sind Sie mit dem Verlauf der Fortbildung? Bitte setzen } \\
\text { Sie ein Kreuz. }\end{array}$ \\
\hline
\end{tabular}

Skala Fortbildungstransfer - FORT_Trans

Quelle: Rzejak et al., 2014

$\begin{array}{ll}\text { Ausprägung: } & 5 \text { trifft voll und ganz zu } \\ & 4 \text { trifft voll zu } \\ & 3 \text { trifft eher voll zu } \\ & 2 \text { trifft eher nicht } z u \\ & 1 \text { trifft eher gar nicht } z u \\ & 0 \text { trifft gar nicht } z u\end{array}$

Anweisung: Für die Fortbildung kann ich zum jetzigen Zeitpunkt sagen:

\begin{tabular}{|l|l|}
\hline VARIABLE & Item - Wortlaut \\
\hline Trans01 & Ich habe die Fortbildung als sehr hilfreich erlebt. \\
\hline Trans05 & $\begin{array}{l}\text { Ich habe die Anregungen aus der Fortbildung anschließend im Unter- } \\
\text { richt umgesetzt. }\end{array}$ \\
\hline Trans06 & Die Fortbildung hat einen positiven Einfluss auf meinen Unterricht. \\
\hline Trans07 & $\begin{array}{l}\text { Die Differenz zwischen den Inhalten der Fortbildung und der tatsäch- } \\
\text { lichen Unterrichtswirklichkeit ist zu groß. }\end{array}$ \\
\hline Trans08 & $\begin{array}{l}\text { Die Fortbildung hat mir bei der Unterrichtsplanung und } \\
\text {-durchführung geholfen. }\end{array}$ \\
\hline Trans09 & $\begin{array}{l}\text { Die Anregungen aus der Fortbildung lässt sich gut in den Unterricht } \\
\text { übertragen. }\end{array}$ \\
\hline Trans10 & Für mich hat sich die Teilnahme an der Fortbildung gelohnt. \\
\hline
\end{tabular}




\subsection{Befragung der Lehrenden über deren situationale Wahrnehmung der videographierten Unterrichtsstunde}

Im Rahmen der Videographie wurden, angelehnt an bisherige Studien (Seidel et al., 2005), die Lehrpersonen im Anschluss an die Stunde nach der Wahrnehmung des Ablaufs der Unterrichtsstunde im Vergleich zum sonstigen Unterricht befragt.

Skala typische Unterrichtsstunde - SK_L_typisch

Quelle: Seidel, Dalehefte, Lehrke, \& Trepke, 2005

Anweisung: Wie würden Sie die aufgezeichnete (Doppel-)Stunde als Ganzes beschreiben? War sie typisch für die Stunden, die Sie normalerweise unterrichten?

\begin{tabular}{|l|l|}
\hline VARIABLE & Item - Wortlaut \\
\hline Typisch1 & Absolut untypisch \\
\cline { 2 - 2 } & Nicht typisch \\
\cline { 2 - 2 } & Größtenteils typisch \\
\cline { 2 - 2 } & Sehr typisch \\
\hline
\end{tabular}

Skala untypische Unterrichtsstunde - SK_L_untypisch

Quelle: Seidel, Dalehefte et al., 2005

\begin{tabular}{|l|l|}
\hline VARIABLE & Item - Wortlaut \\
\hline Untypisch1 & Was war - gegebenenfalls - untypisch? \\
\hline
\end{tabular}

Skala Selbsteinschätzung Verhalten - SK_L_Verhalten

Quelle: Seidel, Dalehefte et al., 2005

Anweisung: Wie würden Sie das Verhalten Ihrer Schülerinnen und Schüler in der aufgezeichneten (Doppel-)Stunde beschreiben?

\begin{tabular}{|l|l|}
\hline VARIABLE & Item - Wortlaut \\
\hline Verhalten1 & sehr anders als ihr übliches Verhalten \\
\cline { 2 - 2 } & etwas anders als ihr übliches Verhalten \\
\cline { 2 - 2 } & ähnlich zu ihrem üblichen Verhalten \\
\cline { 2 - 2 } & sehr ähnlich zu ihrem üblichen Verhalten \\
\hline
\end{tabular}


Skala empfundene Schülerbeteiligung SK_L_empfSbtlg

Quelle: Eigenentwicklung

Anweisung: Bitte geben Sie an:

\begin{tabular}{|l|l|}
\hline VARIABLE & Item - Wortlaut \\
\hline EmpfSbtlg1 & Mit wie vielen Schülern haben Sie heute interagiert? \\
\hline EmpfSbtlg2 & Wie vielen Schülern haben Sie heute Feedback gegeben? \\
\hline EmpfSbtlg3 & Wie viele Schüler haben sich heute durchschnittlich gemeldet? \\
\hline EmpfSbtlg4 & Mit welchem Schüler haben Sie heute am meisten verbal interagiert? \\
\hline
\end{tabular}

\section{3 „Track your Fortbildung“ - Online Tool zur Prozessbegleitung der Fortbildung}

Entlang des Anspruchs der proximalen Instrumentenentwicklung für den vorliegenden Fortbildungskontext, sowie der Frage, ob die Lehrkräfte die erlernten Fortbildungsinhalte auch in anderen Klassen zum Einsatz bringen würden, wurde der Online-Kurzfragenbogen Track your Fortbildung entwickelt. Die Lehrpersonen wurden dazu aufgefordert, alle 2 Wochen diesen kurzen Fragebogen online zu bearbeiten und somit den Transfer von Fortbildungsinhalten in ihren Unterricht zu dokumentieren.

Skala Transfer spezifischer Inhalte - SK_L_transfer

Quelle: Eigenentwicklung, in Anlehnung an Osborne et al., 2019

Ausprägung: 5 trifft voll $z u$

4

3

2

1

0 trifft gar nicht $z u$

Anweisung: Seit der letzten Dialogue Fortbildung gelang es mir in meinem Unterricht ...

\begin{tabular}{|l|l|}
\hline VARIABLE & Item - Wortlaut \\
\hline Transfer1 & .. Schüleraktivierende Methoden umzusetzen. \\
\hline Transfer2 & $\begin{array}{l}\text {.. Gelegenheiten für Schüler-zu-Schüler-Gespräche mit abwechseln- } \\
\text { den Diskussionsformaten zu schaffen (ganze Klasse, kleine Gruppen, } \\
\text { Partnergespräche). }\end{array}$ \\
\hline Transfer3 & $\begin{array}{l}\text {.. mit vielen verschiedenen Schülern im Unterrichtsgespräch zu inter- } \\
\text { agieren. }\end{array}$ \\
\hline Transfer4 & $\begin{array}{l}\text {.. offene Fragen zu stellen, welche verschiedene Schülerantworten } \\
\text { zulassen. }\end{array}$ \\
\hline Transfer5 & ... meine Schüler zu bekräftigen, sich am Gespräch zu beteiligen. \\
\hline Transfer6 & $\ldots$ meinen Schülern Hinweise für ihr weiteres Lernen zu geben. \\
\hline Transfer7 & ... mit Fehlern der Schüler konstruktiv umzugehen. \\
\hline Transfer8 & .. Lernziele transparent zu machen. \\
\hline
\end{tabular}




\section{Alexander Gröschner, Ann-Kathrin Schindler, Maralena Weil, Ricardo Böheim, Maximilian Knogler \& Tina Seidel}

\section{Instrumente zur Befragung von Lernenden}

Entlang des in der Einleitung vorgestellten Modells von Clarke und Hollingsworth (2002) stellte die Erfassung von Schülerwahrnehmungen des Unterrichts ein zentrales Anliegen der Dialogue Studie dar. Individuelle Voraussetzungen der Schülerinnen und Schüler zu erfassen war u.a. auch von Bedeutung, um differenzielle Wirkungen der Fortbildungen auf die Schülerlernprozesse untersuchen zu können.

Die eingesetzten Skalen und Items stammen zum Teil aus anderen Studien, wie z.B. aus BilWiss (Kunter et al., 2017) oder der IPN-Videostudie (Seidel et al., 2003). Wie zu Beginn des Buches beschrieben, war es zudem Ziel auch auf Schülerseite stärker proximale Erhebungsinstrumente entlang des Fortbildungsschwerpunkts einzusetzen. Vereinzelte Skalen und Items wurden daher im Rahmen des Projekts neu oder in Anlehnung an ähnliche Forschungsprojekte (z.B. die PRACTISEStudie von Osborne und Kollegen, 2019) konzipiert. Abbildung 6.1 zeigt die Erhebungszeitpunkte aufgeschlüsselt nach den Fortbildungsangeboten im Verlauf des Schuljahres auf Seiten der Lernenden.

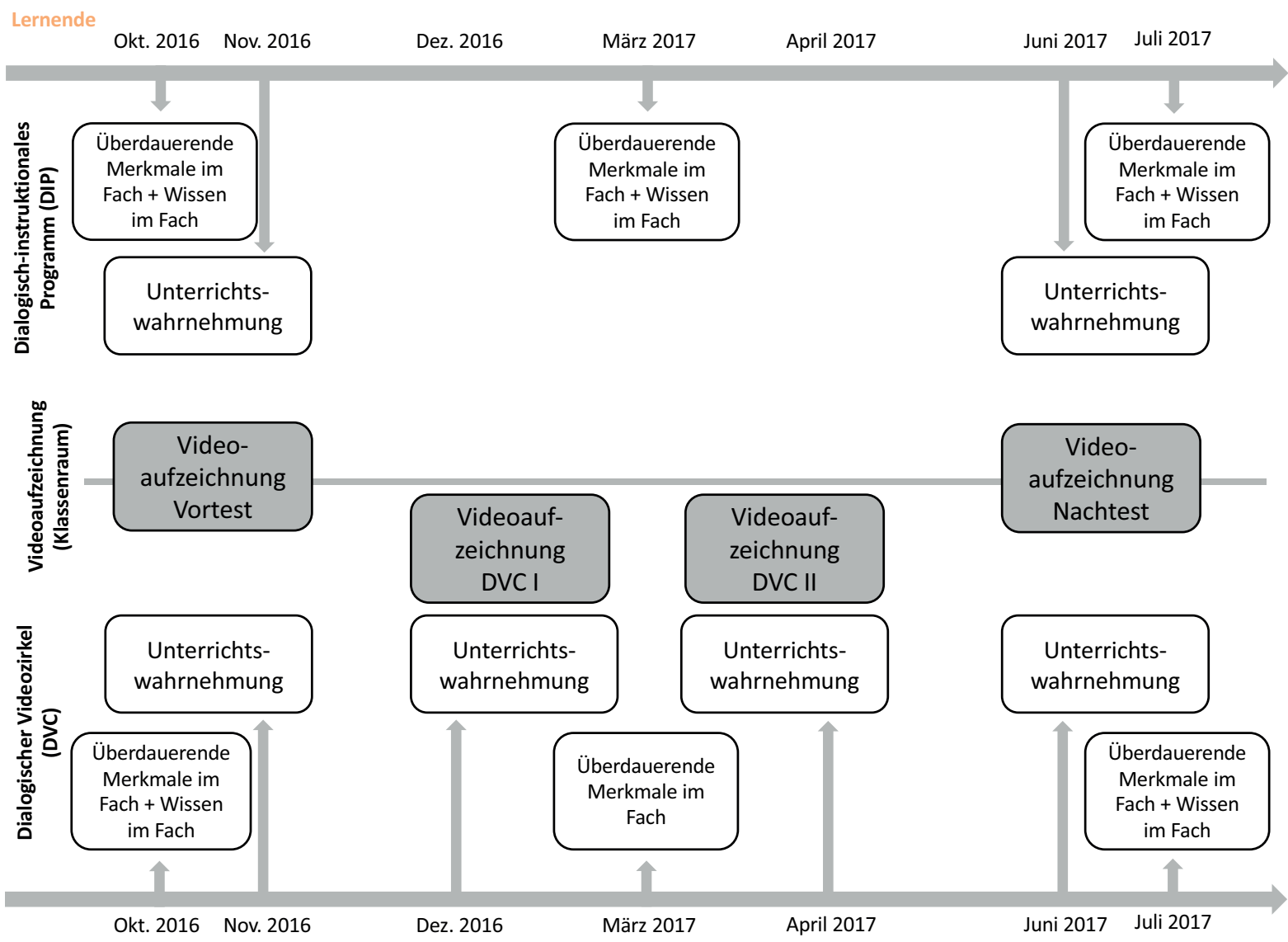

Abbildung 6.1: Übersicht der Erhebungszeitpunkte bei Lehrkräften beider Fortbildungsangebote 


\subsection{Befragung der Lernenden zu überdauernden Merkmalen}

Skala Individuelles Interesse am Unterrichtsfach SK_int

Item 1, 3, 4 adaptiert von Seidel, Prenzel, \& Kobarg, 2005; Item 2, 5, 6, 7, 8, 9 adaptiert von Rakoczy, Buff, \& Klieme, 2005

\section{Ausprägung: 3 stimme vollkommen zu \\ 2 stimme eher $z u$ \\ 1 stimme eher nicht $z u$ \\ 0 stimme gar nicht $z u$}

Anweisung: Wie sehr stimmst du mit den folgenden Aussagen überein?

\begin{tabular}{|l|l|l|l|}
\hline Skala & ALPHA $_{\text {MZP01 }}$ & ALPHA $_{\text {MZP03 }}$ & ALPHA $_{\text {MZP05 }}$ \\
\hline SK_int & .92 & .90 & .92 \\
\hline
\end{tabular}

\begin{tabular}{|l|l|}
\hline VARIABLE & Item - Wortlaut \\
\hline Int1 & Das Fach gefällt mir. \\
\hline Int2 & Das Fach ist spannend. \\
\hline Int3 & Ich beschäftige mich gerne mit dem Fach $x x$. \\
\hline Int4 & Ich eigne mir gerne neues Wissen im Fach xx an. \\
\hline Int5 & Das Fach $x x$ ist mir persönlich wichtig. \\
\hline Int6 & Das Fach $x x$ ist nützlich für mich. \\
\hline Int7 & Das Fach $x x$ ist langweilig. \\
\hline Int8 & Wenn ich ehrlich bin, ist mir das Fach xx gleichgültig. \\
\hline Int9 & Freiwillig würde ich mich nie mit dem Fach xx beschäftigen. \\
\hline
\end{tabular}

Skala Fähigkeitsselbstkonzept im Unterrichtsfach SK_fsk

Quelle: Marsh, Trautwein, Ludtke, Koller, \& Baumert, 2005

$\begin{array}{ll}\text { Ausprägung: } & 3 \text { stimmt genau } \\ & 2 \text { stimmt eher } \\ & 1 \text { stimmt eher nicht } \\ & 0 \text { stimmt gar nicht }\end{array}$

Anweisung: Wie würdest du dich selbst anhand folgender Aussagen einschätzen?

\begin{tabular}{|l|l|l|l|}
\hline Skala & ALPHA $_{\text {MZP01 }}$ & ALPHA $_{\text {MZP03 }}$ & ALPHA $_{\text {MZP05 }}$ \\
\hline SK_fsk & .91 & .90 & .91 \\
\hline
\end{tabular}

\begin{tabular}{|l|l|}
\hline VARIABLE & Item - Wortlaut \\
\hline Fsk1 & Für Unterrichtsfach habe ich einfach keine Begabung. \\
\hline Fsk2 & Unterrichtsfach liegt mir nicht besonders. \\
\hline Fsk3 & Ich bin gut im Unterrichtsfach. \\
\hline Fsk4 & Unterrichtsfach fällt mir leicht. \\
\hline Fsk5 & Mit Unterrichtsfachaufgaben habe ich immer Probleme. \\
\hline
\end{tabular}




\section{Skala Selbstwirksamkeit im Unterrichtsfach SK_sw}

Quelle: Ramm, Prenzel, Baumert, Blum, Lehmann, Leutner, Neubrand, Pekrun, Rolff, Rost, \& Schiefele, 2006

$\begin{array}{ll}\text { Ausprägung: } & 3 \text { trifft } z u \\ & 2 \text { trifft eher } z u \\ & 1 \text { trifft eher nicht } z u \\ & 0 \text { trifft nicht } z u\end{array}$

Anweisung: Wie würdest du dich selbst anhand folgender Aussagen einschätzen?

\begin{tabular}{|l|l|l|l|}
\hline Skala & ALPHA $_{\text {MZP01 }}$ & ALPHA $_{\text {MZP03 }}$ & ALPHA $_{\text {MZP05 }}$ \\
\hline SK_sw & .85 & .86 & .91 \\
\hline
\end{tabular}

\begin{tabular}{|l|l|}
\hline VARIABLE & Item - Wortlaut \\
\hline Sw1 & $\begin{array}{l}\text { In Unterrichtsfach bin ich sicher, dass ich auch den schwierigsten Stoff } \\
\text { verstehen kann. }\end{array}$ \\
\hline Sw2 & $\begin{array}{l}\text { Ich bin überzeugt, dass ich auch den kompliziertesten Stoff, den der } \\
\text { Lehrer im Unterrichtsfach vorstellt, verstehen kann. }\end{array}$ \\
\hline Sw3 & $\begin{array}{l}\text { Ich bin überzeugt, dass ich in Hausaufgaben und Prüfungen in Unter- } \\
\text { richtsfach gute Leistungen erzielen kann. }\end{array}$ \\
\hline Sw4 & $\begin{array}{l}\text { Ich bin überzeugt, dass ich die Fertigkeiten, die in Unterrichtsfach } \\
\text { unterrichtet werden beherrschen kann. }\end{array}$ \\
\hline
\end{tabular}

Skala Lernstrategien - Elaboration SK_ela

Quelle: Ramm et al., 2006

$\begin{array}{ll}\text { Ausprägung: } & 3 \text { trifft } z u \\ & 2 \text { trifft eher } z u \\ & 1 \text { trifft eher nicht zu } \\ & 0 \text { trifft nicht } z u\end{array}$

Anweisung: Wie würdest du dich selbst anhand folgender Aussagen einschätzen?

\begin{tabular}{|l|l|l|l|}
\hline Skala & ALPHA $_{\text {MZP01 }}$ & ALPHA $_{\text {MZP03 }}$ & ALPHA $_{\text {MZP05 }}$ \\
\hline SK_ela & .73 & .77 & .80 \\
\hline
\end{tabular}

\begin{tabular}{|l|l|}
\hline VARIABLE & Item - Wortlaut \\
\hline Ela1 & Bei Unterrichtsfachaufgaben überlege ich mir oft neue Lösungswege. \\
\hline Ela2 & $\begin{array}{l}\text { Ich überlege mir, wie das, was ich in Unterrichtsfach gelernt habe, im } \\
\text { Alltag angewendet werden kann. }\end{array}$ \\
\hline Ela3 & $\begin{array}{l}\text { Neues in Unterrichtsfach versuche ich besser zu verstehen, indem ich } \\
\text { Verbindungen zu Dingen herstelle, die ich schon kenne. }\end{array}$ \\
\hline Ela4 & $\begin{array}{l}\text { Wenn ich eine Unterrichtsfachaufgabe löse, überlege ich oft, wie die } \\
\text { kösung für andere interessante Fragestellungen verwendet werden } \\
\text { könnte. }\end{array}$ \\
\hline Ela5 & $\begin{array}{l}\text { Wenn ich für Unterrichtsfach lerne, versuche ich den Stoff mit Dingen } \\
\text { zu verbinden, die ich in anderen Fächern gelernt habe. }\end{array}$ \\
\hline
\end{tabular}




\subsection{Befragung der Lernenden zur situationalen Wahrnehmung}

Im Rahmen der Videographie wurden, angelehnt an bisherige Studien (Seidel et al., 2005), die Lernenden im Anschluss an die Stunde nach deren Wahrnehmung der Unterrichtsstunde befragt.

Skala Nachvollziehende Elaboration SK_n_ela

Quelle: Seidel, Prenzel, Duit, \& Lehrke, 2003

Ausprägung: 3 trifft $z u$

2 trifft eher $z u$

1 trifft eher nicht $z u$

0 trifft nicht $z u$

Anweisung: In der vorangegangenen Unterrichtsstunde ...

\begin{tabular}{|l|l|l|l|l|}
\hline Skala & ALPHA $_{\text {MZP01 }}$ & ALPHA $_{\text {MZP02 }}$ & ALPHA $_{\text {MZP04 }}$ & ALPHA $_{\text {MZP05 }}$ \\
\hline SK_n_ela & .79 & .87 & .88 & .86 \\
\hline
\end{tabular}

\begin{tabular}{|l|l|}
\hline VARIABLE & Item - Wortlaut \\
\hline n_ela1 & $\begin{array}{l}\ldots \text { hatte ich das Gefühl, die einzelnen Schritte gut nachvollziehen zu } \\
\text { können. }\end{array}$ \\
\hline n_ela2 & $\ldots$ waren mir die wesentlichen Dinge klar. \\
\hline n_ela3 & $\ldots$ bin ich die ganze Zeit über gut mitgekommen. \\
\hline n_ela4 & $\ldots$ war mir klar, was die neuen Begriffe bedeuten. \\
\hline
\end{tabular}

Skala Vertiefende Elaboration SK_v_ela

Quelle: Seidel et al., 2003

$\begin{array}{ll}\text { Ausprägung: } & 3 \text { trifft } z u \\ & 2 \text { trifft } \text { eher } z u \\ & 1 \text { trifft eher nicht } z u \\ & 0 \text { trifft nicht } z u\end{array}$

Anweisung: In der vorangegangenen Unterrichtsstunde ...

\begin{tabular}{|l|l|l|l|l|}
\hline Skala & ALPHA $_{\text {MZP01 }}$ & ALPHA $_{\text {MZP02 }}$ & ALPHA $_{\text {MZP04 }}$ & ALPHA $_{\text {MZP05 }}$ \\
\hline SK_v_ela & .58 & .61 & .70 & .64 \\
\hline
\end{tabular}

\begin{tabular}{|l|l|}
\hline VARIABLE & Item - Wortlaut \\
\hline v_ela1 & $\ldots$ habe ich die Inhalte in eigene Worte gefasst. \\
\hline v_ela2 & $\ldots$ sind mir viele Ideen zum Thema durch den Kopf gegangen. \\
\hline v_ela3 & $\ldots$ habe ich mir die Inhalte an Beispielen vorgestellt. \\
\hline v_ela4 & $\ldots$ habe ich den Stoff auf andere Aufgaben/ Beispiele angewendet. \\
\hline
\end{tabular}


Skala Organisierende Prozesse SK_org_p

Quelle: Seidel et al., 2003

Ausprägung: $\quad 3$ trifft zu

2 trifft eher $z u$

1 trifft eher nicht $z u$

0 trifft nicht zu

Anweisung: In der vorangegangenen Unterrichtsstunde ...

\begin{tabular}{|l|l|l|l|l|}
\hline Skala & ALPHA $_{\text {MZP01 }}$ & ALPHA $_{\text {MZP02 }}$ & ALPHA $_{\text {MZP04 }}$ & ALPHA $_{\text {MZP05 }}$ \\
\hline SK_org_p & .63 & .74 & .75 & .75 \\
\hline
\end{tabular}

\begin{tabular}{|l|l|}
\hline VARIABLE & Item - Wortlaut \\
\hline Org_p1 & $\begin{array}{l}\text {.. habe ich darüber nachgedacht, wie die Dinge im Einzelnen zusam- } \\
\text { menhängen. }\end{array}$ \\
\hline Org_p2 & $\ldots$ habe ich versucht, Zusammenhänge zu sehen. \\
\hline Org_p3 & $\ldots$ habe ich mir in Gedanken Abläufe vorgestellt. \\
\hline Org_p4 & $\begin{array}{l}\text {... war mir klar, was bei diesem Thema besonders wichtig und was } \\
\text { eher unwichtig ist. }\end{array}$ \\
\hline Org_p5 & $\ldots$ habe ich in Gedanken das Wichtigste selbst zusammengefasst. \\
\hline Org_p6 & $\ldots$ wusste ich, wie die Inhalte aufeinander aufbauen. \\
\hline
\end{tabular}

Skala Situationales Interessse - Catch SK_sit_ic

Quelle: Eigenentwicklung, adaptiert von Knogler, Harackiewicz, Gegenfurtner, \& Lewalter, 2015

Ausprägung: $\quad 3$ trifft zu

2 trifft eher $z u$

1 trifft eher nicht $z u$

0 trifft nicht zu

Anweisung: In der vorangegangenen Unterrichtsstunde ...

\begin{tabular}{|l|l|l|l|l|}
\hline Skala & ALPHA $_{\text {MZP01 }}$ & ALPHA $_{\text {MZP02 }}$ & ALPHA $_{\text {MZP04 }}$ & ALPHA $_{\text {MZP05 }}$ \\
\hline SK_sit_ic & .86 & .88 & .88 & .89 \\
\hline
\end{tabular}

\begin{tabular}{|l|l|}
\hline VARIABLE & Item - Wortlaut \\
\hline Sit_ic1 & $\ldots$ hat der Unterricht meine Neugier geweckt. \\
\hline Sit_ic2 & $\ldots$ hat der Unterricht meine Aufmerksamkeit gefesselt. \\
\hline Sit_ic3 & $\ldots$ war ich auf den Unterricht konzentriert. \\
\hline Sit_ic4 & $\ldots$ war der Unterricht für mich unterhaltsam. \\
\hline Sit_ic5 & $\ldots$ hat mir der Unterricht Spaß gemacht. \\
\hline Sit_ic6 & $\ldots$ war der Unterricht für mich spannend. \\
\hline
\end{tabular}


Skala Situationales Interessse - Hold SK_sit_ih

Quelle: Eigenentwicklung, adaptiert von Knogler et al., 2015

$\begin{array}{ll}\text { Ausprägung: } & 3 \text { sehr } \\ & 2 \text { ziemlich } \\ & 1 \text { etwas } \\ & 0 \text { gar nicht }\end{array}$

Anweisung: Wenn du an die heutige Mathematikstunde denkst...

\begin{tabular}{|l|l|l|l|l|}
\hline Skala & ALPHA $_{\text {MZP01 }}$ & ALPHA $_{\text {MZP02 }}$ & ALPHA $_{\text {MZP04 }}$ & ALPHA $_{\text {MZP05 }}$ \\
\hline SK_sit_ih & .79 & .86 & .87 & .84 \\
\hline
\end{tabular}

\begin{tabular}{|c|c|}
\hline VARIABLE & Item - Wortlaut \\
\hline Sit_ih1 & $\begin{array}{l}\text {... möchtest du dich über die Inhalte des Unterrichts mit anderen } \\
\text { unterhalten. }\end{array}$ \\
\hline Sit_ih2 & $\begin{array}{l}\text {... bist du auf Themen gestoßen, zu denen du gerne mehr Informatio- } \\
\text { nen hättest. }\end{array}$ \\
\hline Sit_ih3 & ... möchtest du über die Inhalte des Unterrichts mehr erfahren. \\
\hline Sit_ih4 & $\begin{array}{l}\text {... war die Beschäftigung mit den Inhalten des Unterrichts für dich } \\
\text { nützlich. }\end{array}$ \\
\hline Sit_ih5 & ... fandest du die Inhalte des Unterrichts für dich bedeutsam. \\
\hline Sit_ih6 & $\begin{array}{l}\text {... war die Auseinandersetzung mit den Inhalten des Unterrichts für } \\
\text { dich wichtig. }\end{array}$ \\
\hline
\end{tabular}

\section{Skala Autonomiegewährung SK_auton}

Quellen: Item 1-4 Seidel et al., 2003; Item 5-8 Ramm et al., 2006

$$
\begin{array}{ll}
\text { Ausprägung: } & 3 \text { trifft } z u \\
& 2 \text { trifft eher } z u \\
& 1 \text { trifft eher nicht zu } \\
& 0 \text { trifft nicht } z u
\end{array}
$$

Anweisung: In der vorangegangenen Unterrichtsstunde ...

\begin{tabular}{|l|l|l|l|l|}
\hline Skala & ALPHA $_{\text {MZP01 }}$ & ALPHA $_{\text {MZP02 }}$ & ALPHA $_{\text {MZP04 }}$ & ALPHA $_{\text {MZP05 }}$ \\
\hline SK_auton & .78 & .72 & .76 & .79 \\
\hline
\end{tabular}

\begin{tabular}{|l|l|}
\hline VARIABLE & Item - Wortlaut \\
\hline Auton1 & $\begin{array}{l}\text {... hatte ich das Gefühl, dass die Lehrerin/ der Lehrer offen war für } \\
\text { unterschiedliche Antworten der Schüler. }\end{array}$ \\
\hline Auton2 & $\begin{array}{l}\text {... wurden wir nach meiner Ansicht von der Lehrerin/ vom Lehrer er- } \\
\text { muntert, selbst zu überlegen, wie man am besten vorgeht. }\end{array}$ \\
\hline Auton3 & $\begin{array}{l}\text {... hatte ich das Gefühl, dass mir die Lehrerin/ der Lehrer genügend } \\
\text { Möglichkeit gab, selbstständig zu arbeiten. }\end{array}$ \\
\hline Auton4 & $\begin{array}{l}\text {.. standen meiner Meinung nach verschiedene Lösungsmöglichkeiten } \\
\text { zur Diskussion. }\end{array}$ \\
\hline Auton5 & $\begin{array}{l}\text {.. hatte ich die Möglichkeit, ein neues Stoffgebiet eigenständig zu be- } \\
\text { arbeiten. }\end{array}$ \\
\hline Auton6 & $\ldots$ konnte ich bei der Unterrichtsgestaltung mitbestimmen. \\
\hline Auton7 & $\ldots$ war es mir möglich, meine Zeit selbst einzuteilen. \\
\hline Auton8 & $\ldots$ hatte ich das Gefühl, dass ich eigene Entscheidungen treffen kann. \\
\hline
\end{tabular}


Skala Kompetenzunterstützung SK_komp

Quellen: Item 1-3 Seidel et al., 2003; Item 4-7 adaptiert nach Ramm et al., 2006

Ausprägung: $\quad 3$ trifft zu

2 trifft eher $z u$

1 trifft eher nicht zu

0 trifft nicht $z u$

Anweisung: In der vorangegangenen Unterrichtsstunde ...

\begin{tabular}{|l|l|l|l|l|}
\hline Skala & ALPHA $_{\text {MZP01 }}$ & ALPHA $_{\text {MZP02 }}$ & ALPHA $_{\text {MZP04 }}$ & ALPHA $_{\text {MZP05 }}$ \\
\hline SK_komp & .73 & .73 & .76 & .81 \\
\hline
\end{tabular}

\begin{tabular}{|l|l|}
\hline VARIABLE & Item - Wortlaut \\
\hline Komp1 & $\begin{array}{l}\text {.. hat uns die Lehrerin/der Lehrer meiner Ansicht nach auch an- } \\
\text { spruchsvolle Dinge zugetraut. }\end{array}$ \\
\hline Komp2 & $\begin{array}{l}\text {.. hat uns die Lehrerin/der Lehrer meiner Meinung nach zugetraut, } \\
\text { dass wir selbstständig arbeiten. }\end{array}$ \\
\hline Komp3 & $\begin{array}{l}\text {. bin ich der Meinung, konnte ich Hilfe bekommen, falls etwas zu } \\
\text { schwer wurde. }\end{array}$ \\
\hline Komp4 & $\ldots$ fühlte ich mich persönlich gefördert. \\
\hline Komp5 & $\begin{array}{l}\text {.. hatte ich das Gefühl, es wurden mir auch schwierige Aufgaben } \\
\text { zugetraut. }\end{array}$ \\
\hline Komp6 & $\begin{array}{l}\text {. konnte ich mein Wissen meiner Ansicht nach in den Unterricht } \\
\text { einbringen. }\end{array}$ \\
\hline Komp7 & $\begin{array}{l}\text {.. habe ich bei diesem Lehrer/bei dieser Lehrerin meiner Meinung } \\
\text { nach viel gelernt. }\end{array}$ \\
\hline
\end{tabular}

Skala Beziehung zur Lehrerperson SK_bezlp

Quelle: Eigenentwicklung

$\begin{array}{ll}\text { Ausprägung: } & 3 \text { trifft zu } \\ & 2 \text { trifft eher } z u \\ & 1 \text { trifft eher nicht } z u \\ & 0 \text { trifft nicht } z u\end{array}$

Anweisung: Wenn ich an meine Lehrerin/meinen Lehrer denke ...

\begin{tabular}{|l|l|l|l|l|}
\hline Skala & ALPHA $_{\text {MZP01 }}$ & ALPHA $_{\text {MZP02 }}$ & ALPHA $_{\text {MZP04 }}$ & ALPHA $_{\text {MZP05 }}$ \\
\hline SK_bezlp & .73 & .78 & .77 & .85 \\
\hline
\end{tabular}

\begin{tabular}{|l|l|}
\hline VARIABLE & Item - Wortlaut \\
\hline Bezlp1 & .. habe ich das Gefühl, dass sie/er mich mag. \\
\hline Bezlp2 & \begin{tabular}{l}
.. habe ich das Gefühl, dass sie/er mich wahrnimmt. \\
\hline Bezlp3
\end{tabular} \\
$\begin{array}{l}\text {.. habe ich das Gefühl, dass sie/er meine Beteiligung am Unterricht } \\
\text { schätzt. }\end{array}$ \\
\hline
\end{tabular}


Skala Bereitschaft und Möglichkeit für Beteiligung SK_betlg

Quelle: Eigenentwicklung

$\begin{array}{ll}\text { Ausprägung: } & \text { 4: } 10 \mathrm{mal} \text { und mehr } \\ & \text { 3: 7-9 mal } \\ & \text { 2: } 4-6 \mathrm{mal} \\ & \text { 1: } 1-3 \mathrm{mal} \\ & \text { 0: } 0 \mathrm{mal}\end{array}$

Anweisung: Wie oft hast du dich heute am Unterrichtsgespräch beteiligt?

\begin{tabular}{|l|l|l|l|l|}
\hline Skala & ALPHA $_{\text {MZP01 }}$ & ALPHA $_{\text {MZP02 }}$ & ALPHA $_{\text {MZP04 }}$ & ALPHA $_{\text {MZP05 }}$ \\
\hline SK_betlg & .71 & .71 & .60 & .71 \\
\hline
\end{tabular}

\begin{tabular}{|l|l|}
\hline VARIABLE & Item - Wortlaut \\
\hline Betlg1 & Ich habe mich gemeldet. \\
\hline Betlg2 & Ich habe mich verbal aktiv beteiligt. \\
\hline Betlg3 & Ich wollte mich beteiligen, durfte aber nicht. \\
\hline
\end{tabular}

Skala Aktivierung - Interaktionsverhalten SK_aktiv

Quelle: Eigenentwicklung

$\begin{array}{ll}\text { Ausprägung: } & 3 \text { trifft } z u \\ & 2 \text { trifft eher } z u \\ & 1 \text { trifft eher nicht } z u \\ & 0 \text { trifft nicht } z u\end{array}$

Anweisung: Wenn du an das heutige Unterrichtsgespräch denkst, wie hat der/die Lehrer/in mit euch interagiert?

\begin{tabular}{|l|l|l|l|l|}
\hline Skala & ALPHA $_{\text {MZP01 }}$ & ALPHA $_{\text {MZP02 }}$ & ALPHA $_{\text {MZP04 }}$ & ALPHA $_{\text {MZP05 }}$ \\
\hline SK_aktiv & .70 & .83 & .82 & .78 \\
\hline
\end{tabular}

\begin{tabular}{|l|l|}
\hline VARIABLE & Item - Wortlaut \\
\hline Aktiv1 & $\begin{array}{l}\text { Die Lehrerin/der Lehrer hat heute nur mit wenigen Schülern in der } \\
\text { Klasse interagiert. }\end{array}$ \\
\hline Aktiv2 & $\begin{array}{l}\text { Die Lehrerin/der Lehrer hat heute immer mit den gleichen Schülern } \\
\text { interagiert. }\end{array}$ \\
\hline Aktiv3 & $\begin{array}{l}\text { Die Lehrerin/der Lehrer hat heute mit denjenigen interagiert, die sie } \\
\text { sonst auch immer dran nimmt. }\end{array}$ \\
\hline
\end{tabular}


Skala Aktivierung - Regeln und Verantwortlichkeiten SK_aktrv

Quelle: Eigenentwicklung

Ausprägung: $\quad 3$ trifft zu

2 trifft eher $z u$

1 trifft eher nicht zu

0 trifft nicht $z u$

Anweisung: Wenn du an das heutige Unterrichtsgespräch denkst, wie hat der/die Lehrer/in mit euch interagiert?

\begin{tabular}{|l|l|l|l|l|}
\hline Skala & ALPHA $_{\text {MZP01 }}$ & ALPHA $_{\text {MZP02 }}$ & ALPHA $_{\text {MZP04 }}$ & ALPHA $_{\text {MZP05 }}$ \\
\hline SK_aktrv & .63 & .53 & .64 & .72 \\
\hline
\end{tabular}

\begin{tabular}{|l|l|}
\hline VARIABLE & Item - Wortlaut \\
\hline Aktrv1 & $\begin{array}{l}\text { Die Lehrerin/der Lehrer hat uns gesagt, dass wir uns am Unterrichts- } \\
\text { gespräch beteiligen können ohne Angst zu haben etwas Falsches zu } \\
\text { sagen. }\end{array}$ \\
\hline Aktrv2 & $\begin{array}{l}\text { Die Lehrerin/der Lehrer hat uns immer wieder ermutigt, uns am Un- } \\
\text { terrichtsgespräch zu beteiligen, wenn es nötig war. }\end{array}$ \\
\hline Aktrv3 & $\begin{array}{l}\text { Die Lehrerin/der Lehrer hat uns daran erinnert, dass wir uns gegen- } \\
\text { seitig zuhören sollen, wenn es nötig war. }\end{array}$ \\
\hline
\end{tabular}

Skala Lernbegleitung - Zielorientierung SK_lbzo

Quelle: Eigenentwicklung

Ausprägung: $\quad 3$ trifft zu

2 trifft eher $z u$

1 trifft eher nicht zu

0 trifft nicht $z u$

Anweisung: Wenn du an das heutige Unterrichtsgespräch denkst, wie hat der/die Lehrer/in mit euch interagiert?

\begin{tabular}{|l|l|l|l|l|}
\hline Skala & ALPHA $_{\text {MZP01 }}$ & ALPHA $_{\text {MZP02 }}$ & ALPHA $_{\text {MZP04 }}$ & ALPHA $_{\text {MZP05 }}$ \\
\hline SK_Ibzo & .60 & .68 & .70 & .80 \\
\hline
\end{tabular}

\begin{tabular}{|l|l|}
\hline VARIABLE & Item - Wortlaut \\
\hline Lbzo1 & $\begin{array}{l}\text { Die Lehrerin/der Lehrer hat uns klar gemacht, was wir in der heutigen } \\
\text { Unterrichtsstunde lernen sollen. }\end{array}$ \\
\hline Lbzo2 & $\begin{array}{l}\text { Die Lehrerin/der Lehrer hat uns klar gemacht, was wir machen müs- } \\
\text { sen, um das Lernziel zu erreichen. }\end{array}$ \\
\hline Lbzo3 & $\begin{array}{l}\text { Die Lehrerin/der Lehrer hat uns klar gemacht, was von uns erwartet } \\
\text { wird. }\end{array}$ \\
\hline
\end{tabular}


84 Dialogische Gesprächsführung im Unterricht

Skala Empfinden im Unterrichtsgespräch SK_empf

Quelle: Eigenentwicklung

Ausprägung: $\quad 3$ trifft $z u$

2 trifft eher $z u$

1 trifft eher nicht zu

0 trifft nicht zu

Anweisung: Ich fühle mich wohl,...

\begin{tabular}{|l|l|l|l|l|}
\hline Skala & ALPHAMZP01 & ALPHAMZP02 & ALPHAMZP04 & ALPHAMZP05 \\
\hline SK_empf & .64 & .69 & .71 & .67 \\
\hline
\end{tabular}

\begin{tabular}{|l|l|}
\hline VARIABLE & Item - Wortlaut \\
\hline Empf1 & ..wenn ich in einer Antwort eigene Ideen äußern kann. \\
\hline Empf2 & $\ldots$ wenn meine Mitschüler mir zuhören. \\
\hline Empf3 & $\begin{array}{l}\text {..wenn meine Antworten relevant für den weiteren Verlauf der Stun- } \\
\text { de sind. }\end{array}$ \\
\hline
\end{tabular}




\section{Publikationen im Projekt Dialogue}

Weitere Informationen und Analysen der im Rahmen der Dialogue-Studien (Dialogue I \& II) entstandenen Daten finden sich in den nachfolgend, alphabetisch aufgeführten bisherigen Publikationen:

Alles, M., Seidel, T. \& Gröschner, A. (2019). Establishing a positive learning atmosphere and conversation culture in the context of a video-based teacher learning community. Professional Development in Education, 45(2), 250-263. https://doi.org/10.1080/194152 57.2018.1430049.

Alles, M., Seidel, T. \& Gröschner, A. (2018). Toward better goal clarity in instruction: How focus on content, social exchange and active learning supports teachers in improving dialogic teaching practices. International Education Studies (IES), 11(1), 11-24. Retrieve online: http://www.ccsenet.org/journal/index.php/ies/article/view/69972/39758

Chang, Ch.-F., Gröschner, A., Hall, N.C., Alles, M., Seidel, T. (2018). Exploring Teachers' Emotions Via Nonverbal Behavior during Video-based Teacher Professional Development. AERA Open, 4(4), 1-15. https://doi.org/10.1177/2332858418819851.

Gröschner, A., Schindler, A.-K., Holzberger, D., Alles, M. \& Seidel, T. (2018). How systematic video reflection in teacher professional development regarding classroom discourse contributes to teacher and student self-efficacy. International Journal of Educational Research, 90, 223-233. https://doi.org/10.1016/j.ijer.2018.02.003.

Gröschner, A., Seidel, T., Kiemer, K. \& Pehmer, A.-K. (2015). Through the lens of teacher professional development components: the "Dialogic Video Cycle" as an innovative program to foster classroom dialogue. Professional Development in Education, 41(4), 729-756. https://doi.org/10.1080/19415257.2014.939692.

Gröschner, A., Seidel, T., Pehmer, A.-K. \& Kiemer, K. (2014). Facilitating collaborative teacher learning: the role of "mindfulness" in video-based teacher professional development programs. Gruppendynamik und Organisationsberatung, 45(3), 273-290. https:// doi.org/10.1007/s11612-014-0248-0.

Kiemer, K., Gröschner, A., Kunter, M. \& Seidel, T. (2018). Instructional and motivational classroom discourse and their relationship with teacher autonomy and competence support - findings from teacher professional development. European Journal of Psychology of Education, 33(2), 377-402. http://dx.doi.org/10.1007/s10212-016-0324-7.

Kiemer, K., Gröschner, A., Pehmer, A.-K. \& Seidel, T. (2015). Effects of a classroom discourse intervention on teachers' practice and students' motivation to learn mathematics and science. Learning and Instruction, 35(2), 94-103. http://dx.doi.org/10.1016/j.learnins truc.2014.10.003.

Kiemer, K., Gröschner, A., Pehmer, A.-K. \& Seidel, T. (2014). Teacher learning and student outcomes in the context of classroom discourse - Findings from a video-based teacher professional development programme. Form@re, 2, 51-62. http://dx.doi.org/10.13128/ formare-15124.

Pehmer, A.-K., Gröschner, A. \& Seidel, T. (2015). Fostering and scaffolding student engagement in productive classroom discourse: Teachers' practice changes and reflections in light of teacher professional development. Learning, Culture and Social Interaction, 7, 12-27. https://doi.org/10.1016/j.lcsi.2015.05.001.

Pehmer, A.-K., Gröschner, A. \& Seidel, T. (2015). How teacher professional development regarding classroom dialogue affects students' higher order learning. Teaching and Teacher Education, 47(2), 108-119. https://doi.org/10.1016/j.tate.2014.12.007.

Pielmeier, M., Böheim, R., Schindler, A.-K., Gröschner, A., Knogler, M., Jerabek, M. \& Seidel, T. (2018). Fostering Dialogic Teaching - A video-based TPD program to improve classroom discourse. In K. Smith (Ed.), NAFOL Year book 2017 (pp. 63-87). Norway: Fagbokforlaget Vigmostad \& Bjorke AS.

Schindler, A.-K., Gröschner, A. \& Seidel, T. (2015). Teaching science in different ways: A comparative case study on student verbal engagement in classroom dialogue. Orbis Scholae, 9(2), 9-34. https://doi.org/10.14712/23363177.2015.78.

Weil, M., Seidel, T., Schindler, A.-K., Gröschner, A. (2020). Opening 'windows' for teachers to change classroom discourse. Learning, Culture and Social Interaction, 26. https://doi. org/10.1016/j.lcsi.2020.100425 


\section{Literaturverzeichnis}

Alexander, R. (2018). Towards dialogic teaching: Rethinking classroom talk (5. edition). Thirsk: Dialogos.

Alexander, R. (2005). Towards dialogic teaching: Rethinking classroom talk (2nd edition). Cambridge: Dialogos.

Alexander, R. (2018). Developing dialogic teaching: Genesis, process, trial. Research Papers in Education, 33(5), 561-598. https://doi.org/10.1080/02671522.2018.1481140

Alles, M., Seidel, T., \& Gröschner, A. (2018). Establishing a positive learning atmosphere and conversation culture in the context of a video-based teacher learning community. Professional Development in Education, 11(1), 1-14. https://doi.org/10.1080/194152 57.2018.1430049

Bakeman, R., \& Gottman, J. M. (1997). Observing Interaction: An introduction to sequential analysis. Cambridge University Press. https://doi.org/10.1017/CBO9780511527685

Begrich, L., Fauth, B., Kunter, M., \& Klieme, E. (2017). Wie informativ ist der erste Eindruck? Das Thin-Slices-Verfahren zur videobasierten Erfassung des Unterrichts. Zeitschrift für Erziehungswissenschaft.

Borko, H., Jacobs, J., Eiteljorg, E. \& Pittman, M. E. (2008). Video as a Tool for Fostering Productive Discussions in Mathematics Professional Development. Teaching and Teacher Education, 24, 417-436. https://doi.org/10.1016/j.tate.2006.11.012

Borko, H., Jacobs, J., \& Koellner, K. (2010). Contemporary Approaches to Teacher Professional Development. In P. Peterson (Ed.), International encyclopedia of education (3rd ed., pp. 548-556). Oxford: Elsevier. https://doi.org/10.1016/B978-0-08-0448947.00654-0

Chi, M. T. H. (2009). Active-constructive-interactive: A conceptual framework for differentiating learning activities. Topics in Cognitive Science, 1(1), 73-105. https://doi. org/10.1111/j.1756-8765.2008.01005.x

Clarke, D., \& Hollingsworth, H. (2002). Elaborating a model of teacher professional growth. Teaching and Teacher Education, 18(8), 947-967. https://doi.org/10.1016/S0742051X(02)00053-7

Clausen, M., Reusser, K., \& Klieme, E. (2003). Unterrichtsqualität auf der Basis hoch-inferenter Unterrichtsbeurteilungen. Ein Vergleich zwischen Deutschland und der deutschsprachigen Schweiz. Unterrichtswissenschaft, 31(2), 122-141.

Darling-Hammond, L., Hyler, M. E., \& Gardner, M. (2017). Effective Teacher Professional Development. Palo Alto/CA: Learning Policy Institute.

Desimone, L. (2009). Improving Impact Studies of Teachers' Professional Development: Toward Better Conceptualizations and Measures. Educational Researcher, 38(3), 181199. https://doi.org/10.3102/0013189X08331140

Gee, J. P., \& Green, J. L. (1998). Discourse analysis, learning, and social practice: A methodological study. Review of Research in Education, 23, 119-169. Retrieved from https:// www.scopus.com/inward/record.uri?eid=2-s2.0-0039065314\&partnerID=40\&md5=01d 5b13b91578cd31381d88501ae20e6

Gillies, R. M. (2014). Developments in classroom-based talk. International Journal of Educational Research, 63, 63-68. https://doi.org/10.1016/j.ijer.2013.05.002

Gomez Zaccarelli, F., Schindler, A.-K., Borko, H., \& Osborne, J. (2018). Learning from professional development: A case study of the challenges of enacting productive science discourse in the classroom. Professional Development in Education, 9(2), 1-17. https:// doi.org/10.1080/19415257.2017.1423368

Gröschner, A., Schindler, A.-K., Holzberger, D., Alles, M., \& Seidel, T. (2018). How systematic video reflection in teacher professional development regarding classroom discourse contributes to teacher and student self-efficacy. International Journal of Educational Research. Advance online publication. https://doi.org/10.1016/j.ijer.2018.02.003

Gröschner, A., Seidel, T., Kiemer, K., \& Pehmer, A.-K. (2015). Through the lens of teacher professional development components: The 'Dialogic Video Cycle' as an innovative program to foster classroom dialogue. Professional Development in Education, 41(4), 729-756. https://doi.org/10.1080/19415257.2014.939692

Gröschner, A., Seidel, T., Pehmer, A.-K., \& Kiemer, K. (2014). Facilitating collaborative teacher learning: The role of "mindfulness" in video-based teacher professional development programs. Gruppendynamik und Organisationsberatung, 45(3), 273-290. https://doi.org/10.1007/s11612-014-0248-0 
Hattie, J., \& Timperley, H. (2007). The Power of Feedback. Review of Educational Research, 77(1), 81-112. https://doi.org/10.3102/003465430298487

Häusler, J., Jurik, V., Schindler, A.-K., Gröschner, A., \& Seidel, T. (2019). Videografie im Unterricht. In M. Harring, C. Rohlfs, \& M. Gläser-Zikuda (Eds.), UTB Schulpädagogik: Vol. 8698. Handbuch Schulpädagogik (pp. 831-840). Münster, New York: Waxmann.

Hennessy, S., Rojas-Drummond, S., Higham, R., Márquez, A. M., Maine, F., Ríos, R. M., García-Carrión, R., Torreblanca, O. \& Barrera, M. J. (2016). Developing a coding scheme for analysing classroom dialogue across educational contexts. Learning, Culture and Social Interaction, 9, 16-44. https://doi.org/10.1016/j.lcsi.2015.12.001

Higham, R. J. E., Brindley, S., \& van de Pol, J. (2014). Shifting the primary focus: Assessing the case for dialogic education in secondary classrooms. Language and Education, 28(1), 86-99. https://doi.org/10.1080/09500782.2013.771655

Hofmann, R., \& Ruthven, K. (2018). Operational, interpersonal, discussional and ideational dimensions of classroom norms for dialogic practice in school mathematics. British Educational Research Journal, 44(3), 496-514. https://doi.org/10.1002/berj.3444

Howe, C., \& Abedin, M. (2013). Classroom dialogue: A systematic review across four decades of research. Cambridge Journal of Education, 43(3), 325-356. https://doi.org/10.1 080/0305764X.2013.786024

Hugener, I. (2006). Sozialformen und Lektionsdauer. In E. Klieme, K. Rakoczy, A. Buff, F. Lipowsky, I. Hugener, C. Pauli, \& K. Reusser (Eds.), Materialien zur Bildungsforschung: Vol. 15. Dokumentation der Erhebungs- und Auswertungsinstrumente zur schweizerisch-deutschen Videostudie „Unterrichtsqualität, Lernverhalten und mathematisches Verständnis“ (pp. 55-61). Frankfurt am Main: Gesellschaft zur Förderung Pädagogischer Forschung.

Igler, J., Ohle-Peters, A., \& McElvany, N. (Februar, 2018). Reihenfolgeeffekte bei Videoratings von Unterrichtsqualität. 6. Tagung der Gesellschaft für Empirische Bildungsforschung (GEBF), Basel (Schweiz).

Jurik, V., Gröschner, A., \& Seidel, T. (2013). How student characteristics affect girls' and boys' verbal engagement in physics instruction. Learning and Instruction, 23, 33-42. https:// doi.org/10.1016/j.learninstruc.2012.09.002

Kennedy, M. M. (2016). How Does Professional Development Improve Teaching? Review of Educational Research, 86(4), 945-980. https://doi.org/10.3102/0034654315626800

Kiemer, K., Gröschner, A., Pehmer, A.-K., \& Seidel, T. (2014). Teacher learning and student outcomes in the context of classroom discourse. Findings from a video-based teacher professional development programme. Form@re, 2, 51-62. https://doi.org/10.13128/formare-15124

Knogler, M., Harackiewicz, J. M., Gegenfurtner, A., \& Lewalter, D. (2015). How situational is situational interest?: Investigating the longitudinal structure of situational interest. Contemporary Educational Psychology, 43, 39-50. https://doi.org/10.1016/j.ced psych.2015.08.004

Kunter, M., Baumert, J., Leutner, D., Terhart, E., Seidel, T., Dicke, T., Holzberger, D., Kunina-Habenicht, O., Linninger, C., Lohse-Bossenz, H., Schulze-Stocker \& F. Stürmer, K. (2017). Dokumentation der Erhebungsinstrumente der Projektphasen des BilWiss-Forschungsprogramms von 2009 bis 2016: Bildungswissenschaftliches Wissen und der Erwerb professioneller Kompetenz in der Lehramtsausbildung (BilWiss); die Bedeutung des bildungswissenschaftlichen Hochschulwissens für den Berufseinstieg von Lehrkräften (BilWiss-Beruf). Frankfurt am Main, Frankfurt am Main: GoetheUniversität; Universitätsbibliothek Johann Christian Senckenberg. https://doi.org/10. 1007/978-3-658-07274-2_3

Kunter, M., Kunina-Habenicht, O., Baumert, J., Dicke, T., Holzberger, D., Lohse-Bossenz, ... Terhart, E. (2017). Bildungswissenschaftliches Wissen und professionelle Kompetenz in der Lehramtsausbildung. Ergebnisse des Projekts BilWiss. In C. Gräsel \& K. Trempler (Eds.), Entwicklung von Professionalität pädagogischen Personals. Wiesbaden: Springer Fachmedien.

Kunter, M., \& Trautwein, U. (2013). Psychologie des Unterrichts. StandardWissen Lehramt: Vol. 3895. Paderborn: Schöningh. Retrieved from http://www.utb-studi-e-book.de/9783 838538952

Lipowsky, F., Rakoczy, K., Pauli, C., Drollinger-Vetter, B., Klieme, E., \& Reusser, K. (2009). Quality of geometry instruction and its short-term impact on students' understanding of the Pythagorean Theorem. Learning and Instruction, 19(6), 527-537. https://doi. org/10.1016/j.learninstruc.2008.11.001

Mangold. (2017). INTERACT: Mangold International GmbH. 
Marsh, H. W., Trautwein, U., Ludtke, O., Koller, O., \& Baumert, J. (2005). Academic selfconcept, interest, grades, and standardized test scores: Reciprocal effects models of causal ordering. Child Development, 76(2), 397-416. https://doi.org/10.1111/j.14678624.2005.00853.x

Mehan, H. (1979). Learning lessons. Cambrigde, MA: Harvard University Press. https://doi. org/10.4159/harvard.9780674420106

Mercer, N., \& Dawes, L. (2014). The study of talk between teachers and students, from the 1970s until the 2010s. Oxford Review of Education, 40(4), 430-445. https://doi.org/10. 1080/03054985.2014.934087

Mercer, N., \& Littleton, K. (2007). Dialogue and the development of children's thinking: A sociocultural approach (1. publ). London u.a.: Routledge. https://doi.org/10.4324/ 9780203946657

Michaels, S., \& O’Connor, C. (2012). Talk Science Primer. Cambrigde, MA: TERC. Retrieved from http://www.cgcs.org/cms/lib/DC00001581/Centricity/Domain/155/TalkScience_ Primer.pdf

Michaels, S., O’Connor, C., \& Resnick, L. B. (2008). Deliberative Discourse Idealized and Realized: Accountable Talk in the Classroom and in Civic Life. Studies in Philosophy and Education, 27(4), 283-297. https://doi.org/10.1007/s11217-007-9071-1

Michaels, S., \& O’Connor, C. (2015). Conceptualizing talk moves as tools: Professional development approaches for academically productive discussions. In L. B. Resnick, C. S.C. Asterhan, \& S. N. Clarke (Eds.), Socializing intelligence through academic talk and dialogue (pp. 333-347). Washington, DC: American Educational Research Association.

Mortimer, E. F., \& Scott, P. (2010). Meaning making in secondary science classrooms. Buckingham: Open University Press. Retrieved from http://search.ebscohost.com/login. aspx ?direct $=$ true $\&$ scope $=$ site $\& \mathrm{db}=$ nlebk $\& \mathrm{db}=$ nlabk\&AN $=234040$

O'Connor, C., Michaels, S., Chapin, S., \& Harbaugh, A. G. (2017). The silent and the vocal: Participation and learning in whole-class discussion. Learning and Instruction, 48, 5-13. https://doi.org/10.1016/j.learninstruc.2016.11.003

Osborne, J., Borko, H., Bush, K. C., Fishman, E., Million, S., \& Tseng, A. (2016). Developing and Using an Instrument to assess the dialectical potential of whole - class discussions in teaching science, Washington, DC.

Osborne, J., Borko, H., Fishman, E., Gomez Zaccarelli, F., Berson, E., Busch, K. C., Reigh, E. \& Tseng, A. (2019). Impacts of a Practice-Based Professional Development Program on Elementary Teachers' Facilitation of and Student Engagement With Scientific Argumentation. American Educational Research Journal. Advance online publication. https://doi.org/10.3102/0002831218812059

Osborne, J., Simon, S., Christodoulou, A., Howell-Richardson, C., \& Richardson, K. (2013). Learning to argue: A study of four schools and their attempt to develop the use of argumentation as a common instructional practice and its impact on students. Journal of Research in Science Teaching, 50(3), 315-347. https://doi.org/10.1002/tea.21073

Pauli, C. (2014). Ratingverfahren. Journal für LehrerInnenbildung, 14(1), 56-59.

Pehmer, A.-K., Gröschner, A., \& Seidel, T. (2015a). Fostering and scaffolding student engagement in productive classroom discourse: Teachers' practice changes and reflections in light of teacher professional development. Learning, Culture and Social Interaction, 7, 12-27. https://doi.org/10.1016/j.lcsi.2015.05.001

Pehmer, A.-K., Gröschner, A., \& Seidel, T. (2015b). How teacher professional development regarding classroom dialogue affects students' higher-order learning. Teaching and Teacher Education, 47, 108-119. https://doi.org/10.1016/j.tate.2014.12.007

Pielmeier, M., Böheim, R., Schindler, A.-K., Gröschner, A., Knogler, M., Alles, M., \& Seidel, T. (2018). Fostering Dialogic Teaching - The "Dialogic Video Cycle" as a video based professional development programme to enhance classroom discourse. In K. Smith (Ed.), Norsk OG Internasjonal Lorerutdanningsforskning (pp. 63-88). Bergen: Fagbokforl.

Rakoczy, K., Buff, A., \& Klieme, E. (2005). Dokumentation der Erhebungs- und Auswertungsinstrumente zur schweizerisch-deutschen Videostudie. „Unterrichtsqualität, Lernverhalten und mathematisches Verständnis“. 1. Befragungsinstrumente. Materialien zur Bildungsforschung: Vol. 13. Frankfurt am Main: GFPF. Retrieved from http://nbn-resolv ing.de/urn:nbn:de:0111-opus-31060

Rakoczy, K., \& Pauli, C. (2006). Hoch-inferentes Rating: Beurteilung der Qualität unterrichtlicher Prozesse. In E. Klieme, K. Rakoczy, A. Buff, F. Lipowsky, I. Hugener, C. Pauli, \& K. Reusser (Eds.), Materialien zur Bildungsforschung: Vol. 15. Dokumentation der Erhebungs- und Auswertungsinstrumente zur schweizerisch-deutschen Video- 
studie „Unterrichtsqualität, Lernverhalten und mathematisches Verständnis“ (pp. 206233). Frankfurt am Main: Gesellschaft zur Förderung Pädagogischer Forschung.

Ramm, G. C., Prenzel, M., Baumert, J., Blum, W., Lehmann, R., Leutner, D., Neubrand, M., Pekrun, R., Rolff, H.-G., Rost, J. \& Schiefele, U. (Eds.) (2006). PISA 2003: Dokumentation der Erhebungsinstrumente. Münster: Waxmann.

Resnick, L. B., Asterhan, C. S. C., \& Clarke, S. N. (Eds.). (2015). Socializing intelligence through academic talk and dialogue. Washington, DC: American Educational Research Association. https://doi.org/10.3102/978-0-935302-43-1

Resnick, L. B., Michaels, S., \& O'Connor, C. (2010). How (well-structured) talk builds the mind. In D. Preiss \& R. J. Sternberg (Eds.), Innovations in educational psychology: Perspectives on learning, teaching, and human development (pp. 163-194). New York: Springer Pub.

Reznitskaya, A., Wilkinson, I. A. G., \& Oyler, J. (2016). The Argumentation Rating Tool.

Reznitskaya, A., Wilkinson, I. A. G., Oyler, J., Bourdage-Reninger, K., \& Sykes, A. (2016, April). Using the Argumentation Rating Tool to Support Teacher Facilitation of Inquiry Dialogue in Elementary Language Arts Classrooms. Paper presented at the Annual Meeting of the American, Washington, DC.

Richter, D., Kunter, M., Lüdtke, O., Klusmann, U., \& Baumert, J. (2011). Soziale Unterstützung beim Berufseinstieg ins Lehramt. Zeitschrift für Erziehungswissenschaft, 14(1), 35-59. https://doi.org/10.1007/s11618-011-0173-8

Rzejak, D., Künsting, J., Lipowsky, F., Fischer, E., Dezhgahi, U., \& Reichardt, A. (2014). Facetten der Lehrerfortbildungsmotivation - eine faktorenanalytische Betrachtung. Journal for Educational Research Online, 6(1), 139-159.

Schwarzer, R., \& Schmitz, G. S. (1999). Skala zur Lehrer-Selbstwirksamkeitserwartung (WIRKLEHR). In R. Schwarzer \& M. Jerusalem (Eds.), Skalen zur Erfassung von Lehrer- und Schülermerkmalen: Dokumentation der psychometrischen Verfahren im Rahmen der wissenschaftlichen Begleitung des Modellversuchs Selbstwirksame Schulen (pp. 60-61). Berlin: R. Schwarzer.

Sedova, K., Sedlacek, M., Svaricek, R., Majcik, M., Navratilova, J., Drexlerova, A., Kychler, J. \& Salamounova, Z. (2019). Do those who talk more learn more?: The relationship between student classroom talk and student achievement. Learning and Instruction, 63, 101217. https://doi.org/10.1016/j.learninstruc.2019.101217

Seidel, T., \& Shavelson, R. J. (2007). Teaching Effectiveness Research in the Past Decade: The Role of Theory and Research Design in Disentangling Meta-Analysis Results. Review of Educational Research, 77(4), 454-499. https://doi.org/10.3102/ 0034654307310317

Seidel, T. (2014). Lehrerhandeln im Unterricht [Teacher Behavior in classroom]. In E. Terhart, H. Bennewitz, \& M. Rothland (Eds.), Handbuch der Forschung zum Lehrerberuf (2nd ed., pp. 781-806). Münster, New York: Waxmann.

Seidel, T., Dalehefte, I. M., Lehrke, M., \& Trepke, C. (2005). Kapitel 13 Lehrerkurzfragebogen. In T. Seidel, M. Prenzel, \& M. Kobarg (Eds.), How to run a video study: Technical report of the IPN Video Study (pp. 309-316). Münster: Waxmann.

Seidel, T., \& Prenzel, M. (2006). Stability of teaching patterns in physics instruction: Findings from a video study. Learning and Instruction, 16(3), 228-240. https://doi.org/10.1016/j. learninstruc.2006.03.002

Seidel, T., Prenzel, M., Duit, R., \& Lehrke, M. (Eds.). (2003). IPN-Materialien. Technischer Bericht zur Videostudie „Lehr-Lern-Prozesse im Physikunterricht“. Kiel: IPN. Retrieved from http://archiv.ipn.uni-kiel.de/buecherarchiv/buch_videostudie2.html

Seidel, T., Prenzel, M., \& Kobarg, M. (Eds.). (2005). How to run a video study: Technical report of the IPN Video Study. Münster: Waxmann.

Seidel, T., Prenzel, M., Rimmele, R., Dalehefte, I. M., Herweg, C., Kobarg, M., \& Schwindt, K. (2006). Blicke auf den Physikunterricht. Ergebnisse der IPN Videostudie. Zeitschrift für Pädagogik, 52(6), 799-821.

Seidel, T., \& Reiss, K. (2014). Lerngelegenheiten im Unterricht. In T. Seidel \& A. Krapp (Eds.), Psychologie 2014. Pädagogische Psychologie (6th ed., pp. 253-275). Weinheim: Julius Beltz.

Sinclair, J., \& Coulthard, R. M. (1975). Towards an analysis of discourse: The English used by teachers and pupils. London: Oxford Univ. Press.

Sprott, R. A. (2019). Factors that foster and deter advanced teachers' professional development. Teaching and Teacher Education, 77, 321-331. https://doi.org/10.1016/j.tate. 2018.11.001 
Van den Bergh, L., Ros, A., \& Beijaard, D. (2015). Teacher learning in the context of a continuing professional development programme: A case study. Teaching and Teacher Education, 47, 142-150. https://doi.org/10.1016/j.tate.2015.01.002

Vescio, V., Ross, D., \& Adams, A. (2008). A review of research on the impact of professional learning communities on teaching practice and student learning. Teaching and Teacher Education, 24(1), 80-91. https://doi.org/10.1016/j.tate.2007.01.004

Walshaw, M., \& Anthony, G. (2008). The Teacher's Role in Classroom Discourse: A Review of Recent Research Into Mathematics Classrooms. Review of Educational Research, 78(3), 516-551. https://doi.org/10.3102/0034654308320292

Wilson, S. M. (2013). Professional development for science teachers. Science (New York, N.Y.), 340(6130), 310-313. https://doi.org/10.1126/science.1230725

Wirtz, M. A., \& Caspar, F. (2002). Beurteilerübereinstimmung und Beurteilerreliabilität: Methoden zur Bestimmung und Verbesserung der Zuverlässigkeit von Einschätzungen mittels Kategoriensystemen und Ratingskalen. Göttingen: Hogrefe Verl. für Psychologie.

Zimmermann, M., Moser, M., Wischgoll, A., Reusser, K., \& Pauli, C. (2019, February). Wie verändern sich Überzeugungen und Handeln von Lehrpesonen während einer Weiterbildung? Fallanalysen einer Interventionsstudie zu dialogischer Gesprächsführung im Geschichtsunterricht. Gesellschaft für Empirische Bildungsforschung. Symposium „Unterrichtsgespräche ,dialogisch` führen: Entwicklung einer Kernkompetenz von (angehenden) Lehrpersonen“, Köln. 